Klinik für Kinderkardiologie und angeborene Herzfehler Deutsches Herzzentrum München des Freistaates Bayern Klinik an der Technischen Universität München (Direktor: Prof. Dr. Peter Ewert)

\title{
Auswirkungen einer frühzeitigen partiellen cavopulmonalen Anastomose auf das Wachstum der Pulmonalarterien
}

Antonia Monika Dorothee Presch

Vollständiger Abdruck der von der Fakultät für Medizin der Technischen Universität München zur Erlangung des akademischen Grades eines Doktors der Medizin genehmigten Dissertation.

Vorsitzender:

Prüfer der Dissertation:
Prof. Dr. Jürgen Schlegel

1. Prof. Dr. Peter Ewert

2. Prof. Dr. Renate M. Oberhoffer

Die Dissertation wurde am 26.03.2018 bei der Technischen Universität München eingereicht und durch die Fakultät für Medizin am 09.10.2018 angenommen. 


\section{Inhaltsverzeichnis}

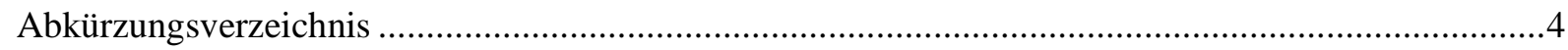

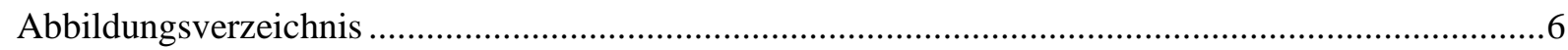

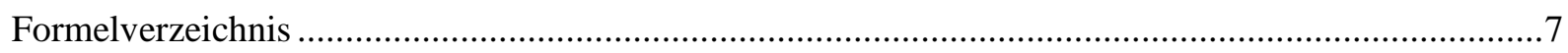

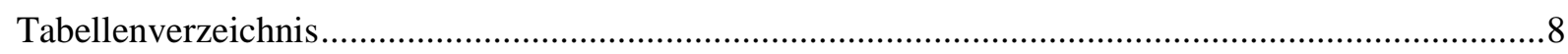

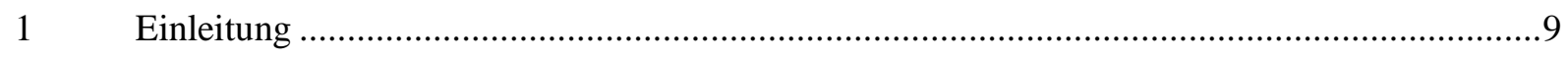

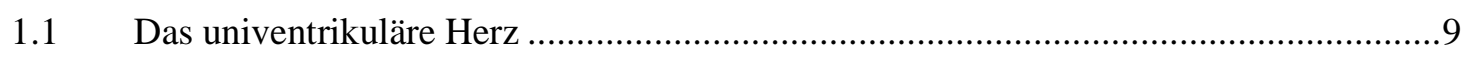

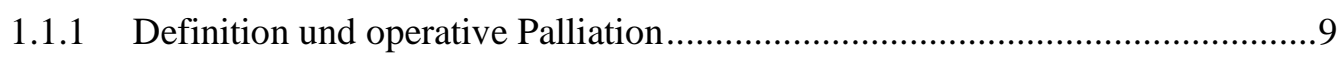

1.1.2 Eigenschaften der verschiedenen Shuntarten............................................. 10

1.1.3 Voraussetzungen für die Durchführung der partiellen cavopulmonalen

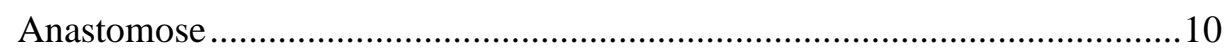

1.2 Der Zeitpunkt der partiellen cavopulmonalen Anastomose........................................11

1.2.1 Bedeutung einer frühzeitigen Durchführung der partiellen cavopulmonalen Anastomose im klinischen Kontext ...........................................................11

1.2.2 Der Einfluss der partiellen cavopulmonalen Anastomose auf das Wachstum

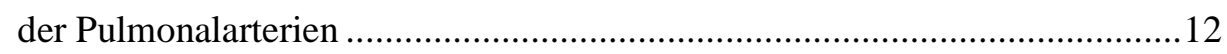

1.2.3 Möglicher Einfluss des Alters zum Zeitpunkt der partiellen cavopulmonalen Anastomose auf das Wachstum der Pulmonalarterien - Ziel der Arbeit.........12

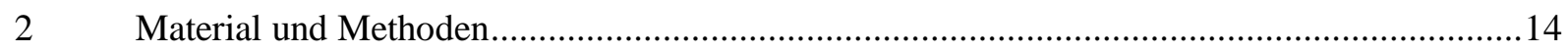

2.1 Patientenkollektiv: Rekrutierung und Gruppenbildung ............................................... 14

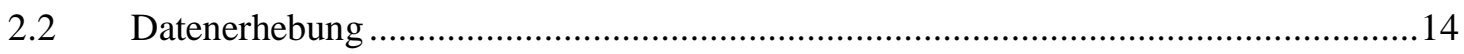

2.2.1 Erhebung der deskriptiven Daten .................................................................. 14

2.2.2 Erhebung der hämodynamischen Daten .........................................................15

2.2.3 Vermessung der Pulmonalarterien und Berechnung der Indices ..................... 15

2.2.4 Erhebung der perioperativen und postoperativen Daten ...............................17

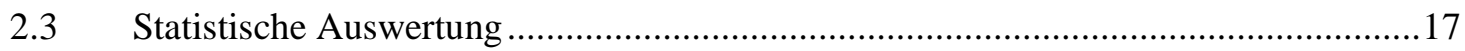

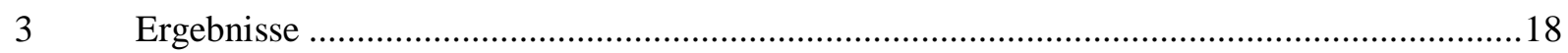

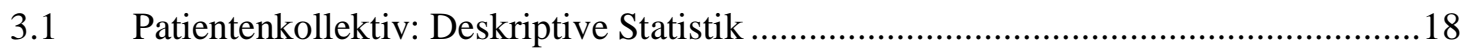

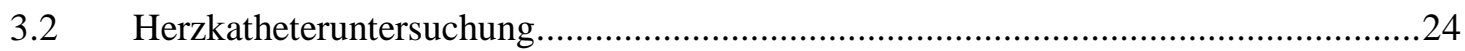

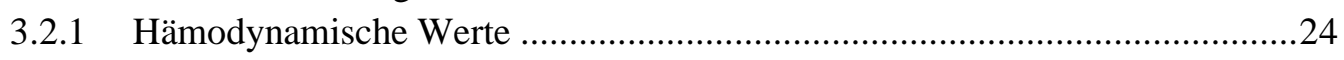

3.2.2 Größe und Wachstum der Pulmonalarterien..............................................25

3.2.2.1 Rechte Pulmonalarterie ...............................................................25

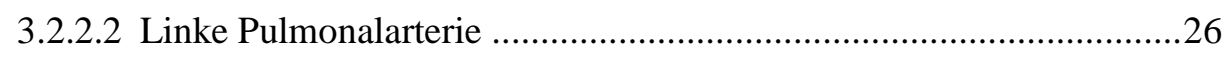

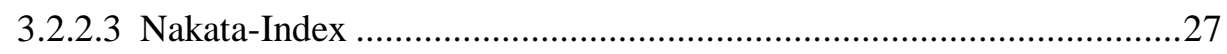

3.2.2.4 Vergleich der Pulmonalarterien zwischen Patienten mit systemicopulmonalen Shunt und Patienten mit right-ventricle-to-pulmonary-

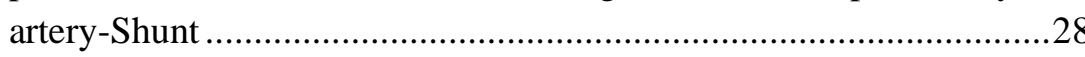


3.2.2.5 Vergleich der Pulmonalarterien zwischen Patienten mit hypoplastischem Linksherzsyndrom und Patienten mit anderen Herzfehlern

3.2.2.6 Einflussfaktoren auf die Größe der Pulmonalarterien

3.3 Perioperativer Verlauf: Dauer des Kreislaufstillstandes mittels

Herz-Lungen-Maschine

3.4 Postoperativer Verlauf nach Durchführung der partiellen cavopulmonalen

Anastomose

3.4.1 Beatmungsdauer, arterielle Sauerstoffsättigung, Sauerstoffbedarf und transkutane Sauerstoffsättigung bei Entlassung ............................................37

3.4.2 Pulmonalarterieller Druck und transpulmonaler Gradient .............................39

3.4.3 Dauer des intensivmedizinischen und stationären Aufenthaltes .....................40

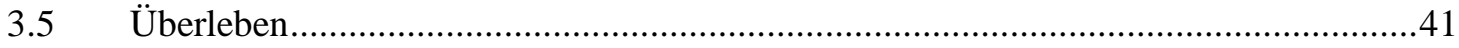

3.5.1 Postoperative Mortalität und Gesamtmortalität ...............................................4 41

3.5.2 Einflussfaktoren auf die Mortalität ..............................................................4

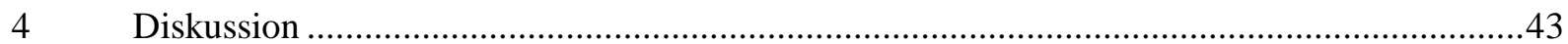

4.1 Größe und Wachstum der Pulmonalarterien ...............................................................43

4.2 Postoperativer Verlauf, Mortalität und Komplikationen ............................................45

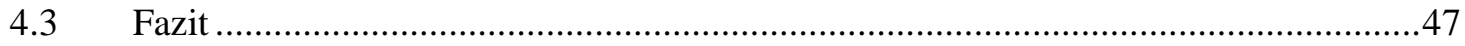

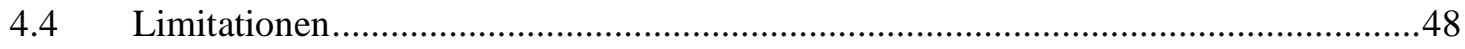

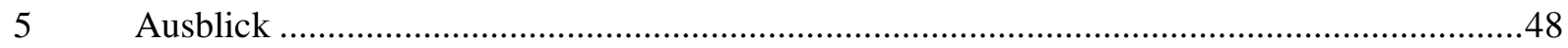

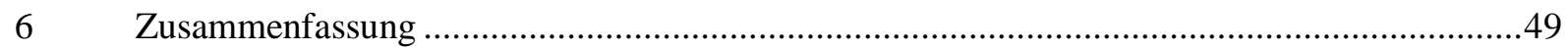

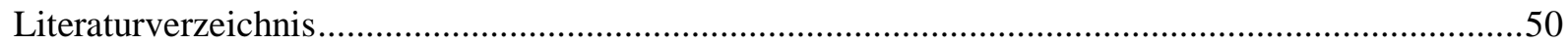

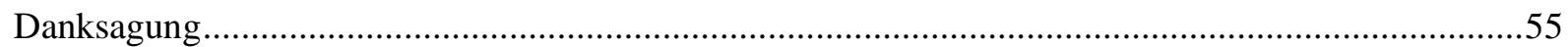




\section{Abkürzungsverzeichnis}

$\begin{array}{ll}\text { bzw. } & \text { beziehungsweise } \\ \mathrm{cm} & \text { Centimeter } \\ \mathrm{d} & \text { Tage } \\ \mathrm{g} & \text { Gramm }\end{array}$

ggf. gegebenenfalls

$\mathrm{h} \quad$ Stunden

HLHS hypoplastisches Linksherzsyndrom

$\mathrm{kg} \quad$ Kilogramm

KOF Körperoberfläche

LAP linksatrialer Druck

LPA linke Pulmonalarterie

m Monate

$\mathrm{m}^{2} \quad$ Quadratmeter

mBT-Shunt modifizierter Blalock-Taussig-Shunt

$\min \quad$ Minuten

$\mathrm{mm}^{2} \quad$ Quadratmillimeter

$\mathrm{mmHg} \quad$ Millimeter-Quecksilbersäule

$\mathrm{O}_{2} \quad$ Sauerstoff

PA Pulmonalarterie(n)

PAP pulmonalarterieller Druck

PCPA partielle cavopulmonale Anastomose

p.o. postoperativ

RAP rechtsatrialer Druck

RPA rechte Pulmonalarterie

RVPA-Shunt right-ventricle-to-pulmonary-artery-Shunt

$\mathrm{SaO}_{2} \quad$ arterielle Sauerstoffsättigung

SP-Shunt systemico-pulmonaler Shunt 
TCPA

$\mathrm{tcSO}_{2}$

TPG

vs.

VEDP

zAP-Shunt

z.B.

ZVD

ZVK totale cavopulmonale Anastomose

transkutane Sauerstoffsättigung

transpulmonaler Gradient

versus

enddiastolischer Ventrikeldruck

zentraler aortopulmonaler Shunt

zum Beispiel

zentraler Venendruck

zentraler Venenkatheter 


\section{Abbildungsverzeichnis}

Abbildung 1: Schematische Darstellung der Messung der Pulmonalarterien .......................................16

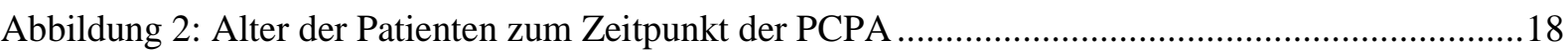

Abbildung 3: Verteilung der verschiedenen Shuntarten in den drei Altersgruppen ............................. 19

Abbildung 4: Vergleich der Körpergröße vor PCPA zwischen den drei Altersgruppen .......................20

Abbildung 5: Vergleich des Körpergewichts vor PCPA zwischen den drei Altersgruppen ...................21

Abbildung 6: Vergleich der Körperoberfläche vor PCPA zwischen den drei Altersgruppen ...............21

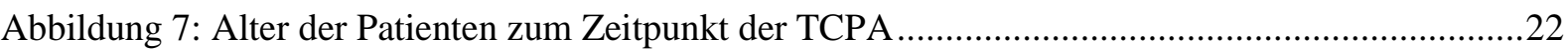

Abbildung 8: Vergleich der Körpergröße vor TCPA zwischen den drei Altersgruppen ........................22

Abbildung 9: Vergleich des Körpergewichts vor TCPA zwischen den drei Altersgruppen..................23

Abbildung 10: Vergleich der Körperoberfläche vor TCPA zwischen den drei Altersgruppen .............23

Abbildung 11: Vergleich der RPA-Flächen-Indices vor PCPA und vor TCPA zwischen und innerhalb

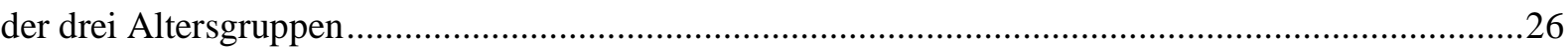

Abbildung 12: Vergleich der LPA-Flächen-Indices vor PCPA und vor TCPA zwischen und innerhalb

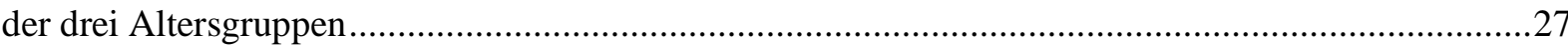

Abbildung 13: Vergleich der Nakata-Indices vor PCPA und vor TCPA zwischen und innerhalb der drei Altersgruppen.

Abbildung 14: Vergleich der LPA-Flächen-Indices vor PCPA zwischen Patienten mit SP-Shunt und

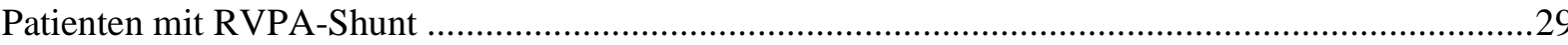

Abbildung 15: Vergleich der Nakata-Indices vor PCPA zwischen Patienten mit SP-Shunt und

Patienten mit RVPA-Shunt

Abbildung 16: Vergleich der RPA-Flächen-Indices vor PCPA zwischen Patienten mit SP-Shunt und

Patienten mit RVPA-Shunt

Abbildung 17: Vergleich der LPA-Flächen-Indices vor TCPA zwischen Patienten mit SP-Shunt und Patienten mit RVPA-Shunt

Abbildung 18: Vergleich der Nakata-Indices vor TCPA zwischen Patienten mit SP-Shunt und

Patienten mit RVPA-Shunt

Abbildung 19: Vergleich der RPA-Flächen-Indices vor TCPA zwischen Patienten mit SP-Shunt und Patienten mit RVPA-Shunt

Abbildung 20: Vergleich der LPA-Flächen-Indices vor PCPA zwischen Patienten mit HLHS und Patienten mit anderen Herzfehlern

Abbildung 21: Vergleich der Nakata-Indices vor PCPA zwischen Patienten mit HLHS und Patienten mit anderen Herzfehlern

Abbildung 22: Vergleich der LPA-Flächen-Indices vor TCPA zwischen Patienten mit HLHS und Patienten mit anderen Herzfehlern

Abbildung 23: Vergleich der Nakata-Indices vor TCPA zwischen Patienten mit HLHS und Patienten mit anderen Herzfehlern

Abbildung 24: Vergleich der postoperativen Beatmungsdauer nach PCPA zwischen den drei

Altersgruppen

Abbildung 25: Vergleich der postoperativen mittleren arteriellen Sauerstoffsättigung nach PCPA

zwischen den drei Altersgruppen

Abbildung 26: Vergleich des 30-Tages-Überlebens nach PCPA zwischen den drei Altersgruppen .....42

Abbildung 27: Vergleich des Gesamtüberlebens nach PCPA zwischen den drei Altersgruppen ...........42 


\section{Formelverzeichnis}

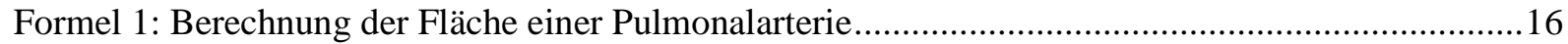

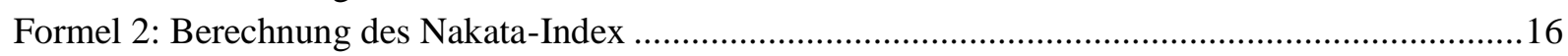

Formel 3: Berechnung des Flächen-Index einer Pulmonalarterie .................................................... 17 


\section{Tabellenverzeichnis}

Tabelle 1: Hämodynamische Parameter vor Durchführung der PCPA im Vergleich zwischen den drei Altersgruppen

Tabelle 2: Hämodynamische Parameter vor Durchführung der TCPA im Vergleich zwischen den drei Altersgruppen.

Tabelle 3: Postoperativer mittlerer pulmonalarterieller Druck und transpulmonaler Gradient im Vergleich zwischen den drei Altersgruppen

Tabelle 4: Mortalität nach Durchführung der PCPA im Vergleich zwischen den drei Altersgruppen.. 41 


\section{Einleitung}

\subsection{Das univentrikuläre Herz}

\subsubsection{Definition und operative Palliation}

Das univentrikuläre Herz ist durch das Vorliegen nur einer voll funktionsfähigen Herzkammer gekennzeichnet, während die zweite Kammer entweder nicht ausgebildet oder nicht funktionsfähig ist. Dies kann sowohl auf den rechten als auch auf den linken Ventrikel zutreffen. Musterbeispiele hierfür sind zum einen die Trikuspidalatresie, bei der die rechte Herzkammer aufgrund einer fehlenden oder atretischen Atrioventrikularklappe keine Auswurfleistung erbringen kann und zum anderen das hypoplastische Linksherzsyndrom (HLHS), bei dem eine Mitral- und/oder Aortenklappenstenose oder -atresie sowie ein unterentwickelter Vorhof und/oder Ventrikel die Funktion der linken Herzkammer nicht ermöglicht (Schmid, Asfour 2009).

Einige weitere angeborene Herzfehler können aufgrund bestimmter anatomischer Besonderheiten ebenfalls nicht biventrikulär - also unter Erhalt beziehungsweise (bzw.) Wiederherstellung der Funktion beider Ventrikel - saniert werden, sodass die Therapie dieser (funktionell) univentrikulären Herzen aus der Palliation nach Fontan und somit der vollständigen Trennung des Systems- und Lungenkreislaufes besteht.

Dies erfolgt durch die Anlage einer partiellen (PCPA) und schließlich einer totalen cavopulmonalen Anastomose (TCPA). Im ersten Operationsschritt wird die obere Hohlvene durch eine End-zu-SeitAnastomose mit der rechten Pulmonalarterie verbunden, sodass das venöse Blut der oberen Körperhälfte passiv in das Gefäßbett beider Lungenhälften fließt (Schmid, Asfour 2009).

Dieses Verfahren wurde in seinen Grundzügen das erste Mal im Jahre 1954 von dem Herzchirurgen William Glenn beschrieben, sodass man trotz Veränderungen im chirurgischen Verfahren von der bidirektionalen Glenn-Anastomose spricht (Glenn et al. 1954).

Die untere Hohlvene wird im Rahmen der TCPA, nach Absetzen vom rechten Vorhof, durch einen intra- oder extrakardialen Tunnel ebenfalls mit der rechten Pulmonalarterie verbunden, sodass nun der gesamte Lungenkreislauf vom Systemkreislauf getrennt ist (Schmid, Asfour 2009).

Diese beiden Operationsschritte erfolgen unter der Voraussetzung, dass zuvor eine adäquate Lungenperfusion stattgefunden hat, welche von der Durchgängigkeit bzw. dem Öffnungsgrad des pulmonalen Ausflusstraktes abhängig ist. Patienten ohne Stenose in diesem Bereich leiden durch die exzessive Lungendurchblutung meist an einer schweren Herzinsuffizienz, die zu einer Volumenüberladung des Ventrikels führt. Diese sollte operativ, zum Beispiel (z.B.) durch ein Banding der Pulmonalarterien, noch vor der PCPA reduziert werden. Auf der anderen Seite kann es durch eine hochgradige Stenose oder Atresie des pulmonalen Ausflusstraktes auch zu einer tiefen Zyanose der Patienten kommen. In diesem Falle ist die Anlage eines Shunts zwischen beiden Kreislaufsystemen oder ein interventionelles Stenting des Ductus arteriosus innerhalb der ersten Lebenstage nötig, um eine adäquate Lungenperfusion gewährleisten zu können. Bei einem obstruktiven systemischen Ausflusstrakt, wie z.B. beim HLHS, ist zusätzlich zur Shuntanlage die Anlage einer Damus-KayeStansel-Anastomose bzw. die Korrektur nach Norwood notwendig, bei der die hypoplastische Aorta ascendens mit dem abgesetzten Pulmonalisstamm verbunden wird und gegebenenfalls (ggf.) der Aortenbogen mittels Patch erweitert wird, um eine adäquate nicht-obstruktive Systemperfusion zu gewährleisten (Schmid, Asfour 2009). 


\subsubsection{Eigenschaften der verschiedenen Shuntarten}

Der Shunt zur Gewährleistung der Lungenperfusion kann auf verschiedene Weisen angebracht werden. Eine erste Unterteilung ist möglich, indem unterschieden wird, ob der Shunt vom Systemventrikel oder von den dort entspringenden Systemarterien abgeht.

Der sogenannte right-ventricle-to-pulmonary-artery-Shunt (RVPA-Shunt) wird entweder zwischen der rechten Kammer und der zentralen Pulmonalarterie (Sano-Shunt) oder der rechten Pulmonalarterie angelegt (Ziemer, Haverich 2010).

Im Gegensatz dazu unterteilt sich der systemico-pulmonale Shunt (SP-Shunt) - der eine Verbindung zwischen den Systemarterien und dem pulmonalen Gefäßbett schafft - noch weiter in den zentralen aortopulmonalen Shunt (zAP-Shunt) und den modifizierten Blalock-Taussig-Shunt (mBT-Shunt). Die Rohrprothese wird beim mBT-Shunt zwischen dem Truncus brachiocephalicus und der rechten Pulmonalarterie, beim zAP-Shunt meist zwischen Aorta ascendens und zentraler Pulmonalarterie angelegt (Ziemer, Haverich 2010).

Verschiedene Studien haben die Vor- und Nachteile der jeweiligen Shuntarten untersucht, wobei vor allem auf den standardmäßig verwendeten RVPA-Shunt und mBT-Shunt fokussiert wurde. Bei Vorliegen eines RVPA-Shunts bleibt der Systemdruck in der Diastole höher als beim mBT-Shunt, da bei letzterem das Blut in der Diastole weiter in das pulmonale Gefäßbett abfließt. Aus diesem Grund ist die diastolische Koronarperfusion beim RVPA-Shunt besser gewährleistet. Dem gegenüber steht allerdings die Notwendigkeit einer Ventrikulotomie bei der Anlage des RVPA-Shunts, die neben dem Risiko für eine ineffizientere Pumpfunktion der Kammer auch eine erhöhte Wahrscheinlichkeit für das Auftreten von Herzrhythmusstörungen mit sich bringt (Ohye et al. 2007).

Eine weitere Hypothese ist, dass der RVPA-Shunt durch einen vermehrten pulsatilen Blutfluss vorteilhafter für das Wachstum der Pulmonalarterien ist (Rumball et al. 2005), wobei diese Theorie in der Multicenter-Studie des Single Ventricle Reconstruction Trial nicht bewiesen werden konnte (Aiyagari et al. 2013) und diese Frage somit noch in Diskussion steht.

Uneinigkeiten bestehen ebenfalls bezüglich der Abhängigkeit der Überlebenswahrscheinlichkeit der Patienten von der Art des Shunts. In der Multicenter-Studie des Single Ventricle Reconstruction Trials wurde die vorher bereits schon mehrfach postulierte erhöhte postoperative Mortalität bei Vorliegen eines mBT-Shunt im Vergleich zum RVPA-Shunt erneut beschrieben. Die häufigste Todesursache war unabhängig von der Art des Shunts ein kardiovaskuläres Versagen. Ungeklärte Todesursachen stellten den zweithäufigsten Grund für das Versterben dar, wobei auch hier keine signifikanten Unterschiede zwischen den beiden Shuntarten festgestellt werden konnte (Ohye et al. 2012).

Ein großes Problem stellt die Interstage-Mortalität zwischen der Shunt-Operation und der PCPA dar. Als Ursache wird unter anderem die shuntabhängige Lungenperfusion mit der Gefahr des akuten Shuntverschlusses und die vermehrte Volumenbelastung des univentrikulären Herzens, sowie die ungünstigen hämodynamischen Bedingungen beim mBT-Shunt mit reduzierter Koronarperfusion in der Diastole angesehen (Ghanayem et al. 2012).

\subsubsection{Voraussetzungen für die Durchführung der partiellen cavopulmonalen Anastomose}

Um eine PCPA erfolgreich durchführen und ein optimales postoperatives Ergebnis erzielen zu können, sollten präoperativ einige hämodynamische Parameter des Patienten mittels Echokardiografie und Herzkatheteruntersuchung erhoben werden. 
Hierzu gehört zum einen die Evaluation der ventrikulären Funktion, der Mündung der Lungenvenen, der atrioventrikularen Klappenfunktion und des Vorhandenseins einer unrestriktiven Vorhoflücke.

Des Weiteren ist die Messung des mittleren pulmonalarteriellen Druckes und der Vorhofdrücke sowie die Beurteilung der Größe der Pulmonalarterien und die Aufdeckung möglicher

Pulmonalarterienstenosen von Bedeutung, um ggf. bereits vor der Durchführung der PCPA intervenieren zu können oder weitere Eingriffe wie eine Patcherweiterung der Pulmonalarterien im Rahmen der Operation planen zu können (Brown et al. 2003; Nakanishi 2005).

\subsection{Der Zeitpunkt der partiellen cavopulmonalen Anastomose}

\subsubsection{Bedeutung einer frühzeitigen Durchführung der partiellen cavopulmonalen Anastomose im klinischen Kontext}

Aufgrund der erhöhten Interstage-Mortalität zwischen der Shunt-Operation und der PCPA ist die Verbesserung der Hämodynamik durch die Anlage einer partiellen Trennung zwischen dem pulmonalen und systemischen Kreislauf nach Entfernung des lungenperfundierenden Shunts so früh wie möglich wünschenswert. Dies sorgt für eine verbesserte Koronarperfusion, eine Volumenentlastung des Systemventrikels und einen Schutz des pulmonalarteriellen Gefäßbettes vor flussbedingten erhöhten Druckwerten. Um von diesen Vorteilen so früh wie möglich profitieren zu können, neigt man dazu, die Operation im jüngeren Alter durchzuführen, wobei der optimale Zeitpunkt in der Literatur schon länger diskutiert wird (Cleuziou et al. 2008).

International wird die PCPA mittlerweile im Alter von drei bis sieben Monaten durchgeführt (Aiyagari et al. 2013).

Als eine große Limitation für die frühe Anlage der Anastomose in den ersten Wochen postnatal wird der erhöhte pulmonalvaskuläre Widerstand in diesem Alter angesehen (Jaquiss et al. 2004).

Es gibt einige Fallberichte und kleinere Studien, die über eine deutlich erhöhte Komplikationsrate und Mortalität nach Anlage einer frühzeitigen partiellen cavopulmonalen Anastomose berichten (Douglas et al. 1999; Lee et al. 2012; Reddy et al. 1997).

So wurde aufgrund einer erhöhten Inzidenz von Pulmonalarterienthrombosen und niedrigeren postoperativen mittleren arteriellen Sauerstoffsättigungen bei jüngeren Patienten zunächst von einer Anlage der Anastomose im Alter von unter vier Monaten abgeraten (Bradley et al. 1996).

In einer späteren Studie wurden in dieser Altersgruppe zwar noch längere und komplikationsreichere postoperative Intensiv- und Krankenhausaufenthalte (niedrigere arterielle Sauerstoffsättigung, längere Beatmungsdauer, längere Verweildauer einer Pleuradrainage) im Vergleich zu den älteren Patienten festgestellt, allerdings zeigte sich keine erhöhte Mortalität mehr (Jaquiss et al. 2004).

Zwei Jahre später veröffentlichte die gleiche Studiengruppe ergänzend, dass zum Zeitpunkt der Fontan-Komplettierung keine klinischen oder hämodynamischen Unterschiede zwischen den damals untersuchten Patienten bestanden und somit bis zu diesem Zeitpunkt keine Nachteile einer frühzeitigen Anlage der PCPA zu beobachten waren (Jaquiss et al. 2006).

Allerdings wurde eine erhöhte Mortalität bei Patienten, die bei Anlage der Glenn-Anastomose unter vier Monate alt waren, beobachtet (Nicolas et al. 2005). 
Im Vergleich zu Patienten, die zum Zeitpunkt der PCPA entweder unter oder über drei Monate alt waren, zeigte sich bei den jüngeren Patienten zwar eine längere Beatmungsdauer sowie ein längerer Aufenthalt auf der Intensivstation und der Klinik insgesamt, jedoch keine erhöhte Mortalität (Petrucci et al. 2010).

Auch in aktuellen Untersuchungen, die Daten der letzten 50 Jahre auswerteten, konnte kein Zusammenhang zwischen dem Alter zum Zeitpunkt des klassischen Glenn-Shunts und dem Überleben festgestellt werden (Zahr et al. 2016).

\subsubsection{Der Einfluss der partiellen cavopulmonalen Anastomose auf das Wachstum der Pulmonalarterien}

Im Alter von zwei bis vier Jahren wird die Fontan-Zirkulation durch die Anlage der TCPA komplettiert, wobei diese Operation im Deutschen Herzzentrum München standardmäßig im Alter von 18 Monaten bzw. bei einem Körpergewicht von zehn Kilogramm durchgeführt wird. Um einen erfolgreichen postoperativen Verlauf abschätzen zu können, wurde immer wieder versucht präoperative Risikofaktoren für ein Versagen der kompletten Kreislauftrennung zu definieren. Als ein negativer Einflussfaktor für eine erfolgreiche Fontan-Komplettierung wurden zum Beispiel zu kleine Pulmonalarterien angesehen (Knott-Craig et al. 1993). Ein fehlendes oder vermindertes Wachstum der Pulmonalarterien wird unter anderem durch den Wegfall des pulsatilen Blutflusses bzw. durch die niedrige Flussrate in die pulmonalen Gefäße nach Anlage der PCPA erklärt (Reddy et al. 1996; Reddy et al. 1995).

Das Verhalten des Pulmonalarterienwachstums nach Durchführung der PCPA wurde in mehreren Studien untersucht.

Um einen standardisierten Parameter für die Beurteilung der Größe der Pulmonalarterien bzw. deren Wachstum zu erlangen, wurden in der Literatur verschiedene Indices herangezogen, welche die Größe der Pulmonalarterien bezogen auf die Körperoberfläche des Patienten betrachten (Nakata et al. 1984; Reddy et al. 1996).

Zwar werden bei diesen Indices die Pulmonalarterien fälschlicherweise als exakte Kreise angesehen, jedoch kann man davon ausgehen, dass diese Betrachtungsweise realistischere Werte liefert, als die Verwertung des reinen zweidimensional gemessenen Durchmessers. Des Weiteren bieten diese Indices durch den Bezug auf die Körperoberfläche den Vorteil, eine Aussage über das gewichts- und größenadaptierte statt das reine Wachstum der Pulmonalarterien treffen zu können. Somit können Unterschiede in den somatischen Gegebenheiten und Entwicklungen zwischen den Patienten herausgefiltert werden.

Um eine bessere Vergleichbarkeit zwischen verschiedenen Studien zu erreichen, wurden die damals von Nakata et al. gewählten Messpunkte der Pulmonalarteriendurchmesser, kurz vor dem Abgang der jeweiligen Oberlappenarterie, in nachfolgenden Studien weitgehend beibehalten (Pruetz et al. 2009).

\subsubsection{Möglicher Einfluss des Alters zum Zeitpunkt der partiellen cavopulmonalen Anastomose auf das Wachstum der Pulmonalarterien - Ziel der Arbeit}

In mehreren Studien konnte ein fehlendes oder geringes Wachstum der Pulmonalarterien zwischen der PCPA und der TCPA gezeigt werden. 
Bereits 1993 wurde dieser Zusammenhang untersucht. In dieser kleinen Studie konnten keine signifikanten Änderungen des Nakata-Index festgestellt werden, als die vor der Durchführung der PCPA mit denen im Median 9,3 Monate postoperativ ermittelten Werten verglichen wurden (Chang et al. 1993). Drei Jahre später konnte eine Abnahme der Nakata-Indices und der Indices der linken Pulmonalarterie nach Anlage der bidirektionalen Glenn-Anastomose im Vergleich zu den Messungen vor der Operation gezeigt werden, auch wenn sich diese Ergebnisse als nicht signifikant herausstellten (Reddy et al. 1996).

Um den direkten Einfluss der PCPA und damit der Entfernung des pulsatilen Shuntflusses auf das Wachstum der Pulmonalarterien besser beurteilen zu können, führten Kansy et al. die Vermessungen der Pulmonalarterien sowohl kurz vor der PCPA als auch kurz vor der TCPA durch. Auch hier konnte der signifikante Rückgang der Nakata-Indices und damit das fehlende Wachstum der Pulmonalarterien gezeigt werden (Kansy et al. 2013).

Da durch ein Vorziehen der Anastomosenanlage der Shunt früher entfernt wird und damit auch die Dauer eines pulsatilen Blutflusses durch die Pulmonalarterien verkürzt wird, würde eine frühzeitige Durchührung der PCPA nach der Hypothese des wachstumsstimulierenden Effekts des pulsatilen Shuntflusses zu einer weiteren Einschränkung des Pulmonalarterienwachstums führen.

Um mehr über den Einfluss des Alters zum Zeitpunkt der bidirektionalen Glenn-Anastomose herausfinden zu können, verglichen Cleuziou et al. die kurz vor der PCPA und die kurz vor der TCPA gemessen Pulmonalarteriendurchmesser zwischen Patienten, die zum Zeitpunkt der PCPA älter bzw. jünger als sechs Monaten alt waren. Als Maß für die Größe der Pulmonalarterien wurde neben den reinen Durchmessern die z-scores berechnet, welche die gemessenen Werte mit denen von gesunden Kindern, die aber die gleiche Körperoberfläche aufweisen, in Verhältnis setzen. In beiden Studiengruppen zeigte sich zwischen den beiden Operationen eine Zunahme der Pulmonalarteriendurchmesser, aber ein Rückgang der Z-scores, was auf ein beeinträchtigtes Wachstum der Pulmonalarterien schließen ließ. Dabei war die linke Pulmonalarterie in beiden Gruppen stets kleiner als die rechte Pulmonalarterie. Die Durchmesser der Pulmonalarterien waren in der jüngeren Gruppe zu beiden Messpunkten signifikant kleiner als in der älteren Gruppe. Ein signifikanter Unterschied in den z-scores zwischen den beiden Altersgruppen bestand jedoch nur bezogen auf die Werte der linken Pulmonalarterien, gemessen vor der TCPA (Cleuziou et al. 2008).

Jaquiss et al. publizierten, dass Patienten, die bei der Durchführung der PCPA unter vier Monate alt waren, keine kleineren Pulmonalarteriendurchmesser vor der Fontan-Komplettierung aufwiesen, als die Patienten, die über vier Monate alt waren (Jaquiss et al. 2006).

1996 veröffentlichten Slavik et al. einen Bericht über vier Patienten, die vor dem vollendeten zweiten Lebensmonat eine PCPA erhielten. Dort konnte durch den Vergleich der Pulmonalarteriendurchmesser prä- und postoperativ ein Wachstum der Pulmonalarterien, aber auch ein komplizierterer postoperativer Verlauf beobachtet werden, auch wenn diese Ergebnisse durch die geringe Patientenzahl nur einen geringen repräsentativen Wert haben (Slavik et al. 1996). Dennoch ist die Kernfrage dieser Studie - „how young is too young?" (Slavik et al. 1996, S.78) - auch noch zwanzig Jahre später aktuell.

Zusammenfassend stellt die Durchführung der PCPA also einen wichtigen Zwischenschritt der Palliation eines univentrikulären Herzens auf dem Weg zur Fontan-Zirkulation dar. Durch eine frühzeitige Durchführung dieser Operation können die Patienten von der verbesserten Hämodynamik, wie der Volumenentlastung für den dominanten Ventrikel und - im Falle eines mBT-Shunts - der

\footnotetext{
${ }^{1}$ „Wie jung ist zu jung?“ (Übersetzung des Verfassers)
} 
verbesserten diastolischen Koronarperfusion profitieren. Außerdem wird somit die Dauer der risikoreichen Interstage-Periode zwischen der Shunt-Operation und der Anastomosenanlage reduziert. Der erhöhte pulmonalvaskuläre Widerstand postnatal stellt sich jedoch als limitierender Faktor für die frühzeitige Durchführung der PCPA dar, ebenso wie die Möglichkeit des gehemmten Wachstum der Pulmonalarterien durch den frühen Wegfall des pulsatilen Shuntflusses.

Das Ziel dieser Arbeit war es, den Einfluss einer frühzeitigen PCPA im Alter von unter drei Monaten auf das Wachstum der Pulmonalarterien zu untersuchen und somit einen Beitrag zu den Überlegungen einer noch früheren Durchführung dieser Operation zu leisten.

\section{Material und Methoden}

\subsection{Patientenkollektiv: Rekrutierung und Gruppenbildung}

Das Patientenkollektiv $(\mathrm{n}=240)$ wurde retrospektiv zusammengestellt und besteht aus Patienten, bei denen zwischen Anfang 2006 und Mitte 2014 eine PCPA am Deutschen Herzzentrum München durchgeführt wurde. 17 Patienten waren zu dem Zeitpunkt der Operation älter als ein Jahr, drei Patienten erhielten eine 1 1/2-Korrektur, bei der zusätzlich zu dem Anschluss der oberen Hohlvene an die rechte Pulmonalarterie auch ein Blutfluss vom rechten Ventrikel über die Pulmonalklappe in das Lungengefäßbett stattfindet, und drei weitere Patienten wiesen eine Azygoskontinuität auf, bei der mit Ausnahme des Blutes aus den Lebervenen das gesamte venöse Blutvolumen über die obere Hohlvene in den rechten Vorhof mündet. Da die Eigenschaften des Blutflusses dieser 23 Patienten zu höheren Sauerstoffsättigungen führen und dies zu Verfälschungen der Ergebnisse führen könnte, wurden sie aus der folgenden Analyse ausgeschlossen.

Die restlichen 217 Patienten wurden anhand ihres Alters zum Zeitpunkt der Operation in drei Gruppen eingeteilt, um das Wachstum der Pulmonalarterien sowie verschiedene hämodynamische und postoperative Daten zwischen den drei Altersgruppen vergleichen zu können. Durch das Studium von Patientenakten und Angiografien konnten die verschiedenen Patientendaten erhoben werden. Wegen fehlender Daten oder nicht auswertbarer Angiografien bei retrospektiver Datenerhebung, variiert die Patientenzahl pro Altersgruppe bei den verschiedenen Parametern. Die genaue Anzahl der verwendeten Daten können der entsprechenden Grafik oder Tabelle entnommen werden. Im Folgenden werden nun die Methoden der Datenerhebung erläutert.

\subsection{Datenerhebung}

\subsubsection{Erhebung der deskriptiven Daten}

Das Geburtsdatum, die Zeitpunkte der PCPA und der TCPA sowie der jeweiligen präoperativen Herzkatheteruntersuchung wurden retrospektiv aus den Patientenakten erhoben. Aus diesen stammen ebenfalls die Diagnosen der zu Grunde liegenden Herzfehler und die Eigenschaften des angelegten Shunts.

Die Größe und das Gewicht der Patienten wurde bei Aufnahme in die Klinik zur Operation bzw. bei dauerhaft stationären Patienten zum Zeitpunkt der PCPA bzw. der TCPA gemessen. Diese Angaben wurden ebenfalls den Patientenakten entnommen. 


\subsubsection{Erhebung der hämodynamischen Daten}

Die hämodynamischen Daten sowie die Körperoberfläche der Patienten wurden retrospektiv aus den Herzkatheterprotokollen übernommen.

Folgende hämodynamische Daten wurden vor der PCPA und vor der TCPA für die Analyse erhoben: der mittlere pulmonalarterielle Druck (PAP) in Millimeter-Quecksilbersäule (mmHg), der enddiastolische Ventrikeldruck (VEDP) in $\mathrm{mmHg}$, der rechtsatriale (RAP) und linksatriale (LAP) Druck in mmHg, wobei bei unrestriktiver Vorhoflücke nach Atrioseptektomie der rechtsatriale dem linksatrialen Druck entspricht. Der transpulmonale Gradient (TPG) wurde aus der Differenz des mittleren pulmonalarteriellen und des mittleren linksatrialen Druck berechnet.

Die Druckregistrierung geschah über den flüssigkeitsgefüllten Katheter mit einem amplituden- und frequenzgesteuerten Aufnahmesystem am Ende der Flüssigkeitssäule und wird in mmHg angegeben.

\subsubsection{Vermessung der Pulmonalarterien und Berechnung der Indices}

Die Angiografien wurden mit dem Programm Artis zee/zeego Softwareversion VC14 der Firma Siemens registriert. Für die Darstellung der Pulmonalarterien erfolgte eine Angiografie mittels eines Benson-Hennefee oder Amplatzer rechtskoronaren Katheters vor der PCPA und über eine $4 \mathrm{~F}$ Schleuse vor der TCPA mit dem Kontrastmittel Imeron® 350 (Bracco, Deutschland).

Durch das digitale Studium der in den Herzkatheteruntersuchungen gewonnenen Angiografien mit Hilfe des Programms DIMARS ${ }^{\circledR}$ für Microsoft Windows ${ }^{\circledR}$ wurden retrospektiv die Durchmesser der Pulmonalarterien vor dem Abgang der jeweiligen Oberlappenarterie vor PCPA und vor TCPA gemessen (siehe Abbildung 1). Die Messung erfolgte in einer anterior-posterioren Projektion während der Systole. 


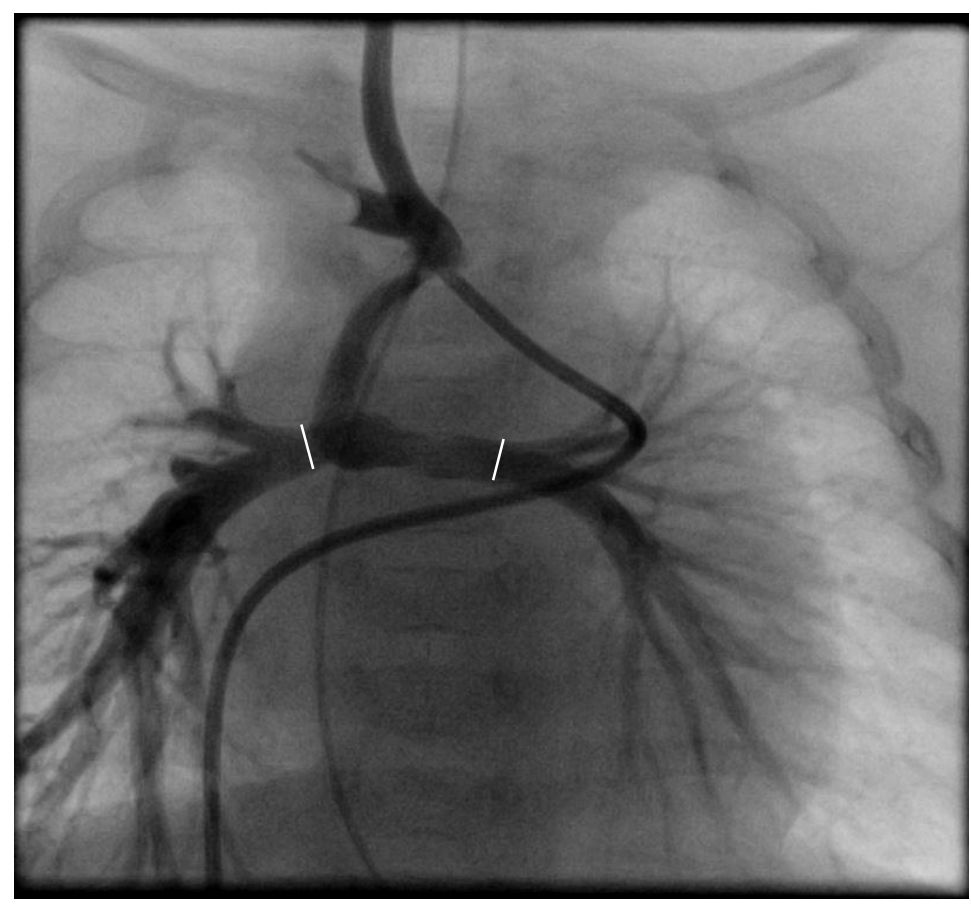

Abbildung 1: Schematische Darstellung der Messung der Pulmonalarterien

Exemplarisches Standbild einer angiografischen Darstellung der Pulmonalarterien vor Durchführung der partiellen cavopulmonalen Anastomose im Computerprogramm DIMARS ${ }$ für Microsoft Windows ${ }^{\circledR}$. Die weißen Linien kennzeichnen schematisch den Ort und die Art der Messung des Durchmessers der Pulmonalarterien. Es wurde auf eine senkrechte Linienführung kurz vor dem Abgang der jeweiligen Oberlappenarterie geachtet.

Um eine gute Vergleichbarkeit mit der Literatur zu gewährleisten, wurde der Nakata-Index berechnet, der sich aus der Summe der Querschnittsflächen beider Pulmonalarterien geteilt durch die Körperoberfläche (KOF) des Patienten zusammensetzt. Die Querschnitte wurden hierbei als Kreise betrachtet, um die entsprechende Flächenformel verwenden zu können (Nakata et al. 1984).

$$
A(P A)=\pi \times\left(\frac{d(P A)}{2}\right)^{2}
$$

Formel 1: Berechnung der Fläche einer Pulmonalarterie

Berechnung der Fläche (A) einer Pulmonalarterie (PA) in Quadratmillimetern anhand des gemessenen Durchmessers (d) in Millimetern.

$$
\text { Nakata }- \text { Index }=\frac{A(L P A)+A(R P A)}{K O F}
$$

Formel 2: Berechnung des Nakata-Index

Berechnung des Nakata-Index in Quadratmillimetern $\left(\mathrm{mm}^{2}\right)$ pro Quadratmeter $\left(\mathrm{m}^{2}\right)$ anhand der Fläche (A) der linken (LPA) und rechten (RPA) Pulmonalarterie in $\mathrm{mm}^{2}$ und der Körperoberfläche (KOF) des Patienten in $\mathrm{m}^{2}$ zum Zeitpunkt der Durchführung der Angiografie.

Des Weiteren wurden neben den reinen Durchmessern auch die einzelnen Flächen-Indices der jeweiligen Pulmonalarterie berechnet, die sich aus der errechneten Fläche einer Pulmonalarterie geteilt durch die Körperoberfläche des Patienten zusammensetzt (Reddy et al. 1996). 


$$
\text { Flächen }- \text { Index } P A=\frac{A(P A)}{K O F}
$$

Formel 3: Berechnung des Flächen-Index einer Pulmonalarterie

Berechnung des Flächen-Index in Quadratmillimetern $\left(\mathrm{mm}^{2}\right)$ pro Quadratmeter $\left(\mathrm{m}^{2}\right)$ einer Pulmonalarterie (PA) anhand der Fläche (A) der jeweiligen Pulmonalarterie in $\mathrm{mm}^{2}$ und der Körperoberfläche (KOF) des Patienten in $\mathrm{m}^{2}$ zum Zeitpunkt der Durchführung der Angiografie.

Außerdem wurde dem Herzkatheterprotokoll entnommen, ob eine Stenose einer oder beider Pulmonalarterien und/oder eine Stenose des lungenperfundierenden Shunts vorlagen.

\subsubsection{Erhebung der perioperativen und postoperativen Daten}

Folgende peri- und postoperative Daten wurden retrospektiv durch das Studium der Patientenakten ermittelt: Dauer der Zeit an der Herz-Lungen-Maschine in Minuten (min), totale postoperative Beatmungsdauer in Stunden (h), über $24 \mathrm{~h}$ gemittelte arterielle Sauerstoffsättigung $\left(\mathrm{SaO}_{2}\right)$ in den ersten $24 \mathrm{~h}, 48 \mathrm{~h}$ und $72 \mathrm{~h}$ postoperativ in Prozent (\%), über $24 \mathrm{~h}$ gemittelter pulmonalarterieller Druck (PAP) in den ersten $24 \mathrm{~h}, 48 \mathrm{~h}$ und $72 \mathrm{~h}$ postoperativ in $\mathrm{mmHg}$, zentraler Venendruck (ZVD) in $\mathrm{mmHg}$ und transpulmonaler Gradient (TPG) in mmHg $24 \mathrm{~h}$ und $48 \mathrm{~h}$ postoperativ, Dauer des Sauerstoffbedarfs $\left(\mathrm{O}_{2}\right.$-Bedarf), des Aufenthaltes auf der Intensivstation in Tagen (d) und des Aufenthalts im Krankenhaus in Tagen, transkutane Sauerstoffsättigung $\left(\mathrm{tcSO}_{2}\right)$ bei Entlassung in Prozent und Datum der letzten ambulanten Vorstellung oder ggf. Todeszeitpunkt.

Der pulmonale Mitteldruck wurde über einen zentralen Venenkatheter (ZVK) in der V. jugularis, der zentrale Venendruck über einen ZVK in der V. femoralis mittels Druckaufnehmer gemessen und der transpulmonale Gradient daraus berechnet. Bei einer unrestriktiven Vorhoflücke (präoperativ im Herzkatheter untersucht) entspricht der Druck im rechten Vorhof dem im linken Vorhof, sodass mit dem zentralen Venendruck der Druck im linken Vorhof abgeschätzt werden konnte.

\subsection{Statistische Auswertung}

Die Daten wurden mittels IBM SPSS Statistics 22® für Microsoft Windows® ausgewertet.

Bei normalverteilten, intervallskalierten Variablen wurden die Mittelwerte durch den t-Test auf signifikante Unterschiede untersucht. Lag keine Normalverteilung vor, wurden diese mittels des nicht parametrischen Mann-Whitney-U-Testes ermittelt.

Die Mittelwerte der nominalskalierten Variablen wurden mit dem $\mathrm{x}^{2}$-Test oder dem Fischer-ExaktTest verglichen.

Mögliche Einflussfaktoren auf die Indices der Pulmonalarterien wurden mit Hilfe der linearen Regressionsanalyse untersucht.

Die Überlebensstatistik wurde mittels Kaplan-Meier-Kurven dargestellt und signifikante Unterschiede durch den Log-Rank-Test ermittelt.

Durch die Cox-Regression wurde der mögliche Einfluss von Variablen auf die Mortalität untersucht. 
Das Signifikanzniveau lag bei einem $\mathrm{p}$-Wert von $<0,05$, sowie einem Konfidenzintervall von $95 \%$.

Sämtliche Grafiken wurden mit dem Programm IBM SPSS Statistics 22® für Microsoft Windows® erstellt.

\section{Ergebnisse}

\subsection{Patientenkollektiv: Deskriptive Statistik}

Die Patienten wurden anhand ihres Alters zum Zeitpunkt der PCPA in drei Gruppen eingeteilt. Als „frühzeitig“ operiert galten Patienten, die bei dieser Operation unter drei Monate (90 Tage oder jünger) alt waren. Das mittlere Alter zum Zeitpunkt der PCPA lag bei 75 Tagen (2,5 Monate), wobei der jüngste Patient 50 Tage (1,7 Monate) alt war. Als „elektiv“ wurden Patienten bezeichnet, die zum Zeitpunkt der Durchführung der PCPA zwischen drei und sechs Monate alt waren (91-182 Tage; mittleres Alter 125 Tage = 4,2 Monate). Die Bezeichnung „spät“ erhielt die Gruppe mit Patienten, deren Alter über sechs, aber unter zwölf Monate lag (>182 Tage; mittleres Alter 236 Tage = 7,9 Monate; Maximum 348 d = 11,6 Monate).

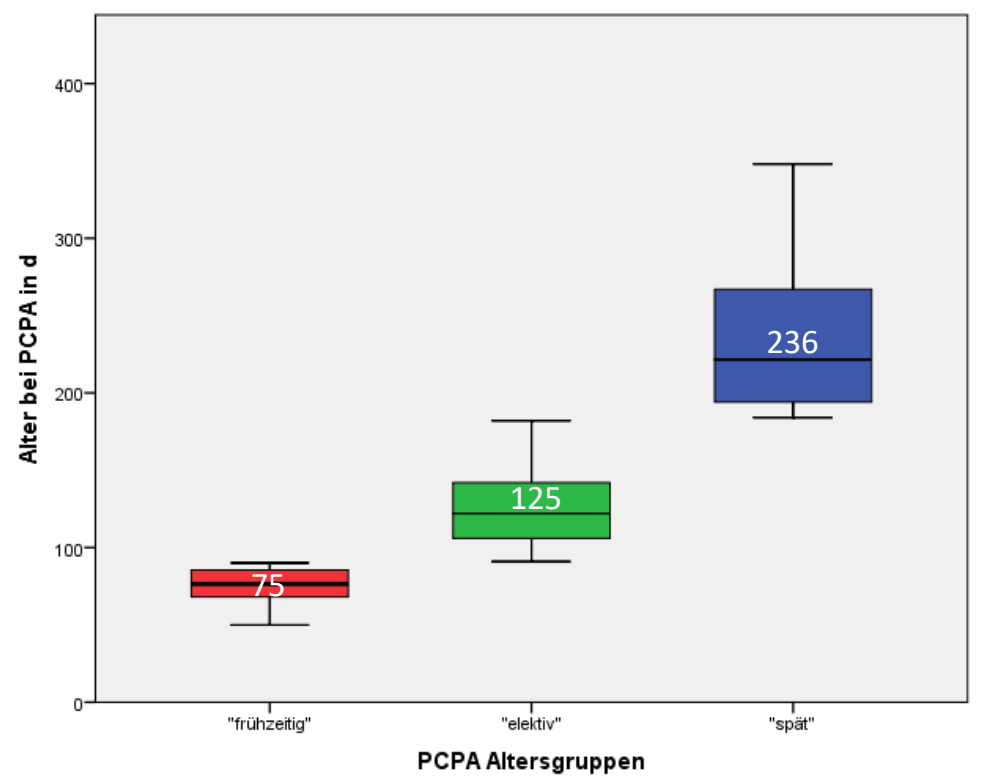

Abbildung 2: Alter der Patienten zum Zeitpunkt der PCPA

Alter der Patienten zum Zeitpunkt der partiellen cavopulmonalen Anastomose (PCPA) in den drei Altersgruppen: Als „frühzeitig“ wird ein Alter von kleiner gleich 90 Tagen (d) (rot), als „,elektiv“ zwischen 91 und 182 d (grün) und als „spät“ von über 182 d (blau) bezeichnet. Die weißen Zahlen in den Boxplots entsprechen dem mittleren Alter der jeweiligen Altersgruppe in d. Anzahl der Patienten in der „frühzeitigen“ Gruppe $n=40$, in der „elektiven“ Gruppe n = 137 und in der ,späten“ Gruppe n $=40$.

Nach der PCPA erhielten 13 Patienten bei therapieresistenter, lebensbedrohlicher postoperativer Hypoxie zusätzlich noch einen Shunt zum linken Lungengefäßbett als ,,way-out-Strategie“. Diese Patienten wurden bei den Berechnungen nach Durchführung der PCPA aus der Studie ausgeschlossen, da durch den zusätzlichen pulsatilen Fluss über den Shunt das Wachstum der Pulmonalarterien beeinflusst hätte werden können. Das mittlere Alter dieser Patientengruppe lag bei 117 Tagen $(=3,9$ Monate; Minimum 55 Tage, Maximum 214 Tage), wobei 23,1\% (drei Patienten) 90 Tage oder jünger, 
69,2\% (neun Patienten) zwischen drei und sechs Monaten und 7,2 \% (ein Patient) älter als sechs Monate waren.

Somit befanden sich bei den Berechnungen nach der PCPA in der ,frühzeitigen“ Gruppe $n=37$, in der „elektiven“ Gruppe n = 128 und in der „späten“ Gruppe n = 39 Patienten. Bei 140 Patienten wurde innerhalb des Studienzeitraums eine TCPA durchgeführt.

Die ,frühzeitige“ Durchführung der PCPA stellte keine elektive Operation dar, sondern war eine notwendige Intervention aufgrund des klinischen Zustandes der Patienten. Aufgrund von anhaltender Gedeihstörung und Trinkschwäche (mit der Notwendigkeit der Teil- bzw. Vollsondierung), anhaltendem Sauerstoffbedarf, anhaltender Zyanose mit einer transkutanen Sauerstoffsättigung von unter $75 \%$, eingeschränkter Ventrikelfunktion oder höhergradiger atrioventrikularer Klappeninsuffizienz mit der Notwendigkeit einer intravenösen medikamentösen Therapie, konnten die Patienten nicht in die ambulante Betreuung entlassen werden.

Der häufigste zugrunde liegende Herzfehler im Patientenkollektiv war das HLHS (41,5\%), gefolgt von der Trikuspidalatresie (13,8\%) und dem Double-inlet-left-ventricle (10,6\%). Andere Vitien $(34,1$ $\%$ ), welche die univentrikuläre Palliation erforderlich machten, waren unter anderem der doubleoutlet-right-ventricle, der imbalancierte komplette atrioventrikuläre Septumdefekt, die kritische Aortenklappenstenose, grenzwertig kleine Linksstrukturen, der Shone-Komplex, das HeterotaxieSyndrom und die Pulmonalatresie.

Die Verteilung der unterschiedlichen Shuntarten in den drei Altersgruppen kann Abbildung 2 entnommen werden.

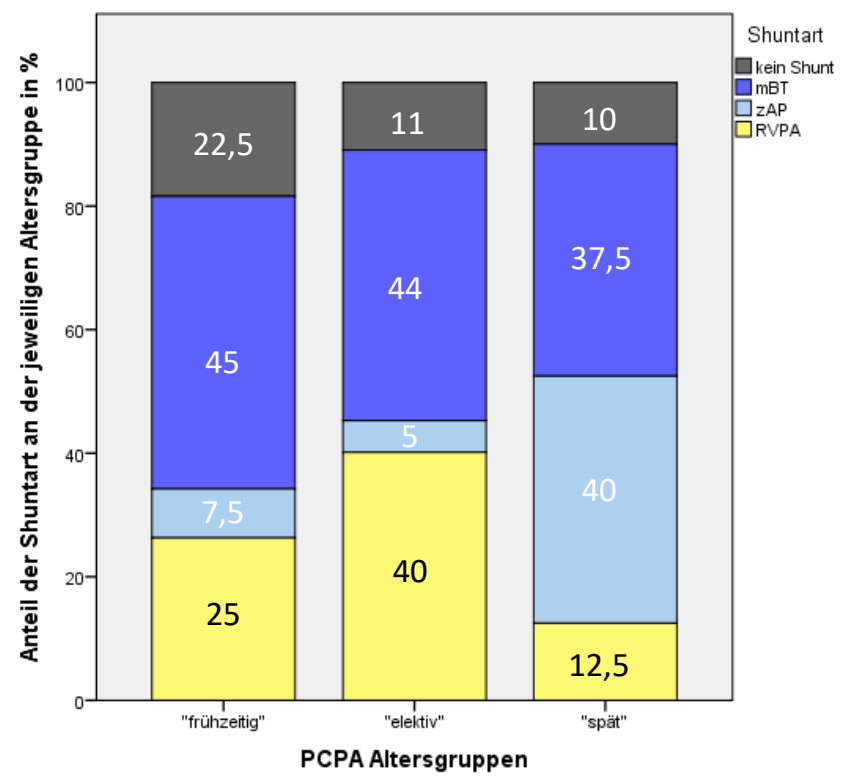

Abbildung 3: Verteilung der verschiedenen Shuntarten in den drei Altersgruppen

Balkendiagramm zur Repräsentation der Verteilung der Arten des lungenperfundierenden Shunts in den drei Altersgruppen. Als ,frühzeitig“ wird ein Alter bei der partiellen cavopulmonalen Anastomose von kleiner gleich 90 Tagen (d) bezeichnet, als „,elektiv“ ein Alter zwischen 91 und 182 d und als „spät“ ein Alter von über 182 d. Kein Shunt (grau), modifizierter Blalock-Taussig-Shunt (mBT: mittelblau), zentraler aortopulmonaler Shunt (zAP: hellblau), right-ventricle-to-pulmonary-artery-shunt (RVPA: gelb). Die weißen Zahlen in den Balken entsprechen dem Anteil der jeweiligen Shuntart an der Altersgruppe in Prozent (\%). Anzahl der Patienten pro Shuntart jeweils für die „frühzeitige“, die „elektive“ und die „späte“ Gruppe: kein Shunt n = 9, $\mathrm{n}=15$ und $\mathrm{n}=4$; mBT-Shunt $\mathrm{n}=$ $18, \mathrm{n}=60$ und $\mathrm{n}=15 ; \mathrm{zAP}-$ Shunt $\mathrm{n}=3, \mathrm{n}=7$ und $\mathrm{n}=16 ; \mathrm{RVPA}-$ Shunt $\mathrm{n}=10, \mathrm{n}=15$ und $\mathrm{n}=4$. 
Aufgrund der sehr ähnlichen hämodynamischen Eigenschaften des zAP- und des mBT-Shunts wurden diese beiden Shuntarten zu der Gruppe des SP-Shunts zusammengefasst. In der Gruppe der „Späten“ war der Anteil der Patienten mit einem SP-Shunt signifikant höher und der Anteil der Patienten mit einem RVPA-Shunt signifikant niedriger als in der Gruppe der „Elektiven“ (SP: $p=0,004 ;$ RVPA: $p=$ 0,001). Zwischen den anderen Altersgruppen fanden sich keine signifikanten Unterschiede.

Die deskriptive Statistik der jeweiligen Altersgruppe kann den Abbildungen 4 bis 6 (vor PCPA), sowie den Abbildungen 7 bis 10 (vor TCPA) entnommen werden. Die mittlere Körpergröße, Körpergewicht und Körperoberfläche unterschieden sich zum Zeitpunkt der Durchführung der PCPA zwischen jeder der drei Gruppen signifikant $(\mathrm{p}<0,001)$.

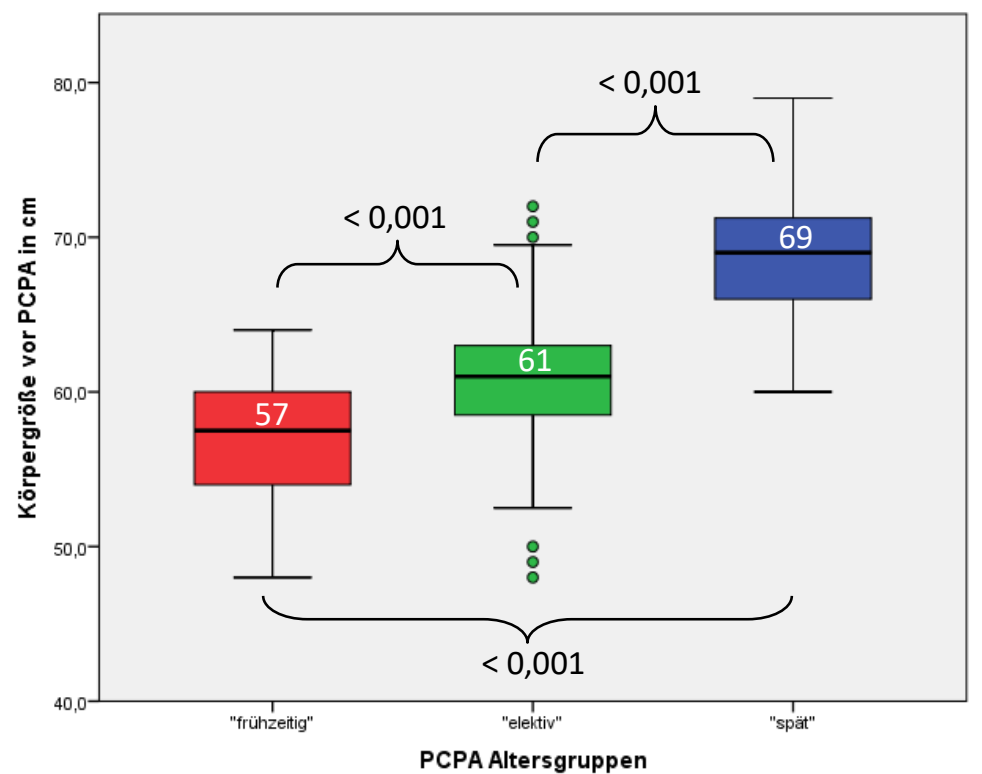

Abbildung 4: Vergleich der Körpergröße vor PCPA zwischen den drei Altersgruppen

Vergleich der Körpergröße vor der partiellen cavopulmonalen Anastomose (PCPA) zwischen den drei Altersgruppen: Als „frühzeitig“ wird ein Alter bei PCPA von kleiner gleich 90 Tagen (d) (rot), als ,,elektiv“ zwischen 91 und 182 d (grün) und als „spät“ von über 182 d (blau) bezeichnet. Die weißen Zahlen in den Boxplots entsprechen der mittleren Körpergröße der jeweiligen Altersgruppe in Centimetern (cm). Signifikante

Unterschiede zwischen zwei Gruppen sind durch eine Klammer gekennzeichnet, der entsprechende signifikante pWert steht darüber. Das Signifikanzniveau lag bei $\mathrm{p}<0,05$. Anzahl der Patienten in der "frühzeitigen“ Gruppe $\mathrm{n}=$ 40 , in der ,elektiven“ Gruppe $n=137$ und in der ,späten“ Gruppe n $=40$. 


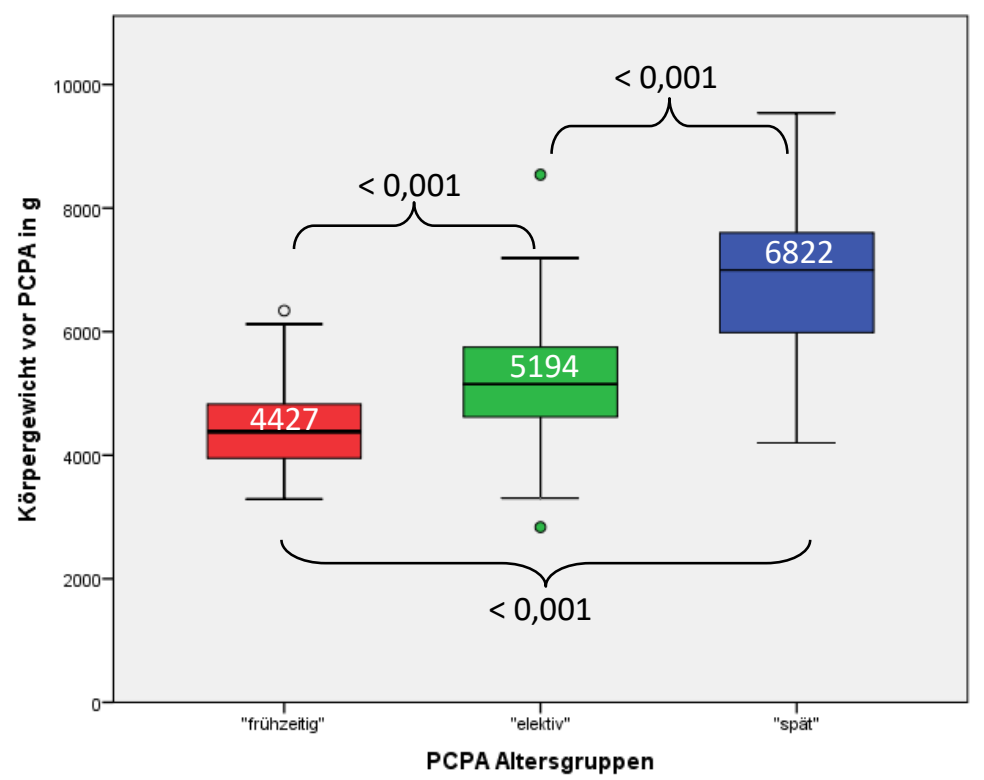

Abbildung 5: Vergleich des Körpergewichts vor PCPA zwischen den drei Altersgruppen

Vergleich des Körpergewichts vor der partiellen cavopulmonalen Anastomose (PCPA) zwischen den drei Altersgruppen: Als „frühzeitig“ wird ein Alter bei PCPA von kleiner gleich 90 Tagen (d) (rot), als „elektiv“ zwischen 91 und $182 \mathrm{~d}$ (grün) und als „spät“ von über $182 \mathrm{~d}$ (blau) bezeichnet. Die weißen Zahlen in den Boxplots entsprechen dem mittleren Körpergewicht der jeweiligen Altersgruppe in Gramm (g). Signifikante Unterschiede zwischen zwei Gruppen sind durch eine Klammer gekennzeichnet, der entsprechende signifikante p-Wert steht darüber. Das Signifikanzniveau lag bei p $<0,05$. Anzahl der Patienten in der „,frühzeitigen“ Gruppe $n=40$, in der „elektiven“ Gruppe $\mathrm{n}=137$ und in der „späten“ Gruppe $\mathrm{n}=40$.

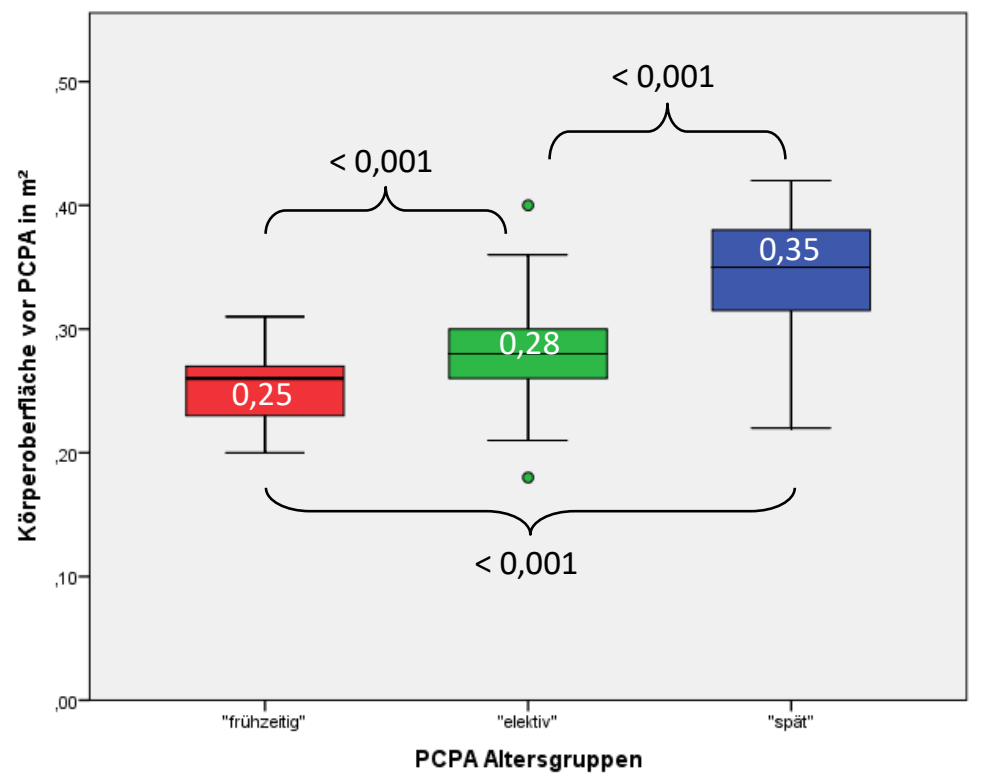

Abbildung 6: Vergleich der Körperoberfläche vor PCPA zwischen den drei Altersgruppen

Vergleich der Körperoberfläche vor der partiellen cavopulmonalen Anastomose (PCPA) zwischen den drei Altersgruppen: Als „frühzeitig“ wird ein Alter bei PCPA von kleiner gleich 90 Tagen (d) (rot), als „elektiv“ zwischen 91 und $182 \mathrm{~d}$ (grün) und als „spät“ von über $182 \mathrm{~d}$ (blau) bezeichnet. Die weißen Zahlen in den Boxplots entsprechen der mittleren Körperoberfläche der jeweiligen Altersgruppe in Quadratmetern $\left(\mathrm{m}^{2}\right)$. Signifikante Unterschiede zwischen zwei Gruppen sind durch eine Klammer gekennzeichnet, der entsprechende signifikante pWert steht darüber. Das Signifikanzniveau lag bei $\mathrm{p}<0,05$. Anzahl der Patienten in der „frühzeitigen“ Gruppe $\mathrm{n}=$ 40 , in der „elektiven“ Gruppe $\mathrm{n}=137$ und in der „späten“ Gruppe $\mathrm{n}=40$. 
Vor der TCPA stellte sich nur die mittlere Körperoberfläche der „Frühzeitigen“ als signifikant größer im Vergleich zu den „Elektiven“ dar ( $\mathrm{p}=0,042)$.

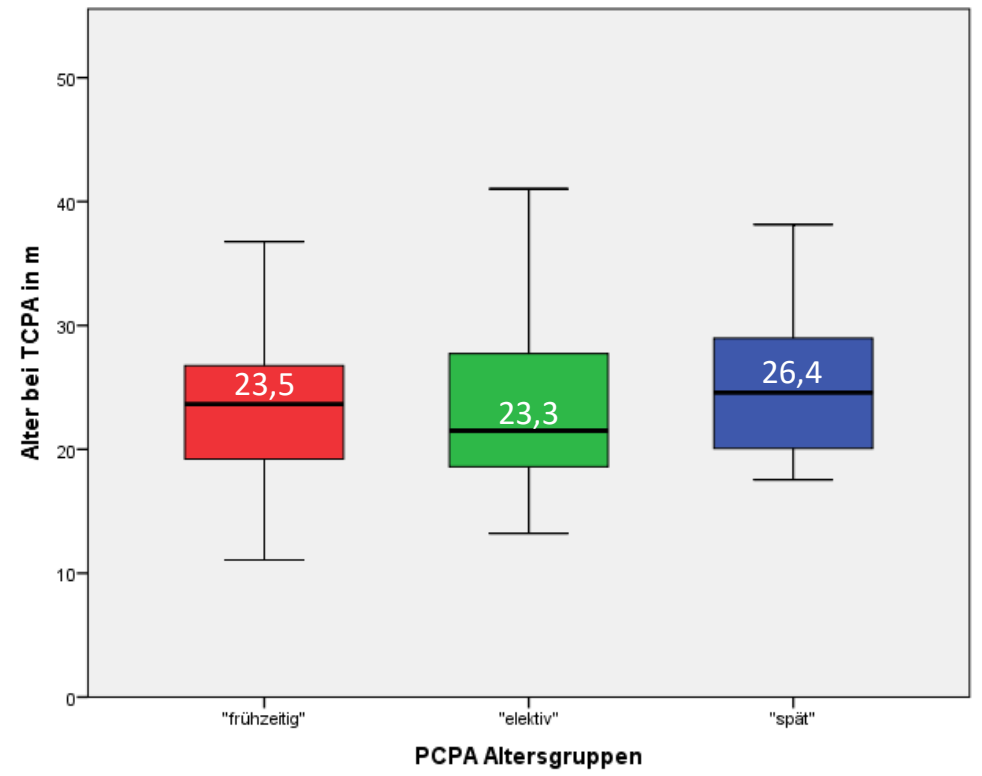

Abbildung 7: Alter der Patienten zum Zeitpunkt der TCPA

Alter der Patienten zum Zeitpunkt der totalen cavopulmonalen Anastomose (TCPA) in den drei Altersgruppen: Als „frühzeitig“ wird ein Alter bei der partiellen cavopulmonalen Anastomose (PCPA) von kleiner gleich 90 Tagen (d) (rot), als „elektiv“ zwischen 91 und 182 d (grün) und als „spät“ von über 182 d (blau) bezeichnet. Die weißen Zahlen in den Boxplots entsprechen dem mittleren Alter der jeweiligen Altersgruppe in Monaten (m). Es konnten keine signifikanten Unterschiede zwischen den Altersgruppen ermittelt werden. Das Signifikanzniveau lag bei p < 0,05. Anzahl der Patienten in der ,frühzeitigen“ Gruppe $n=24$, in der ,elektiven“ Gruppe $n=82$ und in der „späten“ Gruppe n = 31 .

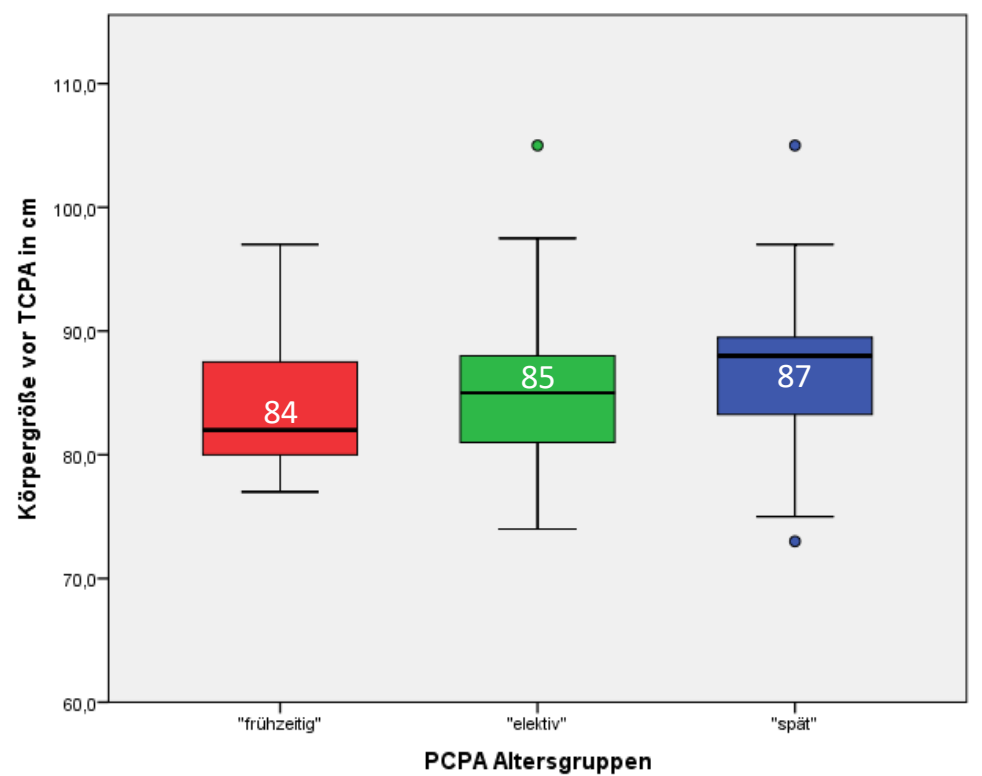

Abbildung 8: Vergleich der Körpergröße vor TCPA zwischen den drei Altersgruppen

Vergleich der Körpergröße vor der totalen cavopulmonalen Anastomose (TCPA) zwischen den drei Altersgruppen: Als „frühzeitig“ wird ein Alter bei der partiellen cavopulmonalen Anastomose (PCPA) von kleiner gleich 90 Tagen (d) (rot), als „elektiv“ zwischen 91 und 182 d (grün) und als „spät“ von über 182 d (blau) bezeichnet. Die weißen Zahlen in den Boxplots entsprechen der mittleren Körpergröße der jeweiligen Altersgruppe in Centimetern (cm). Es konnten keine signifikanten Unterschiede zwischen den Altersgruppen ermittelt werden. Das Signifikanzniveau lag bei $p<0,05$. Anzahl der Patienten in der „frühzeitigen“ Gruppe $n=24$, in der ,elektiven“ Gruppe $n=77$ und in der ,späten“ Gruppe n = 31 . 


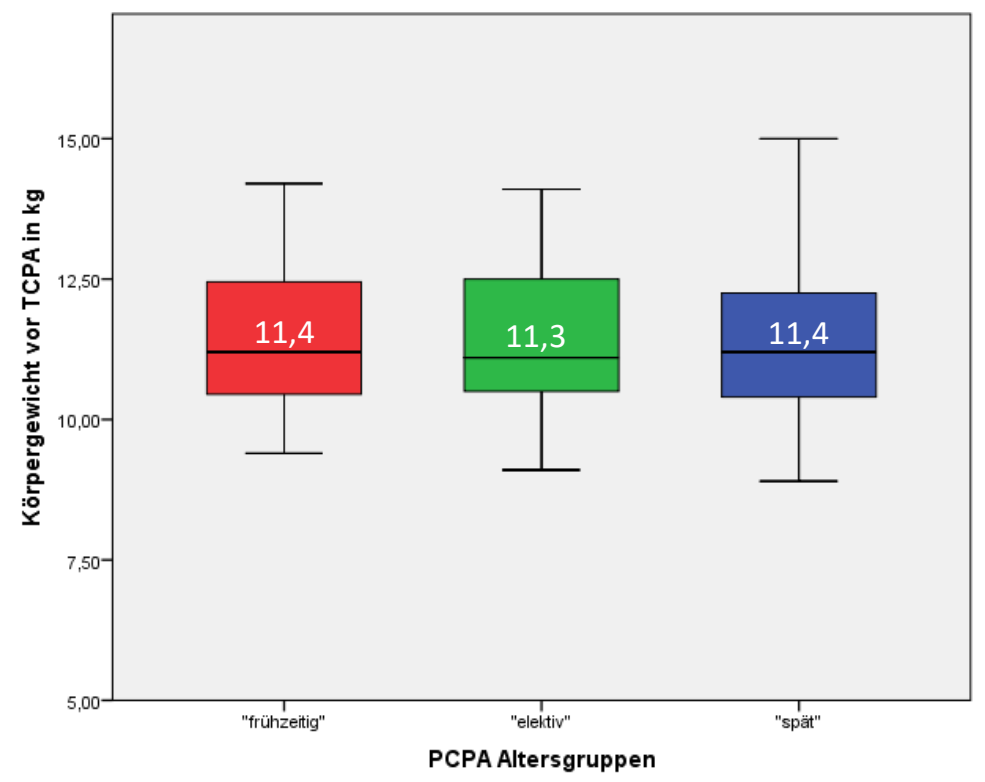

Abbildung 9: Vergleich des Körpergewichts vor TCPA zwischen den drei Altersgruppen

Vergleich des Körpergewichts vor der totalen cavopulmonalen Anastomose (TCPA) zwischen den drei Altersgruppen: Als „frühzeitig“ wird ein Alter bei der partiellen cavopulmonalen Anastomose (PCPA) von kleiner gleich 90 Tagen (d) (rot), als „elektiv“ zwischen 91 und $182 \mathrm{~d}$ (grün) und als „spät“ von über $182 \mathrm{~d}$ (blau) bezeichnet. Die weißen Zahlen in den Boxplots entsprechen dem mittleren Körpergewicht der jeweiligen Altersgruppe in Kilogramm (kg). Es konnten keine signifikanten Unterschiede zwischen den Altersgruppen ermittelt werden. Das Signifikanzniveau lag bei $\mathrm{p}<0,05$. Anzahl der Patienten in der "frühzeitigen“ Gruppe $\mathrm{n}=$ 24 , in der „elektiven“ Gruppe $\mathrm{n}=77$ und in der „späten“ Gruppe n $=31$.

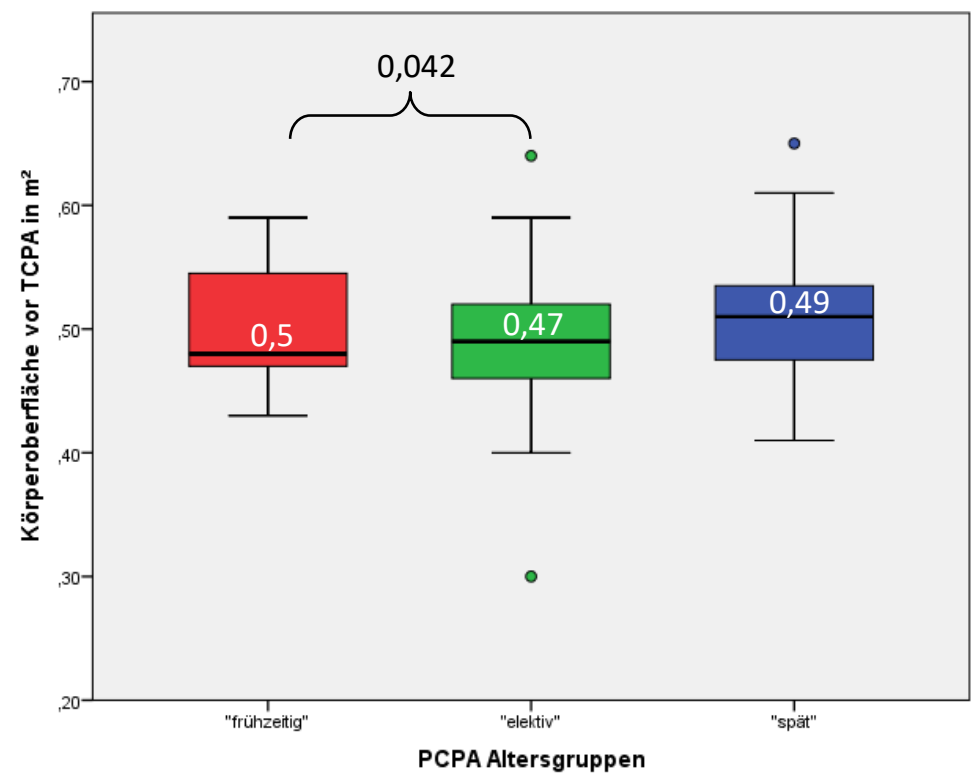

Abbildung 10: Vergleich der Körperoberfläche vor TCPA zwischen den drei Altersgruppen

Vergleich der Körperoberfläche vor der totalen cavopulmonalen Anastomose (TCPA) zwischen den drei Altersgruppen: Als „frühzeitig“ wird ein Alter bei der partiellen cavopulmonalen Anastomose (PCPA) von kleiner gleich 90 Tagen (d) (rot), als „elektiv“ zwischen 91 und 182 d (grün) und als „spät“ von über 182 d (blau) bezeichnet. Die weißen Zahlen in den Boxplots entsprechen der mittleren Körperoberfläche der jeweiligen Altersgruppe in $\mathrm{m}^{2}$. Signifikante Unterschiede zwischen zwei Gruppen sind durch eine Klammer gekennzeichnet, der entsprechende signifikante $\mathrm{p}$-Wert steht darüber. Das Signifikanzniveau lag bei p < 0,05. Anzahl der Patienten in der frühzeitigen Gruppe $\mathrm{n}=24$, in der ,elektiven“ Gruppe $\mathrm{n}=77$ und in der „,späten“ Gruppe $\mathrm{n}=31$. 
Die Zeit zwischen der Durchführung der PCPA und der TCPA lag im Mittel bei 18,6 Monaten (Standardabweichung 7,5 Monate). Hierbei konnte kein signifikanter Unterschied zwischen den drei Altersgruppen festgestellt werden.

\subsection{Herzkatheteruntersuchung}

Die standardmäßig durchgeführte Herzkatheteruntersuchung erfolgt im Median 5 Tage vor der Anlage der PCPA (Minimum 1 d, Maximum 173 d) bzw. im Median 5 Tage vor Anlage der TPCA (Minimum $1 \mathrm{~d}$, Maximum 254 d). Es gab keine signifikanten Unterschiede zwischen den drei Altersgruppen.

\subsubsection{Hämodynamische Werte}

Zwischen den drei Altersgruppen zeigten sich in den vor der PCPA erhobenen hämodynamischen Parametern keine signifikanten Unterschiede des mittleren enddiastolischen Druckes im Ventrikel (VEDP), des mittleren pulmonalarteriellen Druckes (PAP), des mittleren Druckes im linken (LAP) bzw. rechten Atriums (RAP) und des daraus resultierenden mittleren transpulmonalen Gradienten (TPG). Die Mittelwerte und Signifikanzwerte können Tabelle 3 entnommen werden.

Hämodynamische Parameter vor Durchführung der PCPA im Vergleich zwischen den drei Altersgruppen

\begin{tabular}{lccc}
\hline & „frühzeitig“ & „elektiv“ & „spät“ \\
\hline PAP & $15(5)$ & $15(5)$ & $16(5)$ \\
\hline LAP & $6(3)$ & $7(3)$ & $6(2)$ \\
\hline RAP & $6(3)$ & $6(3)$ & $6(2)$ \\
\hline TPG & $10(5)$ & $8(4)$ & $10(5)$ \\
\hline VEDP & $9(4)$ & $9(6)$ & $10(3)$ \\
\hline
\end{tabular}

Tabelle 1: Ausschnitt aus den in der Herzkatheteruntersuchung vor der partiellen cavopulmonalen Anastomose (PCPA) erhobenen hämodynamischen Daten der drei Altersgruppen: Als „frühzeitig“ wird ein Alter bei PCPA von kleiner gleich 90 Tagen (d), als ,elektiv“ zwischen 91 und 182 d und als „spät“ von über 182 d bezeichnet. Mittlerer pulmonalarterieller Druck (PAP), mittlerer linksatrialer Druck (LAP), mittlerer rechtsatrialer Druck (RAP), mittlerer transpulmonaler Gradient (TPG) und mittlerer enddiastolischer Ventrikeldruck (VEDP) jeweils in Millimeter-Quecksilbersäule $(\mathrm{mmHg})$. Mittelwert und Standardabweichung in Klammern. Es konnten keine signifikanten Unterschiede zwischen den Altersgruppen ermittelt werden. Das Signifikanzniveau lag bei p < $0,05$. Anzahl der Patienten pro Messparameter jeweils für die „frühzeitige“, die „elektive“ und die „späte“ Gruppe: PAP $\mathrm{n}=28, \mathrm{n}=94$ und $\mathrm{n}=28 ;$ LAP $\mathrm{n}=35, \mathrm{n}=114$ und $\mathrm{n}=33 ;$ RAP $\mathrm{n}=36, \mathrm{n}=119$ und $\mathrm{n}=36 ;$ TPG $\mathrm{n}=24, \mathrm{n}=86$ und $\mathrm{n}=25 ; \operatorname{VEDP} \mathrm{n}=38, \mathrm{n}=119$ und $\mathrm{n}=34$. 
Vor TCPA unterschieden sich weder der mittlere PAP, LAP noch der mittlere TPG zwischen den einzelnen Gruppen signifikant. Der VEDP stellte sich als signifikant höher bei den „Späten“ im Vergleich zu den „Elektiven“ dar ( $\mathrm{p}=0,049)$.

Hämodynamische Parameter vor Durchführung der TCPA im Vergleich zwischen den drei Altersgruppen

\begin{tabular}{|c|c|c|c|c|}
\hline & „frühzeitig“ & „elektiv“ & „spät““ & \\
\hline PAP & $9(3)$ & $9(2)$ & $9(2)$ & \\
\hline LAP & $6(3)$ & $5(2)$ & $5(2)$ & \\
\hline TPG & $4(3)$ & $4(1)$ & $4(1)$ & \\
\hline VEDP & $8(3)$ & $7(2)$ & $8(2)$ & $p=0,049$ \\
\hline
\end{tabular}

Tabelle 2: Auschnitt aus den in der Herzkatheteruntersuchung vor der totalen cavopulmonalen Anastomose (TCPA) erhobenen hämodynamischen Daten der drei Altersgruppen: Als „frühzeitig“wird ein Alter bei der partiellen cavopulmonalen Anastomose (PCPA) von kleiner gleich 90 Tagen (d), als ,elektiv“ zwischen 91 und 182 d und als „spät“ von über $182 \mathrm{~d}$ bezeichnet. Mittlerer pulmonalarterieller Druck (PAP), mittlerer linksatrialer Druck (LAP), mittlerer transpulmonaler Gradient (TPG) und mittlerer enddiastolischer Ventrikeldruck (VEDP) jeweils in $\mathrm{mmHg}$. Mittelwert und Standardabweichung in Klammern dahinter. Fett gedruckte Werte repräsentieren einen signfikanten Unterschied, der entsprechende p-Wert befindet sich in der rechten Spalte. Das Signifikanzniveau lag bei p < 0,05. Anzahl der Patienten pro Messparameter jeweils für die „frühzeitige“, die ,elektive“ und die „späte“ Gruppe: PAP $\mathrm{n}=25, \mathrm{n}=72$ und $\mathrm{n}=31 ;$ LAP $\mathrm{n}=24, \mathrm{n}=70$ und $\mathrm{n}=24 ;$ TPG $\mathrm{n}=24, \mathrm{n}=70$ und $\mathrm{n}=29 ;$ VEDP $\mathrm{n}=22, \mathrm{n}=65$ und $\mathrm{n}=29$.

\subsubsection{Größe und Wachstum der Pulmonalarterien}

Neben dem Vergleich der mittleren Pulmonalarterien-Indices zwischen den drei Altersgruppen erfolgte außerdem die statistische Gegenüberstellung der Indices zwischen Patienten mit einem SPShunt und Patienten mit einem RVPA-Shunt sowie zwischen Patienten mit HLHS und Patienten mit anderen Herzfehlern.

\subsubsection{Rechte Pulmonalarterie}

Patienten mit „frühzeitiger“ PCPA wiesen vor dieser Operation einen im Mittel signifikant kleineren Flächen-Index der rechten Pulmonalarterie im Vergleich zu der „elektiven“ $(p=0,004)$ und zu der „späten“ ( $\mathrm{p}=0,004)$ Altersgruppe auf. Die Mittelwerte der Patienten mit „elektiver“ und „später“ PCPA unterschieden sich nicht signifikant. Der Anteil der Patienten mit einer Stenose der rechten Pulmonalarterie lag bei 11,7 \% am gesamten Patientenkollektiv. Bezogen auf die drei Altersgruppen lag er bei $12,5 \%$ in der ,frühzeitigen“ Gruppe, bei 11,2\% in der ,elektiven“ und bei $12,8 \%$ in der „späten“ Gruppe und unterschied sich nicht signifikant zwischen den drei Altersgruppen. 
Es konnten keine signifikanten Unterschiede zwischen den drei Altersgruppen in den Mittelwerten der RPA-Flächen-Indices vor TCPA festgestellt werden.

Beim Vergleich der Pulmonalarterien-Indices vor PCPA und vor TCPA innerhalb der Altersgruppen zeigte sich, dass die Flächen-Indices der rechten Pulmonalarterie vor TCPA im Mittel signifikant größer waren als vor PCPA in der Gruppe der „Frühzeitigen“ $(p=0,026)$ und auch der „Elektiven“ ( $p$ = 0,026). Bei den Patienten mit ,später“ PCPA stellte sich kein signifikanter Unterschied dar.

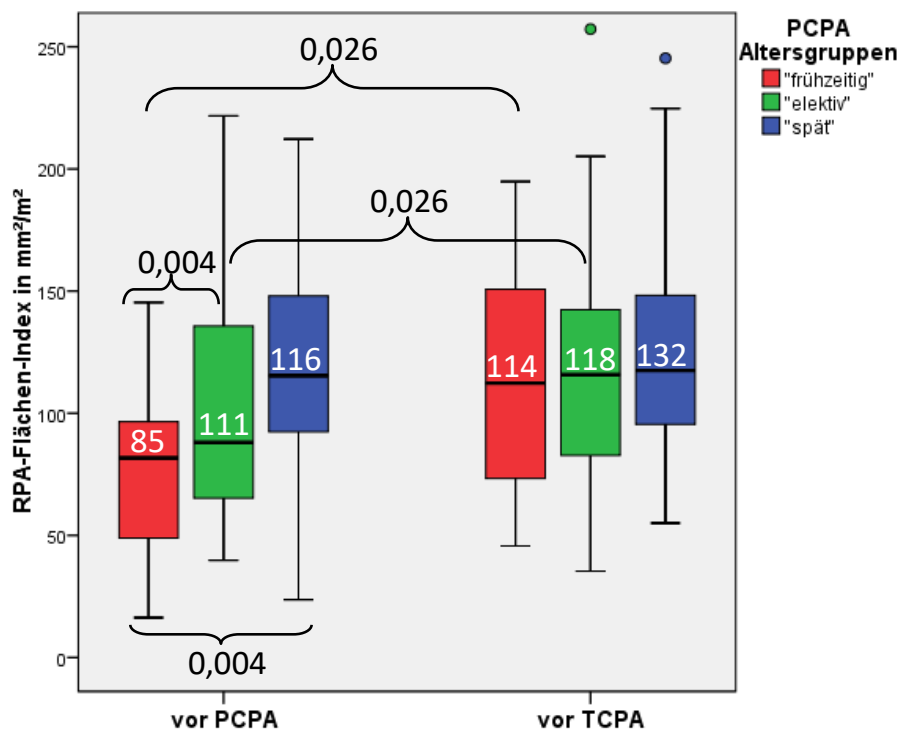

Abbildung 11: Vergleich der RPA-Flächen-Indices vor PCPA und vor TCPA zwischen und innerhalb der drei Altersgruppen

Vergleich der Flächen-Indices der rechten Pulmonalarterie (RPA) vor der partiellen cavopulmonalen Anastomose (PCPA) und vor der totalen cavopulmonalen Anastomose (TCPA) zwischen und innerhalb der drei Altersgruppen: Als „frühzeitig“ wird ein Alter bei PCPA von kleiner gleich 90 Tagen (d) (rot), als „elektiv“ zwischen 91 und 182 d (grün) und als „spät“ von über 182 d (blau) bezeichnet. Die weißen Zahlen in den Boxplots entsprechen dem mittleren RPA-Flächen-Index der jeweiligen Altersgruppe in Quadratmillimetern pro Quadratmeter $\left(\mathrm{mm}^{2} / \mathrm{m}^{2}\right)$. Signifikante Unterschiede zwischen zwei Gruppen sind durch eine Klammer gekennzeichnet, der entsprechende signifikante p-Wert steht darüber. Das Signifikanzniveau lag bei $\mathrm{p}<0,05$. Anzahl der Patienten vor PCPA in der „frühzeitigen“ Gruppe $n=40$, in der,,elektiven“ Gruppe n=124 und in der „späten“ Gruppe n = 36. Anzahl der Patienten vor TCPA in der „,frühzeitigen“ Gruppe $n=22$, in der ,elektiven“ Gruppe n = 74 und in der ,späten“ Gruppe $\mathrm{n}=27$.

\subsubsection{Linke Pulmonalarterie}

Die mittleren Flächen-Indices der linken Pulmonalarterie stellten sich vor PCPA bei den „Frühzeitigen“ als signifikant kleiner dar im Vergleich zu den „Elektiven“ ( $\mathrm{p}=0,018)$ sowie zu den „Späten“ ( $p=0,001)$. Auch die Patienten mit „elektiver“ PCPA wiesen im Mittel signifikant kleinere Flächen-Indices auf als die „späte“ Gruppe ( $\mathrm{p}=0,024)$. Der Anteil der Patienten mit einer Stenose der linken Pulmonalarterie vor PCPA lag bei 22,1\% am gesamten Patientenkollektiv. Bezogen auf die Altersgruppe lag er bei 22,5\% in der „frühzeitigen“ Gruppe, bei 23,9\% in der „elektiven“ und bei 15,4 $\%$ in der „späten“ Gruppe und unterschied sich nicht signifikant zwischen den drei Altersgruppen.

Patienten, die eine Stenose der linken Pulmonalarterie vor PCPA aufwiesen, zeigten sowohl vor PCPA $(\mathrm{p}<0,001)$ als auch vor TCPA $(\mathrm{p}=0,022)$ signifikant kleinere mittlere LPA-Flächen-Indices, als Patienten ohne diese Stenose. 
Vor TCPA zeigte sich, dass die mittleren LPA-Flächen-Indices der „Frühzeitigen“ signifikant kleiner waren als die der „Späten“ ( $p=0,001)$. Weder zwischen den Patienten mit ,frühzeitiger“ und „elektiver“ PCPA, noch zwischen der „elektiven“ und der „späten“ Gruppe zeigten sich signifikante Unterschiede in den Mittelwerten der LPA-Flächen-Indices.

Außerdem stellten sich die mittleren Flächen-Indices der linken Pulmonalarterie vor TCPA signifikant kleiner dar als vor PCPA in der „elektiven“ $(p=0,017)$ und in der „späten“ Gruppe $(p=0,005)$. Die mittleren LPA-Flächen-Indices der Patienten mit ,frühzeitiger“ PCPA wiesen keine signifikanten Unterschiede zwischen den beiden Zeitpunkten auf.

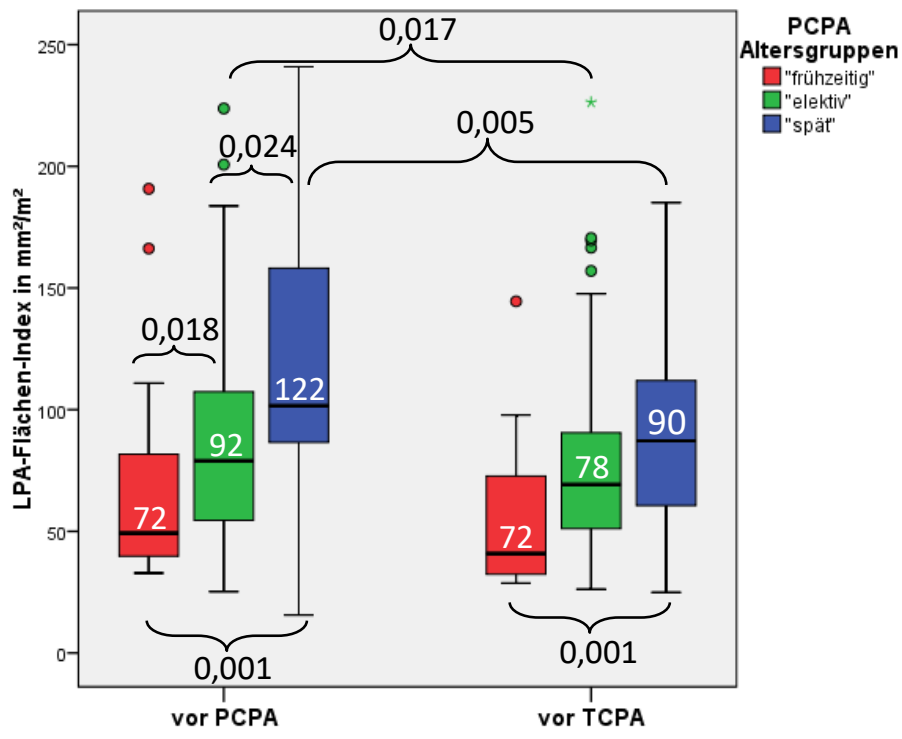

Abbildung 12: Vergleich der LPA-Flächen-Indices vor PCPA und vor TCPA zwischen und innerhalb der drei Altersgruppen

Vergleich der Flächen-Indices der linken Pulmonalarterie (LPA) vor der partiellen cavopulmonalen Anastomose (PCPA) und vor der totalen cavopulmonalen Anastomose (TCPA) zwischen und innerhalb der drei Altersgruppen: Als „frühzeitig“ wird ein Alter bei PCPA von kleiner gleich 90 Tagen (d) (rot), als „elektiv“ zwischen 91 und 182 d (grün) und als „spät“ von über 182 d (blau) bezeichnet. Die weißen Zahlen in den Boxplots entsprechen dem mittleren LPA-Flächen-Index der jeweiligen Altersgruppe in Quadratmillimetern pro Quadratmeter $\left(\mathrm{mm}^{2} / \mathrm{m}^{2}\right)$. Signifikante Unterschiede zwischen zwei Gruppen sind durch eine Klammer gekennzeichnet, der entsprechende signifikante p-Wert steht darüber. Das Signifikanzniveau lag bei $p<0,05$. Anzahl der Patienten vor PCPA in der „frühzeitigen“ Gruppe $n=40$, in der ,elektiven“ Gruppe n=124 und in der „,späten“ Gruppe n = 36. Anzahl der Patienten vor TCPA in der ,frühzeitigen“ Gruppe n = 22, in der,,elektiven“ Gruppe n $=74$ und in der ,späten“ Gruppe $n=27$.

\subsubsection{Nakata-Index}

Die mittleren Nakata-Indices verhielten sich vor PCPA ähnlich der RPA-Flächen-Indices. Es zeigten sich signifikant kleinerer mittlere Nakata-Indices in der ,frühzeitigen“ Gruppe im Vergleichen zu den „Elektiven“ ( $p=0,003)$ sowie zu den „Späten“ ( $p<0,001)$, jedoch kein signifikanter Unterschied zwischen der „elektiven“ und der „späten“ Gruppe.

Vor TCPA stellten sich die mittleren Nakata-Indices der Patienten mit „frühzeitiger“ PCPA als signifikant kleiner dar als die der Patienten mit „später“ PCPA $(p=0,008)$. Es konnten keine signifikanten Unterschiede zwischen den mittleren Nakata-Indices der „Frühzeitigen“ und der „Elektiven“ sowie zwischen den Werte der „Elektiven“ und der „Späten“ festgestellt werden. 
In der ,frühzeitigen“ Gruppe erwiesen sich die mittleren Nakata-Indices als signifikant größer vor TCPA als vor PCPA $(\mathrm{p}=0,042)$. In den anderen beiden Altersgruppen zeigten sich keine signifikanten Unterschiede zwischen den beiden Zeitpunkten.

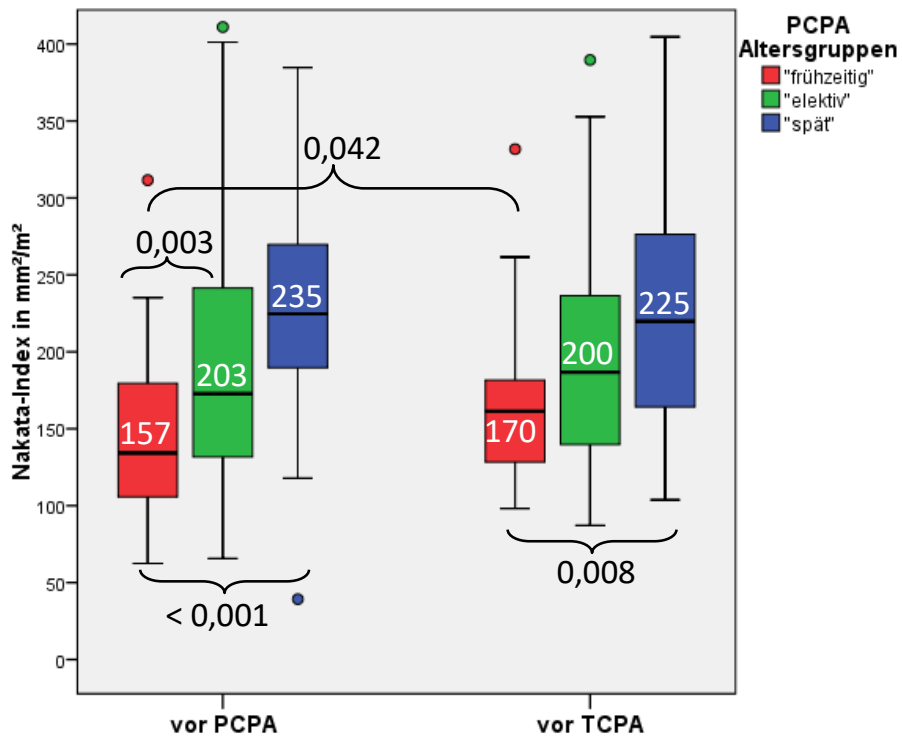

Abbildung 13: Vergleich der Nakata-Indices vor PCPA und vor TCPA zwischen und innerhalb der drei Altersgruppen

Vergleich der Nakata-Indices vor der partiellen cavopulmonalen Anastomose (PCPA) und vor der totalen cavopulmonalen Anastomose (TCPA) zwischen und innerhalb der drei Altersgruppen: Als "frühzeitig“ wird ein Alter bei PCPA von kleiner gleich 90 Tagen (d) (rot), als ,elektiv“ zwischen 91 und 182 d (grün) und als „,spät“ von über 182 d (blau) bezeichnet. Die weißen Zahlen in den Boxplots entsprechen dem mittleren Nakata-Index der jeweiligen Altersgruppe in Quadratmillimetern pro Quadratmeter $\left(\mathrm{mm}^{2} / \mathrm{m}^{2}\right)$. Signifikante Unterschiede zwischen zwei Gruppen sind durch eine Klammer gekennzeichnet, der entsprechende signifikante p-Wert steht darüber. Das Signifikanzniveau lag bei $\mathrm{p}<0,05$. Anzahl der Patienten vor PCPA in der ,frühzeitigen“ Gruppe $n=40$, in der „elektiven“ Gruppe n = 124 und in der „späten“ Gruppe n = 36. Anzahl der Patienten vor TCPA in der „frühzeitigen“ Gruppe $n=22$, in der „,elektiven“ Gruppe n $=74$ und in der „späten“ Gruppe $n=27$.

\subsubsection{Vergleich der Pulmonalarterien zwischen Patienten mit systemico-pulmonalen Shunt und Patienten mit right-ventricle-to-pulmonary-artery-Shunt}

Die Pulmonalarterien-Indices wurden neben dem Vergleich zwischen den drei Altersgruppen auch auf signifikante Unterschiede zwischen den verschiedenen Shuntarten untersucht. Hierbei wurden die Indices der Patienten mit RVPA-Shunt mit den Indices der Patienten mit SP-Shunt (zAP- und mBTShunt) verglichen.

Bei der Ausmessung der Pulmonalarterien vor PCPA konnte festgestellt werden, dass Patienten mit SP-Shunt signifikant größere mittlere LPA-Flächen-Indices $(\mathrm{p}<0,001)$ und Nakata-Indices $(\mathrm{p}<$ 0,001 ) aufwiesen als Patienten mit RVPA-Shunt. 


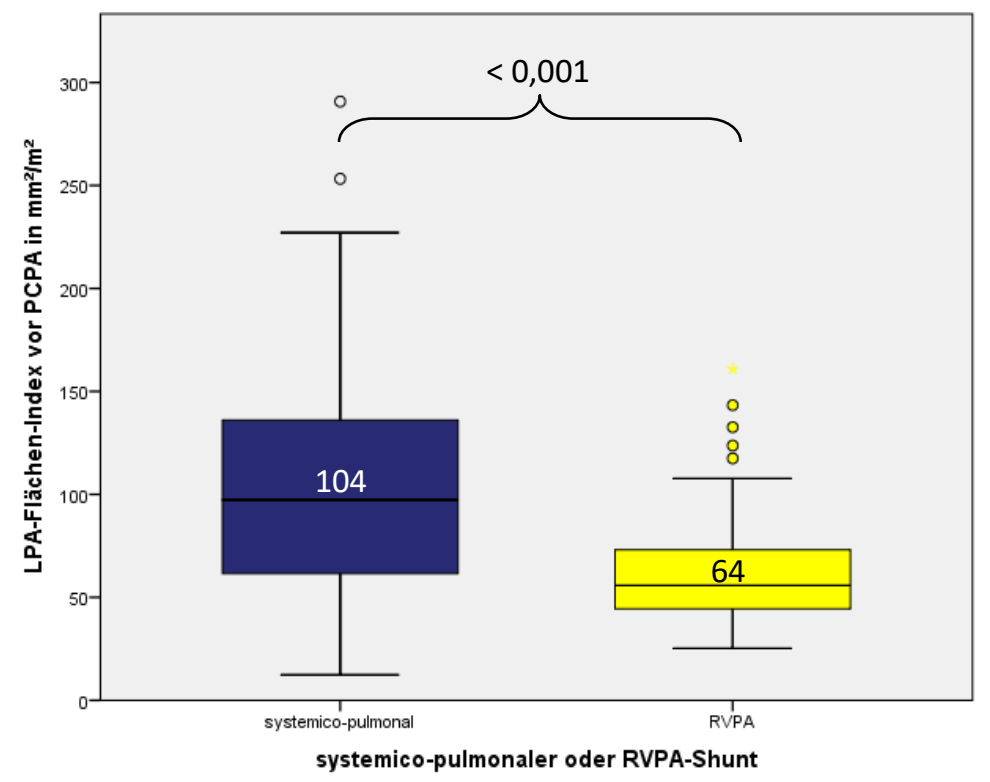

Abbildung 14: Vergleich der LPA-Flächen-Indices vor PCPA zwischen Patienten mit SP-Shunt und Patienten mit RVPA-Shunt

Vergleich der Flächen-Indices der linken Pulmonalarterie (LPA) vor der partiellen cavopulmonalen Anastomose (PCPA) zwischen Patienten mit systemico-pulmonalen Shunt (SP-Shunt: blau) und Patienten mit right-ventricle-topulmonary-artery-Shunt (RVPA-Shunt: gelb). Die weißen Zahlen in den Boxplots entsprechen dem mittleren LPAFlächen-Index der jeweiligen Shuntgruppe in Quadratmillimetern pro Quadratmeter $\left(\mathrm{mm}^{2} / \mathrm{m}^{2}\right)$. Signifikante Unterschiede zwischen zwei Gruppen sind durch eine Klammer gekennzeichnet, der entsprechende signifikante pWert steht darüber. Das Signifikanzniveau lag bei $\mathrm{p}<0,05$. Anzahl der Patienten mit SP-Shunt $\mathrm{n}=114$, Anzahl der Patienten mit RVPA-Shunt $n=63$.

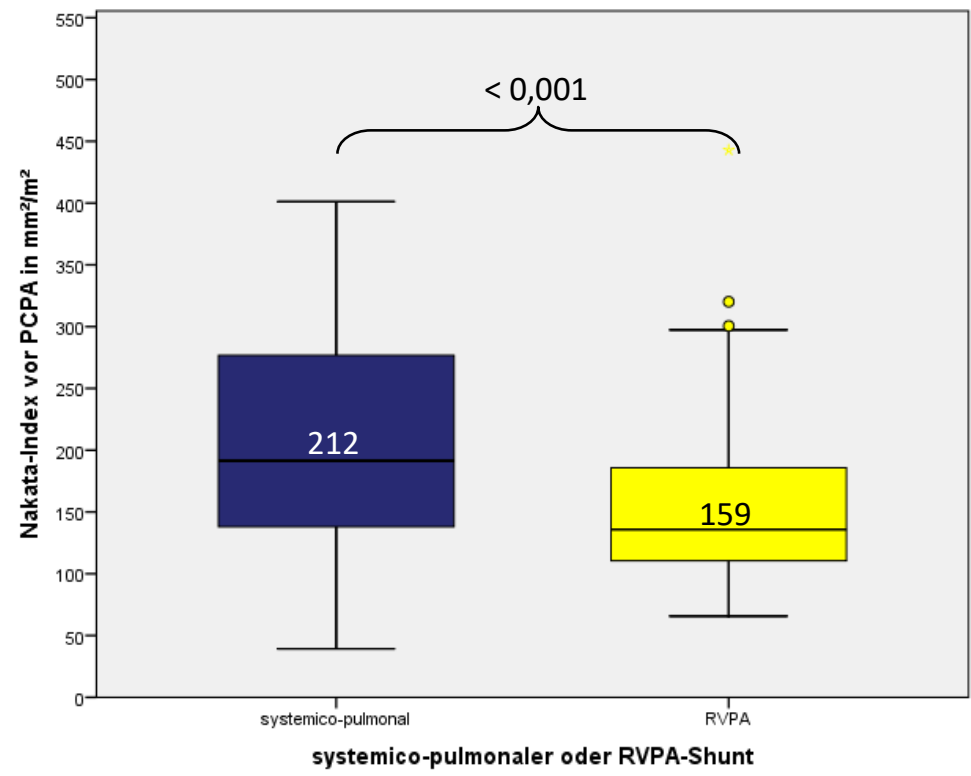

Abbildung 15: Vergleich der Nakata-Indices vor PCPA zwischen Patienten mit SP-Shunt und Patienten mit RVPAShunt

Vergleich der Nakata-Indices vor der partiellen cavopulmonalen Anastomose (PCPA) zwischen Patienten mit systemico-pulmonalen Shunt (SP-Shunt: blau) und Patienten mit right-ventricle-to-pulmonary-artery-Shunt (RVPA-Shunt: gelb). Die weißen Zahlen in den Boxplots entsprechen dem mittleren Nakata-Index der jeweiligen Shuntgruppe in Quadratmillimetern pro Quadratmeter $\left(\mathrm{mm}^{2} / \mathrm{m}^{2}\right)$. Signifikante Unterschiede zwischen zwei Gruppen sind durch eine Klammer gekennzeichnet, der entsprechende signifikante p-Wert steht darüber. Das

Signifikanzniveau lag bei $\mathrm{p}<0,05$. Anzahl der Patienten mit SP-Shunt $\mathrm{n}=114$, Anzahl der Patienten mit RVPAShunt $\mathrm{n}=63$. 
Die mittleren RPA-Flächen-Indices vor PCPA wiesen keine signifikanten Unterschiede zwischen den beiden Shuntgruppen auf.

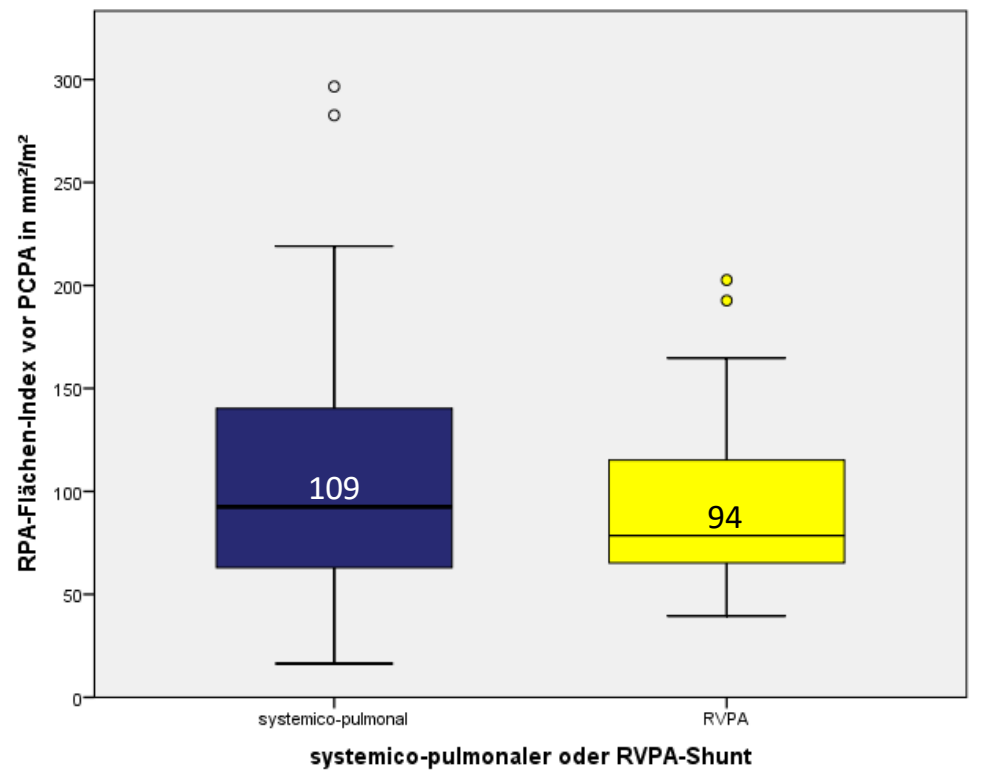

Abbildung 16: Vergleich der RPA-Flächen-Indices vor PCPA zwischen Patienten mit SP-Shunt und Patienten mit RVPA-Shunt

Vergleich der Flächen-Indices der rechten Pulmonalarterie (RPA) vor der partiellen cavopulmonalen Anastomose (PCPA) zwischen Patienten mit systemico-pulmonalen Shunt (SP-Shunt: blau) und Patienten mit right-ventricle-topulmonary-artery-Shunt (RVPA-Shunt: gelb). Die weißen Zahlen in den Boxplots entsprechen dem mittleren RPAFlächen-Index der jeweiligen Shuntgruppe in Quadratmillimetern pro Quadratmeter $\left(\mathrm{mm}^{2} / \mathrm{m}^{2}\right)$. Es konnten keine signifikanten Unterschiede zwischen den Shuntgruppen ermittelt werden. Das Signifikanzniveau lag bei $\mathrm{p}<0,05$. Anzahl der Patienten mit SP-Shunt $\mathrm{n}=114$, Anzahl der Patienten mit RVPA-Shunt $\mathrm{n}=63$.

Auch vor der Durchführung der TCPA konnten signifikant größere mittlere LPA-Flächen-Indices $(\mathrm{p}=$ $0,001)$ und Nakata-Indices $(p=0,029)$ bei Vorliegen eines SP-Shunts im Vergleich zum Vorliegen eines RVPA-Shunts festgestellt werden. 


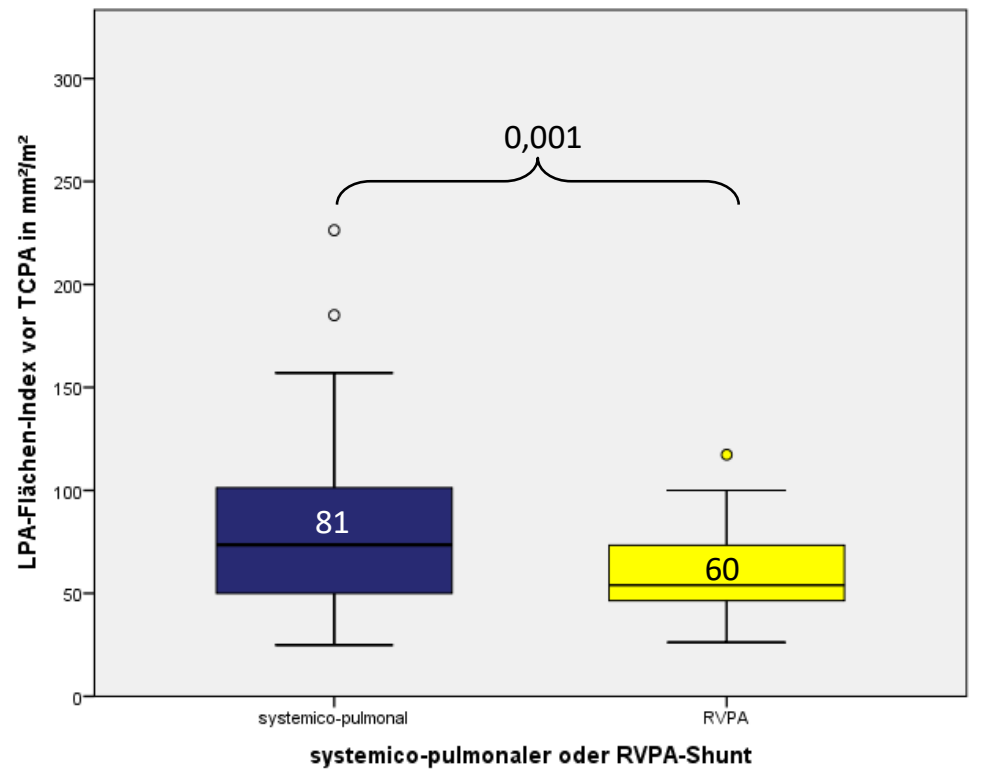

Abbildung 17: Vergleich der LPA-Flächen-Indices vor TCPA zwischen Patienten mit SP-Shunt und Patienten mit RVPA-Shunt

Vergleich der Flächen-Indices der linken Pulmonalarterie (LPA) vor der totalen cavopulmonalen Anastomose (TCPA) zwischen Patienten mit systemico-pulmonalen Shunt (SP-Shunt: blau) und Patienten mit right-ventricleto-pulmonary-artery-Shunt (RVPA-Shunt: gelb). Die weißen Zahlen in den Boxplots entsprechen dem mittleren LPA-Flächen-Index der jeweiligen Shuntgruppe in Quadratmillimetern pro Quadratmeter $\left(\mathrm{mm}^{2} / \mathrm{m}^{2}\right)$. Signifikante Unterschiede zwischen zwei Gruppen sind durch eine Klammer gekennzeichnet, der entsprechende signifikante p Wert steht darüber. Das Signifikanzniveau lag bei $\mathrm{p}<0,05$. Anzahl der Patienten mit SP-Shunt $\mathrm{n}=67$, Anzahl der Patienten mit RVPA-Shunt $\mathrm{n}=41$.

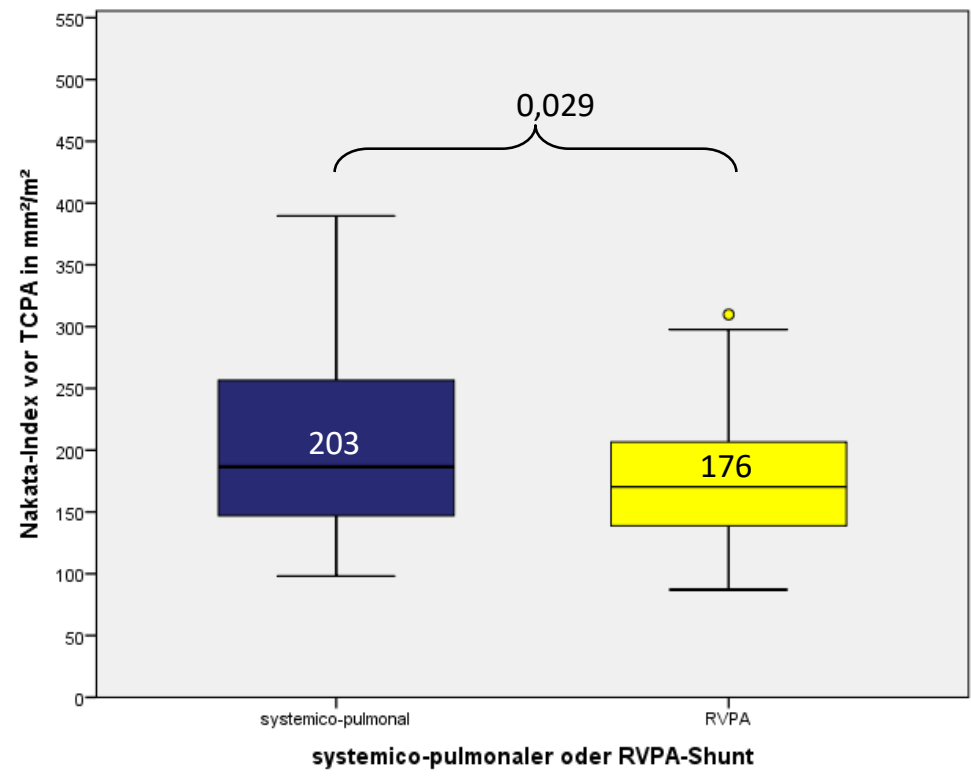

Abbildung 18: Vergleich der Nakata-Indices vor TCPA zwischen Patienten mit SP-Shunt und Patienten mit RVPAShunt

Vergleich der Nakata-Indices vor der totalen cavopulmonalen Anastomose (TCPA) zwischen Patienten mit systemico-pulmonalen Shunt (SP-Shunt: blau) und Patienten mit right-ventricle-to-pulmonary-artery-Shunt (RVPA-Shunt: gelb). Die weißen Zahlen in den Boxplots entsprechen dem mittleren Nakata-Index der jeweiligen Shuntgruppe in Quadratmillimetern pro Quadratmeter $\left(\mathrm{mm}^{2} / \mathrm{m}^{2}\right)$. Signifikante Unterschiede zwischen zwei Gruppen sind durch eine Klammer gekennzeichnet, der entsprechende signifikante p-Wert steht darüber. Das Signifikanzniveau lag bei $\mathrm{p}<0,05$. Anzahl der Patienten mit SP-Shunt $\mathrm{n}=67$, Anzahl der Patienten mit RVPAShunt $\mathrm{n}=41$. 
Die mittleren RPA-Flächen-Indices erwiesen sich auch vor TCPA als nicht signifikant unterschiedlich zwischen den beiden Shuntgruppen.

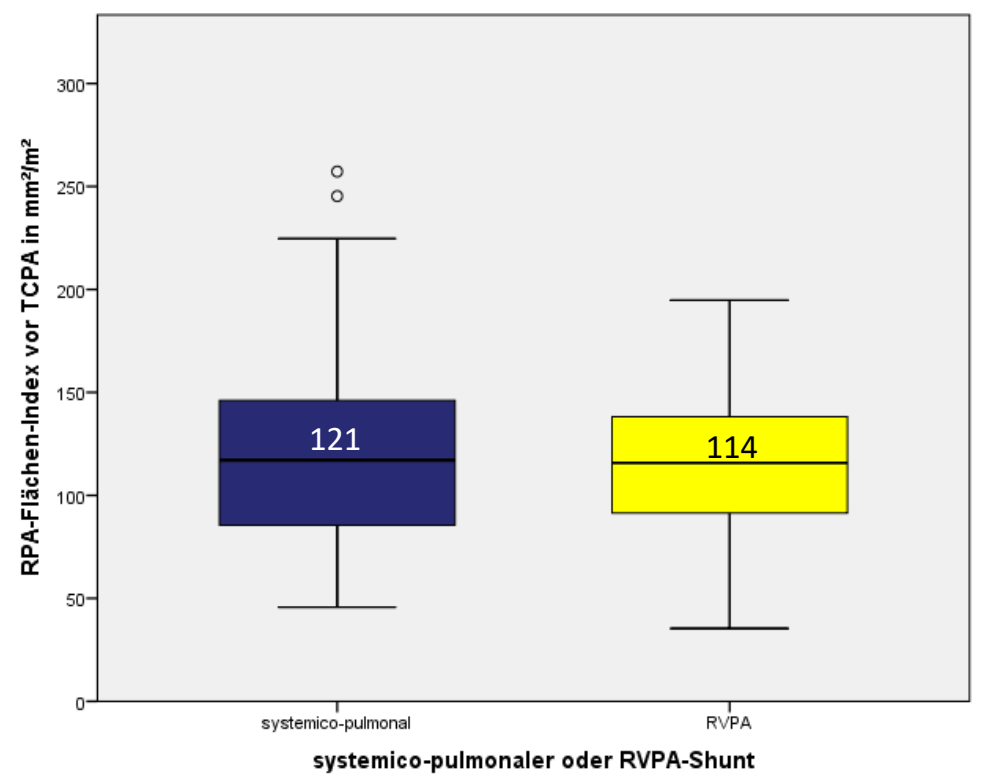

Abbildung 19: Vergleich der RPA-Flächen-Indices vor TCPA zwischen Patienten mit SP-Shunt und Patienten mit RVPA-Shunt

Vergleich der Flächen-Indices der rechten Pulmonalarterie (RPA) vor der totalen cavopulmonalen Anastomose (TCPA) zwischen Patienten mit systemico-pulmonalen Shunt (SP-Shunt: blau) und Patienten mit right-ventricleto-pulmonary-artery-Shunt (RVPA-Shunt: gelb). Die weißen Zahlen in den Boxplots entsprechen dem mittleren RPA-Flächen-Index der jeweiligen Shuntgruppe in Quadratmillimetern pro Quadratmeter $\left(\mathrm{mm}^{2} / \mathrm{m}^{2}\right)$. Es konnten keine signifikanten Unterschiede zwischen den Shuntgruppen ermittelt werden. Das Signifikanzniveau lag bei $\mathrm{p}<$ 0,05. Anzahl der Patienten mit SP-Shunt $n=67$, Anzahl der Patienten mit RVPA-Shunt $n=41$.

\subsubsection{Vergleich der Pulmonalarterien zwischen Patienten mit hypoplastischem Linksherzsyndrom und Patienten mit anderen Herzfehlern}

Der häufigste zu Grunde liegende Herzfehler im Patientenkollektiv war das HLHS mit 41,5\%. In der Gruppe der „Frühzeitigen“ lag der Anteil der Patienten mit HLHS bei 47,5\%, in der „elektiven“ Gruppe bei 48,2 \% und in der „späten“ Gruppe bei $12,5 \%$.

Im Vergleich der Altersgruppen konnte gezeigt werden, dass die mittleren Nakata-Indices und LPAFlächen-Indices der Patienten mit HLHS sowohl in der Gruppe der „Elektiven“ (LPA: $\mathrm{p}<0,001$; Nakata: $\mathrm{p}=0,036$ ) als auch der ,Späten“ (LPA: $\mathrm{p}<0,001$; Nakata: $\mathrm{p}<0,001$ ) vor PCPA signifikant kleiner waren als die Indices der Patienten mit anderen Herzfehlern.

In der ,frühzeitigen“ Gruppe waren nur die mittleren LPA-Flächen-Indices vor PCPA signifikant kleiner bei den Patienten mit HLHS als die der anderen Patienten $(p=0,018)$, nicht die mittleren Nakata-Indices. 


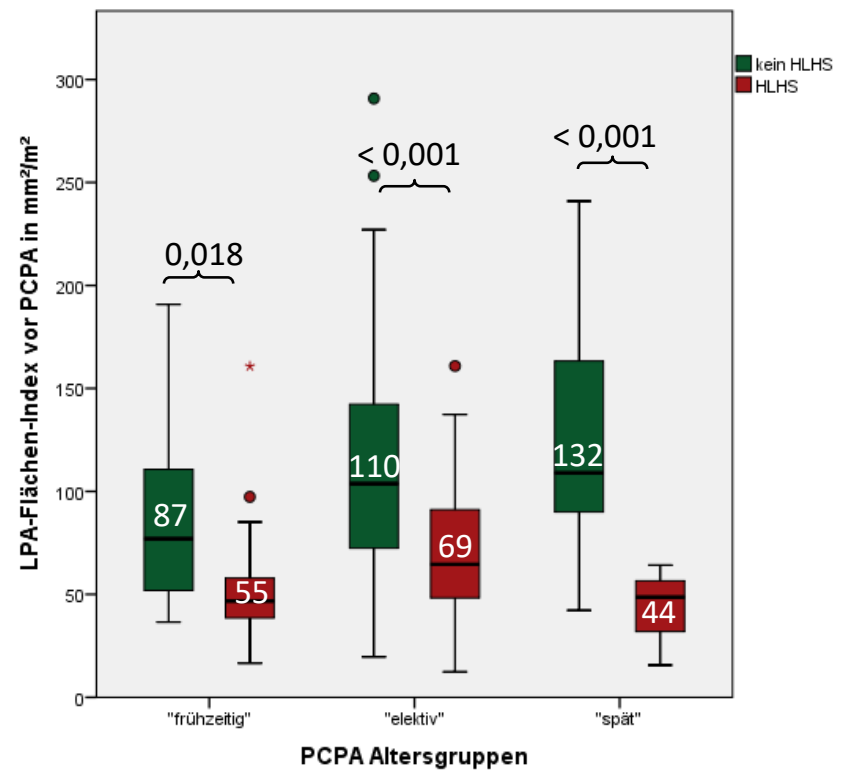

Abbildung 20: Vergleich der LPA-Flächen-Indices vor PCPA zwischen Patienten mit HLHS und Patienten mit anderen Herzfehlern

Vergleich der Flächen-Indices der linken Pulmonalarterie (LPA) vor der partiellen cavopulmonalen Anastomose (PCPA) zwischen Patienten mit hypoplastischen Linksherzsyndrom (HLHS: rot) und Patienten mit anderen Herzfehlern (kein HLHS: grün) innerhalb der drei Altersgruppen: Als „frühzeitig“ wird ein Alter bei PCPA von kleiner gleich 90 Tagen (d), als „elektiv“ zwischen 91 und 182 d und als „spät“ von über 182 d bezeichnet. Die weißen Zahlen in den Boxplots entsprechen dem mittleren LPA-Flächen-Index der jeweiligen Subgruppe in Quadratmillimetern pro Quadratmeter $\left(\mathrm{mm}^{2} / \mathrm{m}^{2}\right)$. Signifikante Unterschiede zwischen zwei Gruppen sind durch eine Klammer gekennzeichnet, der entsprechende signifikante p-Wert steht darüber. Das Signifikanzniveau lag bei $\mathrm{p}<0,05$. Anzahl der Patienten mit HLHS in der ,frühzeitigen“ Gruppe $\mathrm{n}=19$, in der ", elektiven“ Gruppe $\mathrm{n}=57$ und in der „späten“ Gruppe $n=4$. Anzahl der Patienten ohne HLHS in der „,frühzeitigen“ Gruppe $n=21$, in der „elektiven“ Gruppe $n=67$ und in der ,späten“ Gruppe $n=32$. 


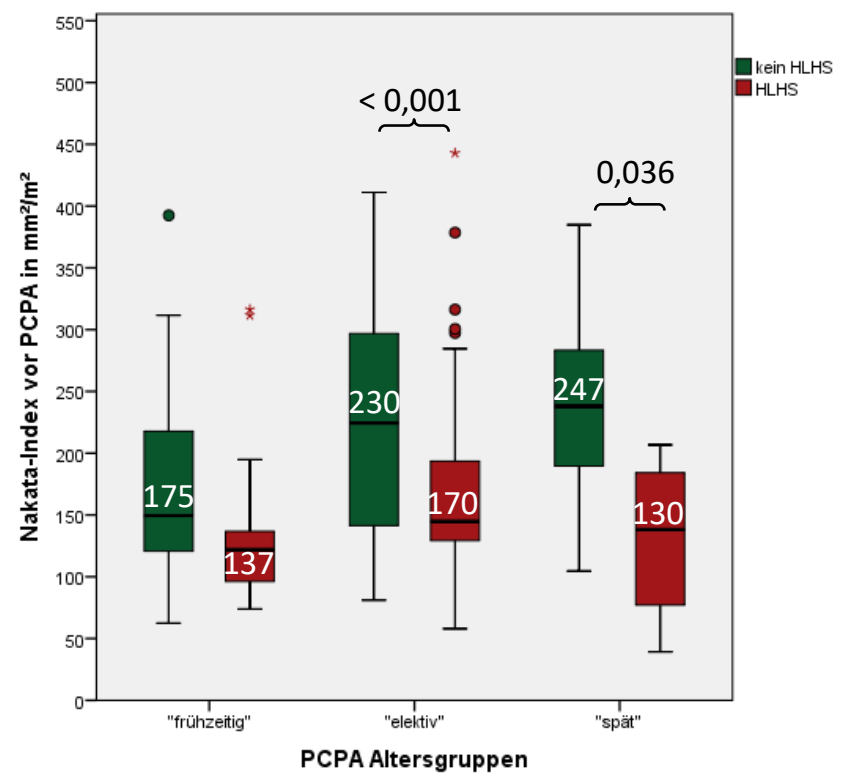

Abbildung 21: Vergleich der Nakata-Indices vor PCPA zwischen Patienten mit HLHS und Patienten mit anderen Herzfehlern

Vergleich der Nakata-Indices vor der partiellen cavopulmonalen Anastomose (PCPA) zwischen Patienten mit hypoplastischen Linksherzsyndrom (HLHS: rot) und Patienten mit anderen Herzfehlern (kein HLHS: grün) innerhalb der drei Altersgruppen: Als „frühzeitig“wird ein Alter bei PCPA von kleiner gleich 90 Tagen (d), als „elektiv“ zwischen 91 und 182 d und als „spät“ von über 182 d bezeichnet. Die weißen Zahlen in den Boxplots entsprechen dem mittleren Nakata-Index der jeweiligen Subgruppe in Quadratmillimetern pro Quadratmeter $\left(\mathrm{mm}^{2} / \mathrm{m}^{2}\right)$. Signifikante Unterschiede zwischen zwei Gruppen sind durch eine Klammer gekennzeichnet, der entsprechende signifikante p-Wert steht darüber. Das Signifikanzniveau lag bei p < 0,05. Anzahl der Patienten mit HLHS in der „frühzeitigen“ Gruppe $n=19$, in der ,elektiven“ Gruppe $n=57$ und in der ,späten“ Gruppe n = 4. Anzahl der Patienten ohne HLHS in der ,frühzeitigen“ Gruppe $n=21$, in der ,elektiven“ Gruppe $n=67$ und in der „späten“ Gruppe n $=32$.

Vor TCPA zeigten sich in der Gruppe der „Elektiven“ die mittleren LPA-Flächen-Indices und NakataIndices wieder als signifikant kleiner bei den Patienten mit HLHS als beim restlichen Kollektiv (LPA: $p=0,002 ;$ Nakata: $p=0,026)$.

In der „frühzeitigen“ Gruppe waren nur die mittleren LPA-Flächen-Indices der Patienten mit HLHS signifikant kleiner als die der Patienten mit anderen Herzfehlern $(\mathrm{p}=0,002)$. 


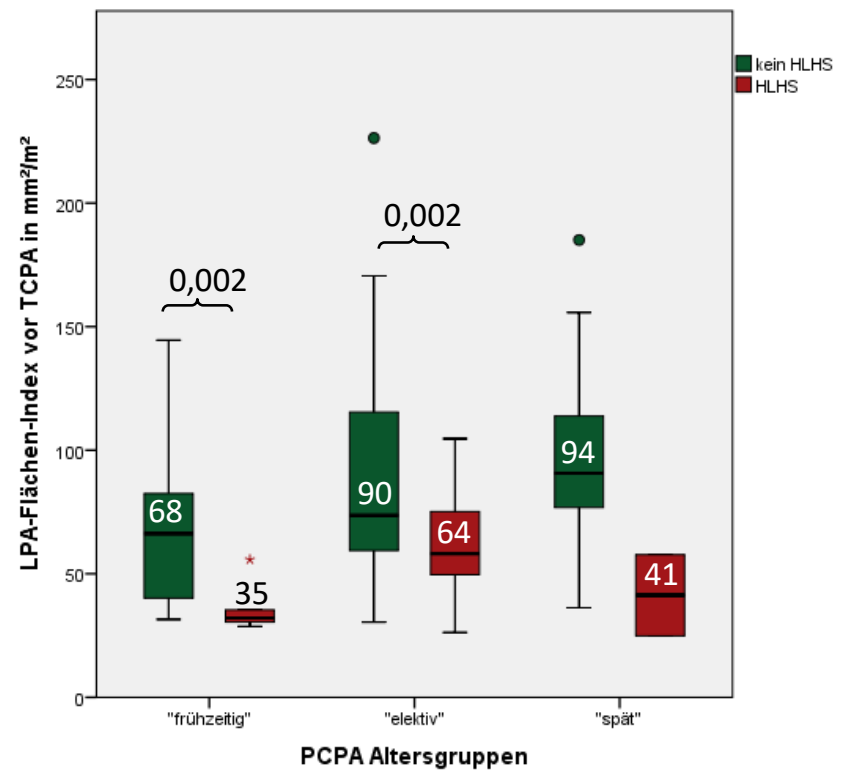

Abbildung 22: Vergleich der LPA-Flächen-Indices vor TCPA zwischen Patienten mit HLHS und Patienten mit anderen Herzfehlern

Vergleich der Flächen-Indices der linken Pulmonalarterie (LPA) vor der totalen cavopulmonalen Anastomose (TCPA) zwischen Patienten mit hypoplastischen Linksherzsyndrom (HLHS: rot) und Patienten mit anderen Herzfehlern (kein HLHS: grün) innerhalb der drei Altersgruppen: Als „frühzeitig“ wird ein Alter bei der partiellen cavopulmonalen Anastomose (PCPA) von kleiner gleich 90 Tagen (d), als „elektiv“ zwischen 91 und 182 d und als „spät“ von über 182 d bezeichnet. Die weißen (bzw. schwarze) Zahlen in den Boxplots (bzw. über dem roten Boxplot in der „frühzeitigen“ Gruppe) entsprechen dem mittleren LPA-Flächen-Index der jeweiligen Subgruppe in Quadratmillimetern pro Quadratmeter $\left(\mathrm{mm}^{2} / \mathrm{m}^{2}\right)$. Signifikante Unterschiede zwischen zwei Gruppen sind durch eine Klammer gekennzeichnet, der entsprechende signifikante p-Wert steht darüber. Das Signifikanzniveau lag bei $\mathrm{p}<0,05$. Anzahl der Patienten mit HLHS in der „, frühzeitigen“ Gruppe $n=8$, in der „,elektiven“ Gruppe $n=35$ und in der ,späten“ Gruppe $n=2$. Anzahl der Patienten ohne HLHS in der ,frühzeitigen“ Gruppe n = 14, in der „elektiven“ Gruppe n = 39 und in der ,späten“ Gruppe n = 25 . 


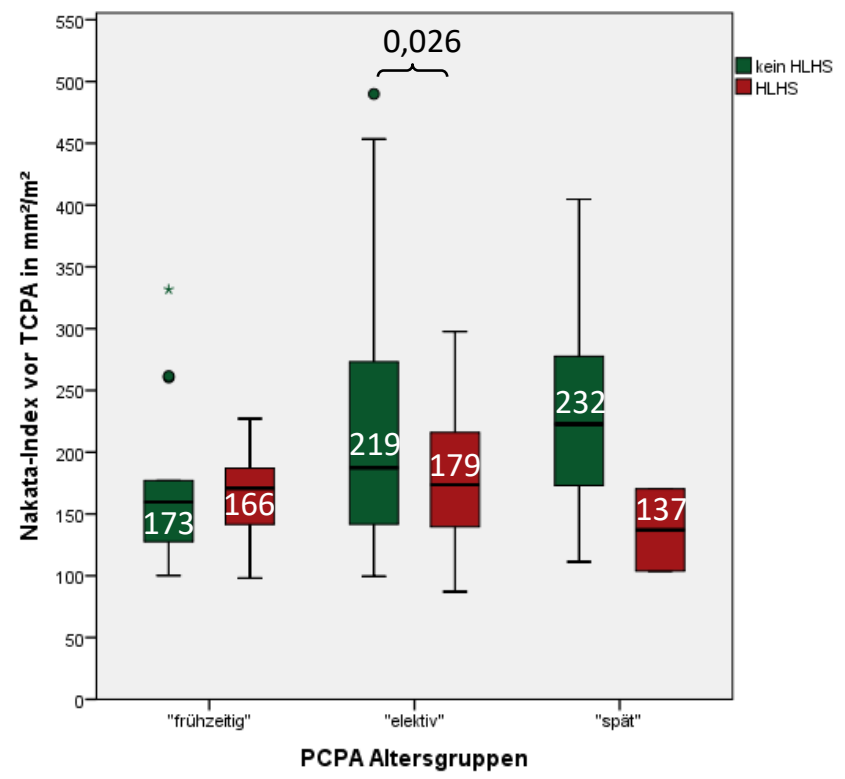

Abbildung 23: Vergleich der Nakata-Indices vor TCPA zwischen Patienten mit HLHS und Patienten mit anderen Herzfehlern

Vergleich der Nakata-Indices vor der totalen cavopulmonalen Anastomose (TCPA) zwischen Patienten mit hypoplastischen Linksherzsyndrom (HLHS: rot) und Patienten mit anderen Herzfehlern (kein HLHS: grün) innerhalb der drei Altersgruppen: Als ,frühzeitig“ wird ein Alter bei der partiellen cavopulmonalen Anastomose (PCPA) von kleiner gleich 90 Tagen (d), als „elektiv“ zwischen 91 und 182 d und als „spät“ von über 182 d bezeichnet. Die weißen Zahlen in den Boxplots entsprechen dem mittleren Nakata-Index der jeweiligen Subgruppe in Quadratmillimetern pro Quadratmeter $\left(\mathrm{mm}^{2} / \mathrm{m}^{2}\right)$. Signifikante Unterschiede zwischen zwei Gruppen sind durch eine Klammer gekennzeichnet, der entsprechende signifikante p-Wert steht darüber. Das Signifikanzniveau lag bei $\mathrm{p}<0,05$. Anzahl der Patienten mit HLHS in der ,frühzeitigen“ Gruppe $\mathrm{n}=8$, in der „elektiven“ Gruppe $\mathrm{n}=35$ und in der „späten“ Gruppe $n=2$. Anzahl der Patienten ohne HLHS in der „,frühzeitigen“ Gruppe $n=14$, in der „elektiven“ Gruppe $n=34$ und in der ,späten“ Gruppe n $=25$.

Die mittleren RPA-Flächen-Indices unterschieden sich zwischen Patienten mit HLHS und Patienten mit anderen Herzfehlern weder vor PCPA noch vor TCPA signifikant.

\subsubsection{Einflussfaktoren auf die Größe der Pulmonalarterien}

Das Alter zum Zeitpunkt der PCPA zeigte sich als signifikant positiver Einflussfaktor auf die vor der PCPA gemessenen LPA-Flächen-Indices ( $\mathrm{p}=0,001 ; \mathrm{B}=0,160)$, RPA-Flächen-Indices ( $\mathrm{p}=0,026$; $\mathrm{B}$ $=0,115)$ und Nakata-Indices $(\mathrm{p}=0,002 ; \mathrm{B}=0,260)$.

Dieser signifikante, positive Zusammenhang konnte auch für die vor TCPA gemessenen Pulmonalarterien-Indices nachgewiesen werden (LPA: $p=0,001 ; B=0,168 ; R P A: p=0,030 ; B=$ 0,145; Nakata: $\mathrm{p}=0,004 ; \mathrm{B}=0,309$ ).

Auch das Körpergewicht vor PCPA korrelierte signifikant positiv mit den Pulmonalarterien-Indices, die vor PCPA (LPA: $p<0,001 ; B=0,016$; RPA: $p=0,006$; $=0,009$; Nakata: $p<0,001 ; B=0,025$ ) und auch die vor TCPA gemessen wurden (LPA: $p=0,001 ; \mathrm{B}=0,009 ;$ RPA: $p=0,005 ; \mathrm{B}=0,010$; Nakata: $\mathrm{p}<0,001 ; \mathrm{B}=0,021$ ).

Eine Shuntstenose vor PCPA stellte sich als negativen Einflussfaktor auf den Flächen-Index der LPA $(\mathrm{p}<0,001 ; \mathrm{B}=-39,572)$ und auf den Nakata-Index vor PCPA $(\mathrm{p}<0,001 ; \mathrm{B}=-52,470)$ dar.

Das Vorhandensein einer stenosierten Pulmonalarterie vor PCPA korrelierte negativ mit dem FlächenIndex der jeweiligen Seite, der vor PCPA gemessen wurde (LPA: $p<0,001 ; B=-41,826$; RPA: $p=$ 
0,011; B = -31,057). Wiesen eine oder beide Pulmonalarterien eine Stenose auf, korrelierte dies negativ mit dem Nakata-Index vor PCPA ( $<<0,001 ; B=-54,619)$.

Eine Stenose der jeweiligen Pulmonalarterie vor TCPA hatte keinen signifikanten Einfluss auf die Größe der vor TCPA gemessenen Pulmonalarterien-Indices. Auch der Nakata-Index vor TCPA korrelierte nicht mit dem Vorhandensein einer Stenose einer oder beider Pulmonalarterien vor TCPA.

Das Vorhandensein eines RVPA-Shunts zeigte sich als signifikant negativer Einflussfaktor auf den LPA-Flächen-Index und Nakata-Index, sowohl vor PCPA ( LPA: $p<0,001 ; B=-39,466$; Nakata: $p<$ 0,$001 ; \mathrm{B}=-53,616$ ) als auch vor TCPA (LPA: $\mathrm{p}=0,003 ; \mathrm{B}=-21,296$; Nakata: $\mathrm{p}=0,046 ; \mathrm{B}=-$ 26,842). Der RPA-Flächen-Index wurde weder vor PCPA noch vor TCPA von der Shuntart beeinflusst.

Patienten mit RVPA-Shunt wiesen signifikant häufiger eine Shuntstenose $(\mathrm{p}<0,001)$ sowie eine Stenose der linken $(p=0,010)$ und/oder beider Pulmonalarterien $(p=0,023)$ vor PCPA auf, als Patienten mit einem SP-Shunt.

Die Gewichtszunahme zwischen PCPA und TCPA konnte nicht als Einflussfaktor auf die vor TCPA gemessenen Pulmonalarterien-Indices detektiert werden.

\subsection{Perioperativer Verlauf: Dauer des Kreislaufstillstandes mittels Herz-Lungen-Maschine}

Die Dauer des Kreislaufstillstandes während der Durchführung der PCPA mittels Herz-LungenMaschine lag im Median in der ,frühzeitigen“ Gruppe bei 63 Minuten (min) (Minium 27 min, Maximum 140 min), in der „elektiven“ Gruppe bei 61 min (Minimum 24 min, Maximum 193 min) und in der ,späten“ Gruppe bei 58 min (Minimum 20 min, Maximum 138 min). Diese Ergebnisse unterschieden sich zwischen den Altersgruppen nicht signifikant.

\subsection{Postoperativer Verlauf nach Durchführung der partiellen cavopulmonalen Anastomose}

\subsubsection{Beatmungsdauer, arterielle Sauerstoffsättigung, Sauerstoffbedarf und transkutane Sauerstoffsättigung bei Entlassung}

Im postoperativen Verlauf nach PCPA wurden die „Späten“ im Median 8 Stunden nachbeatmet, die „Frühzeitigen“ mit 15 Stunden fast doppelt so lang. Dieser Unterschied zeigte sich als signifikant ( $\mathrm{p}=$ 0,039). Die „Elektiven“ wurden im Median 10 Stunden nachbeatmet und unterschieden sich somit weder signifikant von den „Frühzeitigen“ noch von den „Späten“. 


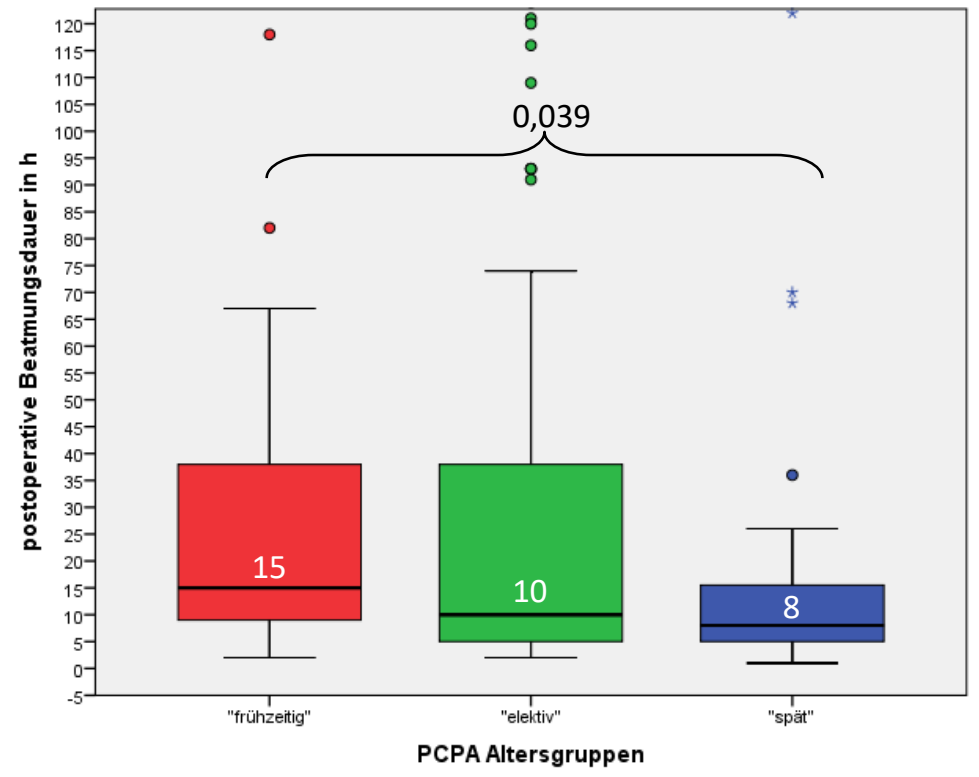

Abbildung 24: Vergleich der postoperativen Beatmungsdauer nach PCPA zwischen den drei Altersgruppen

Vergleich der postoperativen Beatmungsdauer in Stunden (h) nach der partiellen cavopulmonalen Anastomose (PCPA) zwischen den drei Altersgruppen: Als „frühzeitig“wird ein Alter bei PCPA von kleiner gleich 90 Tagen (d) (rot), als ,elektiv“ zwischen 91 und 182 d (grün) und als ,spät“ von über 182 d (blau) bezeichnet. Die weißen Zahlen in den Boxplots entsprechen der medianen Beatmungsdauer der jeweiligen Altersgruppe in Stunden (h). Signifikante Unterschiede zwischen zwei Gruppen sind durch eine Klammer gekennzeichnet, der entsprechende signifikante $\mathrm{p}$-Wert steht darüber. Das Signifikanzniveau lag bei $\mathrm{p}<0,05$. Anzahl der Patienten in der „frühzeitigen“ Gruppe n = 37, in der „elektiven“ Gruppe n = 126 und in der „späten“ Gruppe n = 39 .

In der über $24 \mathrm{~h}$ gemittelten arteriellen Sauerstoffsättigung $\left(\mathrm{SaO}_{2}\right)$ konnte $72 \mathrm{~h}$ nach PCPA kein signifikanter Unterschied zwischen den drei Altersgruppen festgestellt werden. 48 Stunden postoperativ zeigten die „Späten“ signifikant höhere $\mathrm{SaO}_{2}$-Werte als die „Frühzeitigen“ ( $\left.\mathrm{p}=0,038\right)$ und als die „Elektiven“ ( $p=0,034)$. Letztere wiesen zu diesem Zeitpunkt keine signifikant höheren $\mathrm{SaO}_{2}$-Werte auf als die „Frühzeitigen“. In den ersten 24 h postoperativ wies die „späte“ Gruppe signifikant höherer $\mathrm{SaO}_{2}$-Werte auf als die ,frühzeitige“ ( $\left.\mathrm{p}<0,001\right)$ und die ,elektive“ $(\mathrm{p}<0,001)$ Gruppe. Auch die „Elektiven“ zeigten signifikant höhere Werte als die „Frühzeitigen“ ( $p=0,022)$. 


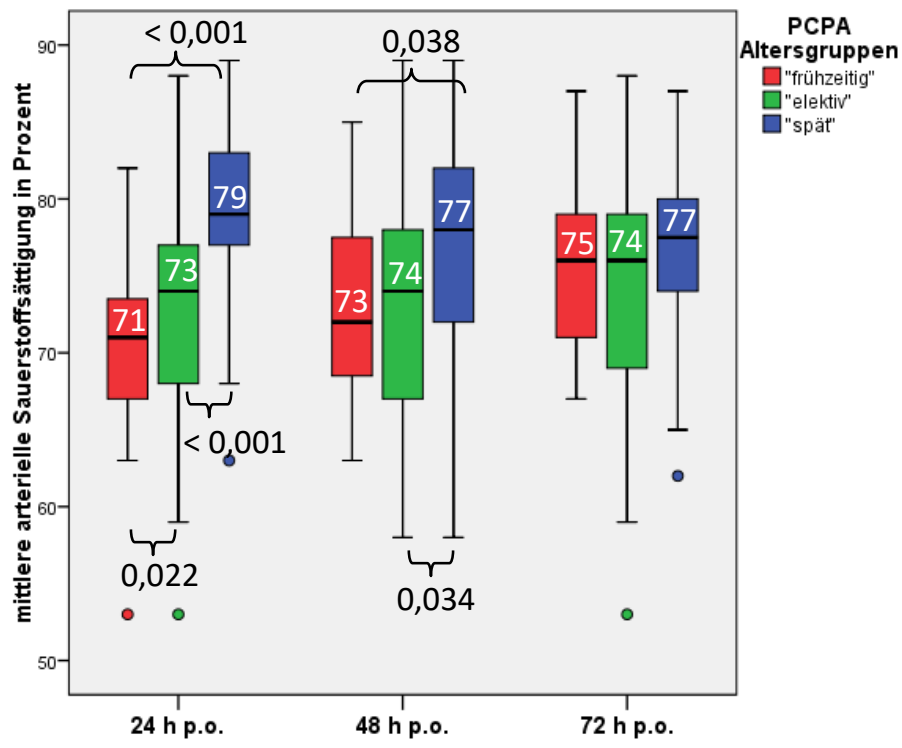

Abbildung 25: Vergleich der postoperativen mittleren arteriellen Sauerstoffsättigung nach PCPA zwischen den drei Altersgruppen

Vergleich der postoperativen mittleren arteriellen Sauerstoffsättigung $\left(\mathrm{SaO}_{2}\right)$ in Prozent (\%) gemittelt über 24 Stunden (h), $48 \mathrm{~h}$ und $72 \mathrm{~h}$ postoperativ (p.o.) nach der partiellen cavopulmonalen Anastomose (PCPA) zwischen den drei Altersgruppen: Als „frühzeitig“ wird ein Alter bei PCPA von kleiner gleich 90 Tagen (d) (rot), als „elektiv“ zwischen 91 und $182 \mathrm{~d}$ (grün) und als „spät“ von über $182 \mathrm{~d}$ (blau) bezeichnet. Die weißen Zahlen in den Boxplots entsprechen der mittleren $\mathrm{SaO}_{2}$ der jeweiligen Altersgruppe in Prozent (\%). Signifikante Unterschiede zwischen zwei Gruppen sind durch eine Klammer gekennzeichnet, der entsprechende signifikante p-Wert steht darüber. Das Signifikanzniveau lag bei p < 0,05. Anzahl der Patienten pro Messparameter jeweils für die „frühzeitige“, die „elektive“ und die „späte“ Gruppe: $\mathrm{SaO}_{2} 24$ h p.o. n = 37, n = 127 und n = 39 ; $\mathrm{SaO} 248$ h p.o. n $=36, \mathrm{n}=125$ und $\mathrm{n}=36 ; \mathrm{SaO}_{2} 72 \mathrm{~h}$ p.o. $\mathrm{n}=32, \mathrm{n}=102$ und $\mathrm{n}=23$.

Einen postoperativen Sauerstoffbedarf über sieben Tage wiesen 51,4\% der Patienten mit „frühzeitiger“, 59\% mit „elektiver“ und 35,9\% mit „später“ PCPA auf. Diese Ergebnisse unterschieden sich nicht signifikant.

Die transkutane Sauerstoffsättigung der Patienten lag bei Entlassung aus dem Krankenhaus nach Durchführung der PCPA im Mittel bei 80,3\% in der „frühzeitigen“, bei 81,3\% in der „elektiven“ und bei $81,4 \%$ in der „späten“ Gruppe. Die Ergebnisse unterschieden sich nicht signifikant voneinander.

\subsubsection{Pulmonalarterieller Druck und transpulmonaler Gradient}

Patienten mit „frühzeitiger“ PCPA wiesen weder 24 h, 48 h noch 72 h postoperativ einen signifikant höheren mittleren pulmonalarteriellen Druck (PAP) auf als Patienten mit „elektiver“ PCPA. Dies erwies sich auch im Vergleich der „elektiven“ mit der „,späten“ Altersgruppe. Der mittlere PAP der „Späten“ war 48 h postoperativ signifikant niedriger als bei den „Frühzeitigen“ ( $p=0,034), 24$ h zuvor bzw. danach bestand dieser Unterschied nicht mehr.

Bei der Auswertung des postoperativen mittleren transpulmonalen Gradienten (TPG) über 24 bzw. 48 h konnten zu keinem Zeitpunkt signifikante Unterschiede zwischen den drei Altersgruppen festgestellt werden. 


\section{Postoperativer mittlerer pulmonalarterieller Druck und transpulmonaler Gradient im Vergleich zwischen den drei Altersgruppen}

\begin{tabular}{|c|c|c|c|c|}
\hline & „frühzeitig“ & „elektiv“ & „spät““ & \\
\hline PAP $24 \mathrm{~h}$ & $16(2)$ & $16(3)$ & $15(3)$ & \\
\hline PAP 48 h & $16(2)$ & $15(3)$ & $14(3)$ & $p=0,034$ \\
\hline PAP $72 \mathrm{~h}$ & $13(2)$ & $14(4)$ & $14(3)$ & \\
\hline TPG $24 \mathrm{~h}$ & 7 (3) & $8(3)$ & $7(2)$ & \\
\hline TPG $48 \mathrm{~h}$ & 7 (2) & $7(3)$ & $7(4)$ & \\
\hline
\end{tabular}

Tabelle 3: Hämodynamische Parameter im postoperativen Verlauf nach Durchführung der partiellen cavopulmonalen Anastomose (PCPA) in den drei Altersgruppen: Als „frühzeitig“wird ein Alter bei PCPA von kleiner gleich 90 Tagen (d), als „elektiv“ zwischen 91 und $182 \mathrm{~d}$ und als „spät“ von über $182 \mathrm{~d}$ bezeichnet. Mittlerer pulmonalarterieller Druck (PAP) und mittlerer transpulmonaler Gradient (TPG) jeweils in MillimeterQuecksilbersäule $(\mathrm{mmHg})$, gemittelt über 24 Stunden $(\mathrm{h}), 48 \mathrm{~h}$ und $72 \mathrm{~h}$ postoperativ. Mittelwert und Standardabweichung in Klammern. Fett gedruckte Werte repräsentieren einen signfikanten Unterschied, der entsprechende $\mathrm{p}$-Wert befindet sich in der rechten Spalte. Das Signifikanzniveau lag bei $\mathrm{p}<0,05$. Anzahl der Patienten pro Messparameter jeweils für die „frühzeitige“, die „elektive“ und die „späte“ Gruppe: PAP 24 h n = $36, \mathrm{n}=121$ und $\mathrm{n}=36$; PAP $48 \mathrm{~h} \mathrm{n}=22, \mathrm{n}=97$ und $\mathrm{n}=26$; PAP $72 \mathrm{~h} \mathrm{n}=14, \mathrm{n}=45$ und $\mathrm{n}=9$; TPG $24 \mathrm{~h} \mathrm{n}=$ $27, \mathrm{n}=101$ und $\mathrm{n}=30 ;$ TPG $48 \mathrm{~h} \mathrm{n}=14, \mathrm{n}=58$ und $\mathrm{n}=9$.

\subsubsection{Dauer des intensivmedizinischen und stationären Aufenthaltes}

Die mediane Aufenthaltsdauer der Patienten auf der Intensivstation nach PCPA lag in der „frühzeitigen“ Gruppe bei 8 Tagen (Minimum 2 d, Maximum 54 d), in der „elektiven“ Gruppe bei 7 Tagen (Minimum 2 d, Maximum 41 d), und in der „späten“ Gruppe bei 4 Tagen (Minimum $1 \mathrm{~d}$, Maximum $21 \mathrm{~d})$.

Als signifikant erwies sich hierbei die kürzere mediane Verweildauer auf der Intensivstation der „Späten“ im Vergleich zu der „frühzeitigen“ ( $p=0,001)$ und der „elektiven“ Gruppe ( $p=0,003)$. Die „Frühzeitigen“ befanden sich nicht signifikant länger auf der Intensivstation als die „Elektiven“.

Die mediane Dauer des gesamten stationären Aufenthaltes der Patienten nach PCPA lag in der „frühzeitigen“ Gruppe bei 19 Tagen (Minimum 7 d, Maximum 161 d), in der „elektiven“ Gruppe bei 15 Tagen (Minimum 2 d, Maximum 51 d), und in der „späten“ Gruppe bei 14 Tagen (Minimum 5 d, Maximum $35 \mathrm{~d})$.

Patienten mit „elektiver“ PCPA befanden sich im Median nicht signifikant länger im Krankenhaus als Patienten mit „später“ PCPA. Die Aufenthaltsdauer der Patienten mit „frühzeitiger“ PCPA erwies sich als signifikant länger im Vergleich zu der „elektiven“ $(p=0,024)$ und der „späten“ Gruppe $(p=0,003)$. 


\section{5 Überleben}

\subsubsection{Postoperative Mortalität und Gesamtmortalität}

Die Gesamtmortalität im Patientenkollektiv lag bei 9,9\% bei einer medianen Beobachtungszeit von zwei Jahren (Minimum 1 d, Maximum 8,3 Jahre). 3,4 \% der Patienten verstarben innerhalb der ersten 30 Tage nach PCPA und 6,5\% der Patienten nach diesem Zeitraum bis zum Ende des

Nachbeobachtungszeitraums. Bezogen auf die drei Altersgruppen lag die Gesamtmortalität bei 5,3\% in der „frühzeitigen“, bei 12,6 \% in der „elektiven“ und bei 5,3\% in der „späten“ Gruppe. Todesfälle innerhalb der ersten 30 Tage nach PCPA konnten nur in der „elektiven“ Gruppe beobachtet werden. Es zeigten sich keine signifikanten Unterschiede in der 30-Tage-Mortalität oder in der Gesamtmortalität zwischen den drei Altersgruppen.

Mortalität nach Durchführung der PCPA im Vergleich zwischen den drei Altersgruppen

$$
\text { „frühzeitig“ „elektiv“ „spät“ }
$$

$\begin{array}{lll}\text { Tod insgesamt } & 2(5,3 \%) \quad 16(12,6 \%) & 2(5,3 \%)\end{array}$

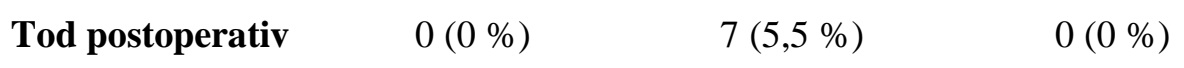

Tod spät $\quad 2(5,4 \%) \quad 9(7,1 \%) \quad 2(5,3 \%)$

Tabelle 4: Ausschnitt aus der Überlebensstatistik nach Durchführung der partiellen cavopulmonalen Anastomose (PCPA) bei einer medianen Beobachtungszeit von 2 Jahren bezogen auf die drei Altersgruppen: Als "frühzeitig“" wird ein Alter bei PCPA von kleiner gleich 90 Tagen (d), als „elektiv“ zwischen 91 und 182 d und als "spät" von über $182 \mathrm{~d}$ bezeichnet. „Tod insgesamt“ bezeichnet den Eintritt des Todes bis zum Ende des

Beobachtungszeitraums, „Tod postoperativ“ den Eintritt des Todes innerhalb von $30 \mathrm{~d}$ nach Durchführung der PCPA, „Tod spät“ den Eintritt des Todes zwischen $31 \mathrm{~d}$ nach Durchführung der PCPA und dem Ende des Beobachtungszeitraums. Absolute Anzahl und Anteil an der Gruppe in Klammern. Es konnten keine signifikanten Unterschiede zwischen den Altersgruppen ermittelt werden. Das Signifikanzniveau lag bei p < 0,05. Anzahl der Patienten pro Messparameter jeweils für die „frühzeitige“, dir „elektive“ und die „späte“ Gruppe: Tod insgesamt n $=38, \mathrm{n}=127$ und $\mathrm{n}=38$; Tod postoperativ $\mathrm{n}=37, \mathrm{n}=127$ und $\mathrm{n}=38$; Tod $\operatorname{spät} \mathrm{n}=37, \mathrm{n}=126$ und $\mathrm{n}=38$. 


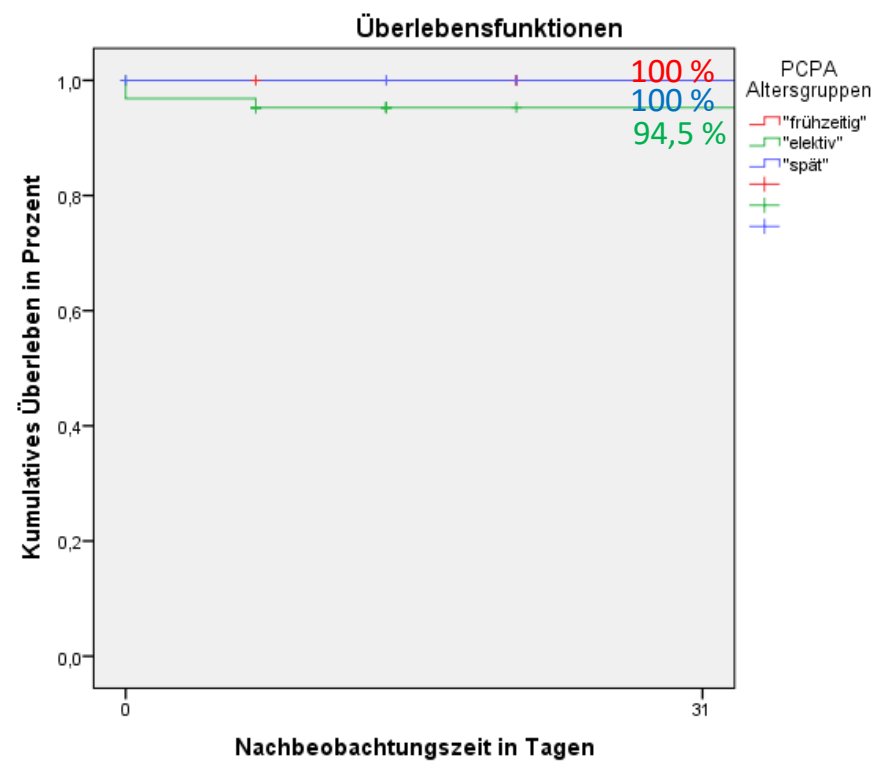

Abbildung 26: Vergleich des 30-Tages-Überlebens nach PCPA zwischen den drei Altersgruppen

Kaplan-Meier-Kurve zum kumulativen Überleben innerhalb der ersten 30 Tage (d) nach Durchführung der partiellen cavopulmonalen Anastomose (PCPA) bezogen auf die drei Altersgruppen: Als „frühzeitig“"wird ein Alter bei PCPA von kleiner gleich 90 Tagen (d) (rot), als „elektiv“ zwischen 91 und 182 d (grün) und als "spät“ von über $182 \mathrm{~d}$ (blau) bezeichnet. Anteil der Überlebenden an der jeweiligen Altersgruppe in Prozent (\%) hinter der entsprechenden Kurve. Es konnten keine signifikanten Unterschiede zwischen den Altersgruppen ermittelt werden. Das Signifikanzniveau lag bei $\mathrm{p}<0,05$. Anzahl der Patienten in der ,frühzeitigen“ Gruppe $\mathrm{n}=37$, in der „elektiven“ Gruppe n = 127 und in der „späten“ Gruppe $\mathrm{n}=38$.

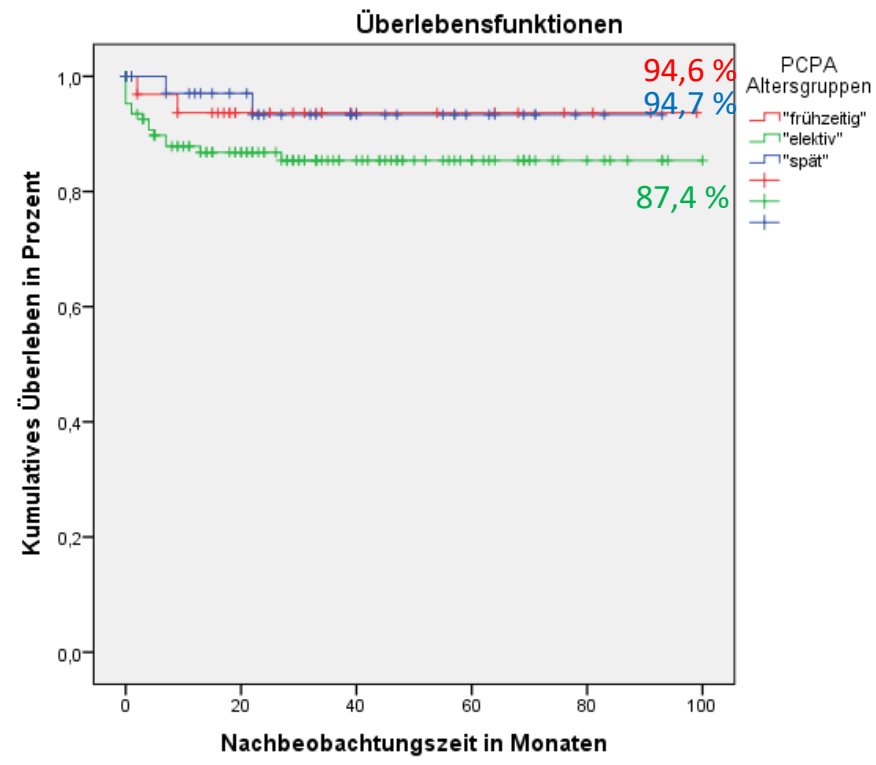

Abbildung 27: Vergleich des Gesamtüberlebens nach PCPA zwischen den drei Altersgruppen

Kaplan-Meier-Kurve zum kumulativen Überleben innerhalb des medianen Nachbeobachtungszeitraumes von zwei Jahren nach Durchführung der partiellen cavopulmonalen Anastomose (PCPA) bezogen auf die drei Altersgruppen Als „frühzeitig“ wird ein Alter bei PCPA von kleiner gleich 90 Tagen (d) (rot), als „elektiv“ zwischen 91 und 182 d (grün) und als „spät“ von über $182 \mathrm{~d}$ (blau) bezeichnet. Anteil der Überlebenden an der jeweiligen Altersgruppe in Prozent (\%) hinter der entsprechenden Kurve. Es konnten keine signifikanten Unterschiede zwischen den Altersgruppen ermittelt werden. Das Signifikanzniveau lag bei $\mathrm{p}<0,05$. Anzahl der Patienten in der „frühzeitigen“ Gruppe n = 37, in der „elektiven“ Gruppe $n=127$ und in der „späten“ Gruppe n $=38$. 


\subsubsection{Einflussfaktoren auf die Mortalität}

In der Cox-Regression stellte sich weder das Alter bei PCPA, noch der Nakata-Index, LPA- oder RPA-Flächen-Index vor PCPA als signifikanter Einflussfaktor auf die Mortalität dar. Das

Körpergewicht zum Zeitpunkt der PCPA erwies sich als signifikanter, negativer Einflussfaktor auf die Mortalität $(p=0,001 ; \operatorname{Exp}(B)=0,999 ;$ Konfidenzintervall $=0,999-1,000)$.

\section{Diskussion}

\subsection{Größe und Wachstum der Pulmonalarterien}

Die PCPA stellt einen wichtigen Schritt auf dem Weg zur Fontan-Zirkulation im Rahmen der Palliation des funktionell univentrikulären Herzens dar. Den hämodynamischen Vorteilen, zu denen die frühzeitige Anlage einer PCPA führt, steht die Möglichkeit eines gehemmten Wachstums der Pulmonalarterien durch den Wegfall des pulsatilen Blutflusses entgegen. Ziel dieser Studie war es den Einfluss einer frühzeitigen PCPA auf das Wachstum der Pulmonalarterien zu untersuchen.

Die mittleren Flächen-Indices beider Pulmonalarterien vor PCPA stellten sich in unserer Studie als signifikant kleiner bei den zum Zeitpunkt der PCPA jüngeren Patientengruppen dar. Zu diesem Ergebnis kamen auch Cleuziou et al. als sie die Durchmesser der Pulmonalarterien vor PCPA von Patienten verglichen, die zum Zeitpunkt der PCPA entweder unter oder über sechs Monate alt waren (Cleuziou et al. 2008). Da zu diesem Zeitpunkt die Dauer, in welcher der lungenperfundierende Shunt auf die Pulmonalarterien wirken konnte bei den jüngeren Patienten im Vergleich zu den älteren Patienten verkürzt war, könnte die bereits mehrfach postulierte Rolle des pulsatilen Blutflusses als stimulierender Faktor auf das pulmonale Gefäßwachstum erklärend für dieses Ergebnis sein (Bossers et al. 2016; Penny et al. 1995; Reddy et al. 1996).

So müssten die Pulmonalarterien der zum Zeitpunkt der PCPA jüngeren Patienten also auch vor TCPA deutlich kleiner sein als die Gefäße der älteren Patienten. Bei unseren Messungen vor TCPA konnten wir jedoch nur signifikant kleinere Nakata-Indices und Flächen-Indices der linken Pulmonalarterie bei den „Frühzeitigen“ im Vergleich zu den „Späten“ beobachten. Es konnte also kein signifikanter Unterschied in der Größe der Pulmonalarterien vor TCPA zwischen Patienten, die zum Zeitpunkt der PCPA unter bzw. über drei Monate alt waren, festgestellt werden. $\mathrm{Zu}$ einem vergleichbaren Ergebnis kamen auch Jaquiss et al., als sie die Pulmonalarteriendurchmesser vor der Fontan-Komplettierung von Patienten, die über oder unter vier Monate bei PCPA alt waren, verglichen (Jaquiss et al. 2006).

Unter Einbeziehung der vor der PCPA ermittelten Ergebnisse, kann man also davon ausgehen, dass die Pulmonalarterien der „Frühzeitigen“ sogar stärker gewachsen sind als die der „Elektiven“.

Während einige Studien von einem Rückgang der Nakata-Indices nach PCPA berichteten, konnten wir sogar eine signifikante Zunahme der Nakata-Indices bei Patienten, die zum Zeitpunkt der PCPA jünger als drei Monate alt waren, beobachten (Kansy et al. 2013; Penny et al. 1995). Auch die mittleren RPA-Flächen-Indices waren in den beiden jüngeren Altersgruppen vor TCPA signifikant größer als vor PCPA, was ein stattgefundenes Gefäßwachstum zwischen beiden Operationen zeigt.

Außerdem unterschieden sich die vor PCPA und die vor TCPA gemessenen mittleren Flächen-Indices der linken Pulmonalarterie der „Frühzeitigen“ nicht, während die Werte der beiden anderen Altersgruppen vor TCPA sogar signifikant kleiner waren als vor PCPA. Diese Abnahme ergibt sich aus der Formel zur Berechnung des Flächen-Index. Die Fläche der linken Pulmonalarterie im Zähler hat nicht so stark zugenommen wie die Körperoberfläche im Nenner. Dadurch wird dieser nur gering 
gestiegene Wert durch eine größere Zahl dividiert und der Wert des Index wird kleiner. Dieses Ergebnis zeigt, dass die linke Pulmonalarterie in keiner der drei Altersgruppen ein zum somatischen Wachstum des Patienten angemessenes Wachstum zwischen den beiden Operationen zeigte.

Eine mögliche Erklärung für dieses Ergebnis könnten die beengten anatomischen Verhältnisse für die linke Pulmonalarterie sein, die durch die Anlage der Damus-Kaye-Stansel-Anastomose und den Aufbau des Aortenbogens im Rahmen der Norwood-Operation entstehen. Dieses Verfahren wird insbesondere bei Patienten mit HLHS, aber auch bei allen Patienten mit funktionell univentrikulärem Herzen und zu kleiner Aortenklappe und/oder Aorta ascendens und/oder relevanter linksventrikulärer Ausflussbahnobstruktion durchgeführt und besteht aus der Anastomose des Pulmonalarterienstammes mit der hypoplastischen Aorta ascendens. Wir konnten zeigen, dass Patienten mit HLHS signifikant kleinere LPA-Flächen-Indices vor PCPA und vor TCPA aufwiesen als Patienten mit anderen Herzfehlern. Bei einem Anteil des HLHS von 41,5\% am gesamten Patientenkollektiv, könnten die anatomischen Verhältnisse nach der Norwood-Operation eine Erklärung für die niedrigeren Werte der LPA-Flächen-Indices sein. Diese Theorie führten auch andere Studien mit ähnlichen Ergebnissen an, wobei der Anteil der Patienten mit HLHS bei $80 \%$ bzw. $20 \%$ am gesamten Patientenkollektiv lag (Cleuziou et al. 2008; Jaquiss et al. 2006).

Eine andere Hypothese wäre ein möglicher Zusammenhang zwischen einer Stenose der linken Pulmonalarterie vor PCPA und einem damit verminderten Wachstum dieses Gefäßes aufgrund eines durch die Stenose bedingten geringeren Blutflusses. Wir konnten nachweisen, dass Patienten mit einer Stenose der linken Pulmonalarterie vor PCPA sowohl kleinere mittlere LPA-Flächen-Indices vor PCPA als auch vor TCPA zeigten, als Patienten ohne diese Stenose. Der Anteil der Patienten mit einer Stenose der linken Pulmonalarterie vor PCPA in unserem Patientenkollektiv lag bei $22,1 \%$ und unterschied sich zwischen den Altersgruppen nicht signifikant.

Eine weitere Erklärung für das unzureichende Wachstum der linken Pulmonalarterie könnte die Lage der PCPA selbst sein. In mehreren Studien wurde beobachtet, dass die zur PCPA eher kontralateral gelegene linke Pulmonalarterie im Vergleich zur ipsilateral gelegenen rechten Pulmonalarterie ein geringeres Wachstum zwischen PCPA und TCPA zeigte und somit die Lage der PCPA in Bezug auf die Pulmonalarterien ebenfalls einen Einfluss auf das Wachstum dieser Gefäße haben könnte (Jaquiss et al. 2006; Mendelsohn et al. 1994; Slavik et al. 1995).

Auch die Lage des lungenperfundierenden Shunts könnte in diesem Zusammenhang von Bedeutung sein. Mehrere Studien führten an, dass bei Vorliegen eines mBT-Shunts der nähere Bezug dieses Shunts zur rechten Pulmonalarterie für die geringere Entwicklung der linken Pulmonalarterie verantwortlich sein könnte (Batra et al. 2005; Caspi et al. 2008; Cleuziou et al. 2008; Jaquiss et al. 2006).

Andere Studien beobachteten hingegen ein gleichmäßiges Wachstum beider Pulmonalarterien bei Vorliegen eines (m)BT-Shunts (Godart et al. 1998; Jahangiri et al. 1999).

Im Gegensatz dazu konnten wir feststellen, dass Patienten mit SP-Shunt (zAP oder mBT-Shunt) sowohl vor PCPA als auch vor TCPA signifikant größere Nakata-Indices und LPA-Flächen-Indices aufwiesen als Patienten mit RVPA-Shunt. Die Werte der RPA-Flächen-Indices wiesen die selbe Tendenz auf, das Ergebnis stellte sich aber als nicht signifikant heraus.

In mehreren Studien, wie zum Beispiel auch dem Single Ventricle Reconstruction Trial, wurde ebenfalls gezeigt, dass Patienten mit einem mBT-Shunt vor PCPA signifikant größere Pulmonalarterien aufwiesen, als Patienten mit RVPA-Shunt, wobei die Shuntgrößen deren in unserer 
Studie entsprachen. Eine Hypothese für die shuntabhängige Größenentwicklung der Pulmonalarterien wurde dabei nicht aufgestellt (Aiyagari et al. 2013; Ohye et al. 2010).

Eine mögliche Erklärung könnte die Länge des mBT-Shunts im Vergleich zu der des RVPA-Shunts sein. Trotz des kleineren Durchmessers des mBT-Shunt, könnte es durch die geringere Shuntlänge zu einem verminderten Druckabfall und somit zu einem vermehrten Blutfluss kommen, was wiederum zu einem gesteigerten Gefäßwachstum der Pulmonalarterien führen könnte. Obwohl der RVPA-Shunt eine höhere Pulsatilität im Vergleich zum mBT-Shunt aufweist, könnte der höhere Mitteldruck des mBT-Shunts verantwortlich für das bessere Wachstum der Pulmonalarterien sein. Diese Hypothese unterstützen die Ergebnisse einer Studie von Bove et al., die in einem computerbasierten Modell niedrigere mittlere Drücke in den Pulmonalarterien bei Vorliegen eines RVPA-Shunts im Vergleich zum mBT-Shunt nachwiesen (Bove et al. 2008). Auch Mair et al. beobachteten einen niedrigeren pulmonalen Blutfluss bei Vorliegen eines RVPA-Shunts im Vergleich zum BT-Shunt und äußerten das Risiko eines eingeschränkten Wachstums der Pulmonalarterien durch dieses Ergebnis (Mair et al. 2003).

Eine andere Theorie stellten Griselli et al. auf, nachdem sie beobachtet hatten, dass das Vorliegen eines RVPA-Shunts mit einem gehäuften Auftreten einer Stenose der zentralen Pulmonalarterie assoziiert ist. Die mögliche Begründung dieses Zusammenhangs besagt, dass es durch den vorwiegend systolischen Fluss durch den RVPA-Shunt zu Verwirbelungen des Blutstroms kommen könnte, die inflammatorisch bedingte Fibrosierungen und dadurch Stenosierungen verursachen könnten (Griselli et al. 2006). Auch wir konnten ein gehäuftes Auftreten von Shuntstenosen, sowie von Stenosen der linken und/oder beiden Pulmonalarterien bei Vorliegen eines RVPA-Shunts zeigen.

Im Gegensatz dazu berichten einige Studien wiederum über ein verbessertes und ausgeglicheneres Wachstum der Pulmonalarterien bei Vorliegen eines RVPA-Shunts durch die höhere Pulsatilität des Blutflusses sowie einer gleichmäßigeren Verteilung des Blutflusses auf beide Pulmonalarterien (Caspi et al. 2008; Fiore et al. 2011; Mery et al. 2011; Pruetz et al. 2009).

Ein Einfluss der unterschiedlichen chirurgischen Techniken in den einzelnen Zentren könnte in Bezug auf die RVPA-Shuntanlage und das Wachstum der Pulmonalarterien bei den kontroversen Ergebnissen ebenfalls eine Rolle spielen.

\subsection{Postoperativer Verlauf, Mortalität und Komplikationen}

Um die Bedeutung von kleineren Pulmonalarterien zu untersuchen, verglichen wir den postoperativen Verlauf nach PCPA sowie die Mortalität bei einem medianen Beobachtungszeitraum von zwei Jahren zwischen den drei Altersgruppen.

Lediglich in den ersten 24 Stunden nach PCPA zeigten die „Frühzeitigen“ eine signifikant niedrigere mittlere arterielle Sauerstoffsättigung im Vergleich zu den beiden anderen Altersgruppen, die allerdings noch im klinisch akzeptablen Bereich lag.

Diese Ergebnisse bestätigten auch Jaquiss et al., die bei Patienten, die zum Zeitpunkt der PCPA unter vier Monaten alt waren, eine initial niedrigere postoperative Sauerstoffsättigung sowie einen verlängerten Aufenthalt im Krankenhaus und auch auf der Intensivstation im Vergleich zu älteren Patienten feststellen. Die Unterschiede der postoperativen Sauerstoffsättigung waren aber auch in dieser Studie in einem akzeptablen Rahmen (75\% vs. $81 \%)$. Die niedrigere postoperative Sauerstoffsättigung konnte in unserer Studie wie auch bei Jaquiss et al. nicht auf einen erhöhten TPG oder PAP zurückgeführt werden, da sich dieser in den ersten $24 \mathrm{~h}$ postoperativ nicht zwischen den 
Altersgruppen unterschied. Als mögliche Erklärung für die initial niedrigeren Werten der jüngeren Patienten bieten Jaquiss et al. die ,[...] exagerated susceptibility to bypass-induced injury in younger

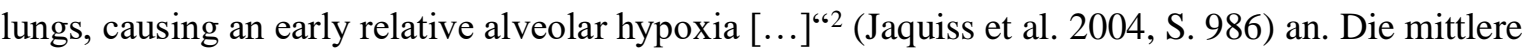
Dauer des kardiopulmonalen Bypasses unterschied sich dabei nicht zwischen den Altersgruppen, allerdings war diese länger als in unserer Studie. Außerdem konnten Jaquiss et al. ebenfalls keine signifikant niedrigeren Werte der mittleren Sauerstoffsättigungen zum Zeitpunkt der Entlassung bei den jüngeren Patienten feststellen (Jaquiss et al. 2004).

Die Hypothese, dass der Einsatz der Herz-Lungen-Maschine für die initial niedrige Sauerstoffsättigung postoperativ zu verantworten sei, führte bereits eine Studiengruppe 1996 an, bei der aufgefallen war, dass Patienten, die ohne den Einsatz eines kardiopulmonalen Bypasses operiert wurden, keine postoperative Hypoxie zeigten (Reddy et al. 1996). Dieser auch bei Erwachsenen beobachtete Zusammenhang wird durch ein multifaktorielles Geschehen erklärt, das sowohl die Entstehung einer systemischen Entzündungsreaktion als auch physiologische Änderungen wie eine Erhöhung des intrapulmonalen Rechts-Links-Shunts und ein vermehrtes Auftreten von Atelektasen umfasst (Asimakopoulos et al. 1999; Weiss et al. 2000). Bei Kindern sind unter anderem ein junges Alter und niedriges Gewicht zum Zeitpunkt der Operation sowie eine Volumen- und/oder Druckbelastung des pulmonalen Gefäßbettes präoperativ als Risikofaktoren für die Entstehung einer Bypass-induzierten Lungenverletzung anzusehen (Cui et al. 2009).

Eine mögliche Erklärung für die initial niedrigeren mittleren arteriellen Sauerstoffsättigungen der „Frühzeitigen“ in unserer Studie könnte sein, dass diese Patienten nicht elektiv, sondern aufgrund eines therapieresistenten, schlechteren klinischen Zustandes frühzeitig operiert wurden. Daher war ihre Lungenfunktion aufgrund von einer shuntbedingten Überflutung des pulmonalen Gefäßbettes mit reaktiver pulmonaler Hypertonie, Wassereinlagerungen sowie dauerhaftem Sauerstoffbedarf bereits präoperativ reduziert im Vergleich zu den Patienten mit einer elektiven oder späten PCPA.

Trotzdem konnten die „Frühzeitigen“" ebenso früh postoperativ extubiert werden, wie die „Elektiven“. Die mediane postoperative Beatmungsdauer unterschied sich in unserer Studie lediglich zwischen den „Frühzeitigen“ und den „Späten“, im Gegensatz zu einer anderen Studie mit gleich alten Patienten (Petrucci et al. 2010). Die Länge des Intensivaufenthaltes unterschied sich ebenfalls nicht signifikant zwischen den beiden jüngeren Gruppen, trotz des reduzierten klinischen Zustandes der „Frühzeitigen“ präoperativ. Des Weiteren zeigte die transkutane Sauerstoffsättigung bei Entlassung zwischen den drei Altersgruppen keine signifikanten Unterschiede, wie von Petrucci et al. ebenfalls beschrieben.

Letztendlich zeigte sich eine signifikant längere Aufenthaltsdauer im Krankenhaus bei den „Frühzeitigen“ im Vergleich zu den „Elektiven“ und den „Späten“, welche sich durch die präoperative Gedeihstörung bei Herzinsuffizienz oder Zyanose in der Gruppe der „Frühzeitigen“ erklären lässt. Petrucci et al. konnten wie wir einen längeren Krankenhausaufenthalt bei den jüngeren Patienten nachweisen. Allerdings gibt es auch eine Studie, die keinen Zusammenhang zwischen dem Alter zum Zeitpunkt der PCPA und der Aufenthaltsdauer im Krankenhaus feststellen konnte, wobei das mediane Alter des Patientenkollektivs bei PCPA bei 5,5 Monaten lag (Kogon et al. 2008).

Bezüglich der postoperativen Mortalität konnten wir keine signifikanten Unterschiede zwischen den drei Altersgruppen feststellen. Die Pulmonalarterien-Indices vor PCPA hatten keinen signifikanten Einfluss auf die Mortalität. Zu vergleichbaren Ergebnissen die Mortalität betreffend, kam eine Studie mit einem medianen Beobachtungszeitraum von 6,6 Jahren hinzu, wobei die Patienten zum Zeitpunkt der PCPA mit im Median 9,6 Monaten deutlich älter und der Anteil der Patienten mit HLHS mit 29\%

\footnotetext{
${ }^{2}$ „erhöhte Anfälligkeit für eine Bypass-induzierte Verletzung in jüngeren Lungen, die eine frühe relative alveoläre Hypoxie verursacht“ (Übersetzung des Verfassers)
} 
am Studienkollektiv deutlich geringer war als in unserer Studie (d'Udekem et al. 2012). Lee et al. zeigten ebenfalls keinen Einfluss des Nakata-Index auf die Mortalität (Lee et al. 2012).

Zusammenfassend lässt sich sagen, dass bei den Patienten in unserer Studie, die eine frühzeitige PCPA erhielten, diese Operation dringend notwendig war um z.B. bei Vorliegen einer frühzeitigen Zyanose eine zusätzliche Shunt-Operation zu vermeiden oder bei Vorhandensein einer Gedeihstörung, Trinkschwäche oder eines anhaltenden Sauerstoffbedarfs die Zeichen einer Herzinsuffizienz positiv zu beeinflussen bzw. günstigere hämodynamische Verhältnisse zu schaffen. Trotz dieser kritischen und interventionsbedürftigen klinischen Zustände präoperativ, konnten wir zeigen, dass diese Patienten keinen komplikationsreicheren postoperativen Verlauf und keinen signifikanten Unterschied in der Mortalität als die elektiv operierten und somit vergleichsweise älteren Patienten aufwiesen.

In Zukunft sollte die Entwicklung der Pulmonalarterien über einen längeren Zeitraum beobachtet werden, um einen möglichen Zusammenhang zwischen einer frühzeitig durchgeführten PCPA bzw. kleineren Pulmonalarterien und dem Auftreten von Langzeitkomplikationen, wie dem enteralen Eiweißverlustsyndrom und der plastischen Bronchitis zu prüfen.

Mehrere Studien zeigten eine Abnahme der Nakata-Indices nach der Fontan-Komplettierung (Adachi et al. 2007) und zum Teil auch einen Zusammenhang zwischen den kleineren Indices und Langzeitkomplikationen (Ovroutski et al. 2009).

Andere Studien liefern hingegen Hinweise darauf, dass kleinere Pulmonalarterien zumindest den kurzund mittelfristigen postoperativen Verlauf nach der Fontan-Komplettierung nicht negativ beeinflussen (Adachi et al. 2007; Lehner et al. 2016). Des Weiteren konnten Bossers et al. mittels MRT Untersuchungen ein zur Körperoberfläche angemessenen Wachstum der Pulmonalarterien nach Fontan-Komplettierung zeigen. Die Größe der Pulmonalarterien von Fontan-Patienten waren sogar vergleichbar mit denen einer gesunden Kontrollgruppe (Bossers et al. 2016).

\subsection{Fazit}

Patienten mit einer frühzeitigen PCPA wiesen sowohl vor PCPA als auch vor TCPA eine kleinere linke Pulmonalarterie auf. Zwischen den beiden Operationen konnte in keiner der drei Altersgruppen ein relevantes Wachstum der linken Pulmonalarterie beobachtet werden. Im Gegensatz dazu zeigt die rechte Pulmonalarterie in dieser Zeitperiode ein kompensatorisches Wachstum, sodass sich deren Größe vor TCPA nicht mehr zwischen den Altersgruppen signifikant unterschied. Trotz des Wegfalls des pulsatilen Blutflusses konnte also ein Wachstum der rechten Pulmonalarterie, insbesondere auch bei Patienten mit einer frühzeitigen PCPA und damit einer verkürzten Einwirkung des pulsatilen Shuntflusses, beobachtet werden.

Trotz signifikant kleinerer Pulmonalarterien zeigten Patienten mit frühzeitiger PCPA im Vergleich zu den älteren Patienten keine relevanten Unterschiede im postoperativen Verlauf oder der postoperativen Mortalität bzw. der Gesamtmortalität, bei einer medianen Nachbeobachtungszeit von zwei Jahren.

Diese Ergebnisse lassen darauf schließen, dass eine frühzeitige PCPA eine mögliche Behandlungsoption für Patienten darstellt, deren kritische klinische Situation eine frühzeitige Intervention nötig macht. 


\subsection{Limitationen}

Die hier vorliegende Studie basiert auf einer retrospektiven Datenerhebung. Somit mussten Patienten aus manchen Untersuchungen ausgeschlossen werden, da die entsprechenden Daten nicht erhoben wurden, wie zum Beispiel einer fehlenden Darstellung der Pulmonalarterien in der präoperativen Angiografie.

Des Weiteren wurden die Pulmonalarterien nur einmalig vermessen. Um hier Fehler zu vermeiden, sollten mehrere Personen unabhängig voneinander die Messungen vornehmen und ein Mittelwert für die weitere statistische Untersuchung verwendet werden.

In mehreren Studien wurde neben oder statt des Nakata-Index der Lower-lobe-Index berechnet, der sich aus der Summe der Fläche der rechten und linken Unterlappenarterie geteilt durch die Körperoberfläche des Patienten zusammensetzt. Durch die Verwendung dieses Index erhofft man sich einen verlässlicheren Wert für die Größe und das Wachstum der Pulmonalarterien, da die Unterlappenarterien nicht von Eingriffen an den Pulmonalarterien wie z.B. einer Patcherweiterung betroffen sind und dadurch das Risiko einer Verfälschung von Daten reduziert werden soll. In unserer Studie wurde auf die Berechnung dieses Index verzichtet, da die Verwendung des Nakata-Index sowie der einzelnen Pulmonalarterien-Indices eine gute Vergleichbarkeit mit der Literatur gewährleistet und die Messung am Abgang der Oberlappenarterie in der Regel jenseits der chirurgischen

Patcherweiterung lag.

Trotz des relativ großen Studienkollektivs von 217 Patienten, befanden sich nur je 40 Patienten in der Gruppe der „Frühzeitigen“ und der „Späten“. Eine gleichmäßigere Größenverteilung über die Altersgruppen zum Vorteil der hier kleinen Gruppen, würde die statistische Validität erhöhen.

Des Weiteren sind bei der geringen Anzahl an Todesfällen während der Studienzeit nur begrenzt Aussagen zu den Einflussfaktoren auf die Überlebenswahrscheinlichkeit der Patienten statistisch belegbar.

\section{Ausblick}

Nach unseren Erkenntnissen scheint die Durchführung einer PCPA im Alter von unter drei Monaten ohne klinisch relevante negative Auswirkungen auf den postoperativen Verlauf oder die Mortalität durchführbar zu sein. Auch im Gesamtüberleben konnten wir bei einer medianen Beobachtungszeit von zwei Jahren keine Unterschiede zwischen den Altersgruppen beobachten.

Diese Ergebnisse tragen dazu bei, dass man Patienten, deren klinischer Zustand eine Intervention nötig macht, mit größerer Sicherheit und weniger Bedenken eine frühzeitige PCPA zukommen lassen kann. Dadurch kann man diese Patienten durch die Verbesserung der hämodynamischen Verhältnisse und der positiven Beeinflussung der Zeichen einer Herzinsuffizienz symptomatisch unterstützen oder ihnen im Falle einer frühzeitigen Zyanose eine weitere Shunt-Operation ersparen.

Außerdem könnten unsere Ergebnisse auch dazu anregen, die Durchführung des Aortenbogenaufbaus und der Anlage der Damus-Kaye-Stansel-Anastomose im Rahmen der Norwood-Operation zu optimieren, um hier für das Wachstum der Pulmonalarterien günstigere anatomische Raumverhältnisse zu schaffen.

Um die weitere Entwicklung des Pulmonalarterien-Wachstums sowie mögliche Auswirkungen der kleineren Pulmonalarterien auf den klinischen Zustand und die Inzidenz von Spätkomplikationen, wie 
dem enteralen Eiweißverlustsyndrom und der plastischen Bronchitis, genauer evaluieren zu können, werden dringend Langzeituntersuchungen benötigt.

\section{Zusammenfassung}

Die PCPA stellte einen wichtige Zwischenschritt auf dem Weg zur Palliation des univentrikulären Ventrikels dar, die sich durch die anschließende totale cavopulmonalen Anastomose im Sinne der Fontan-Zirkulation komplettiert. Durch die bei der PCPA entstehende Trennung des Lungen- vom Körperkreislaufes, besteht kein pulsatiler Blutfluss durch das pulmonale Gefäßbett mehr. Da dieser als möglicher stimulierender Faktor für das Gefäßwachstum betrachtet wird, bestehen Bedenken vor einem gehemmten Wachstum der Pulmonalarterien nach dieser Operation. Eine zum Teil notwendige Durchführung der PCPA vor dem üblichen Alter von drei Monaten, verstärkt diese Bedenken durch den noch früheren Wegfall des pulsatilen Blutflusses.

Das Ziel dieser Arbeit war es, den Einfluss einer frühzeitigen PCPA auf das Wachstum der Pulmonalarterien zu untersuchen. Hierzu wurden 217 Patienten die zwischen 2006 und 2014 eine PCPA am Deutschen Herzzentrum München erhielten, anhand ihres Alters zum Zeitpunkt dieser Operationen in drei Altersgruppen eingeteilt. Die Durchmesser beider Pulmonalarterien wurden retrospektiv ausgemessen und genau wie diverse postoperative Werte zwischen den drei Altersgruppen verglichen. Die Daten hierfür wurden aus Angiografien entnommen, die sowohl kurz vor der partiellen, als auch kurz vor der totalen cavopulmonalen Anastomose an den Patienten standardmäßig durchgeführt wurden.

Es stellte sich heraus, dass Patienten mit einer frühzeitigen PCPA sowohl kurz vor der partiellen als auch kurz vor der totalen cavopulmonalen Anastomose kleinere Pulmonalarterien aufwiesen als das restliche Patientenkollektiv. Dennoch zeigte diese Altersgruppe keinen klinisch relevanten Unterschied im postoperativen Verlauf oder der Mortalität.

Des Weiteren konnte im Gegensatz zur linken Pulmonalarterie ein Wachstum der rechten Pulmonalarterie zwischen PCPA und TCPA im gesamten Patientenkollektiv festgestellt werden. Dies zeigt, dass es trotz des Wegfalls des pulsatilen Blutflusses zu einem Gefäßwachstum kommen kann.

Zusammenfassend lässt sich aus unseren Ergebnissen sagen, dass eine frühzeitige PCPA im Alter von unter drei Monaten eine mögliche Behandlungsmethode für Patienten zu sein scheint, deren klinischer Zustand eine frühzeitige Intervention nötig macht. Die frühere Durchführung der partiellen Kreislauftrennung führt nicht zu einem komplikationsreicheren postoperativen Verlauf oder einer erhöhten Mortalität.

Dennoch sind Langzeitstudien erforderlich, um das Wachstum der Pulmonalarterien, sowie mögliche klinische Konsequenzen kleinerer Pulmonalarterien, wie einer erhöhten Inzidenz von Spätkomplikationen, weiter beobachten zu können. 


\section{Literaturverzeichnis}

1. Adachi, I., Yagihara, T., Kagisaki, K., Hagino, I., Ishizaka, T., Kobayashi, J., Kitamura, S., \& Uemura, H. (2007). Preoperative small pulmonary artery did not affect the midterm results of Fontan operation. European Journal of Cardio-Thoracic Surgery, 32(1), 156-162. doi: https://doi.org/10.1016/j.ejcts.2007.03.024 [09.12.2017]

2. Aiyagari, R., Rhodes, J. F., Shrader, P., Radtke, W. A., Bandisode, V. M., Bergersen, L., Gillespie, M. J., Gray, R. G., Guey, L. T., Hill, K. D., Hirsch, R., Kim, D. W., Lee, K.-J., Pelech, A. N., Ringewald, J., Takao, C., Vincent, J. A., \& Ohye, R. G. (2013). Impact of prestage II hemodynamics and pulmonary artery anatomy on 12-month outcomes in the Pediatric Heart Network Single Ventricle Reconstruction trial. The Journal of Thoracic and Cardiovascular Surgery, 148(4), 1467-1474. doi: https://doi.org/10.1016/j.jtcvs.2013.10.057 [10.12.2017]

3. Asimakopoulos, G., Smith, P. L. C., Ratnatunga, C. P., \& Taylor, K. M. (1999). Lung injury and acute respiratory distress syndrome after cardiopulmonary bypass. The Annals of Thoracic Surgery, 68(3), 1107-1115. doi: https://doi.org/10.1016/S0003-4975(99)00781-X [09.12.2017]

4. Batra, A. S., Starnes, V. A., \& Wells, W. J. (2005). Does the site of insertion of a systemicpulmonary shunt influence growth of the pulmonary arteries? The Annals of Thoracic Surgery, 79(2), 636-640. doi: https://doi.org/10.1016/j.athoracsur.2004.07.062 [09.12.2017]

5. Bossers, S. S. M., Cibis, M., Kapusta, L., Potters, W. V., Snoeren, M. M., Wentzel, J. J., Moelker, A., \& Helbing, W. A. (2016). Long-Term Serial Follow-Up of Pulmonary Artery Size and Wall Shear Stress in Fontan Patients. Pediatric Cardiology, 37(4), 637-645. doi: https://doi.org/10.1007/s00246-015-1326-y [09.12.2017]

6. Bove, E. L., Migliavacca, F., de Leval, M. R., Balossino, R., Pennati, G., Lloyd, T. R., Khambadkone, S., Hsia, T.-Y., \& Dubini, G. (2008). Use of mathematic modeling to compare and predict hemodynamic effects of the modified Blalock-Taussig and the right ventriclepulmonary artery shunts for hypoplastic left heart syndrome. The Journal of Thoracic and Cardiovascular Surgery, 136(2), 312-320. doi: https://doi.org/10.1016/j.jtcvs.2007.04.078 [07.12.2017]

7. Bradley, S. M., Mosca, R. S., Hennein, H. A., Crowley, D. C., Kulik, T. J., \& Bove, E. L. (1996). Bidirectional superior cavopulmonary connection in young infants. Circulation, 94(9 SUPPL), 5-11. Retrieved from https://www-ncbi-nlm-nih-gov.emedien.ub.unimuenchen.de/pubmed/8901711 [10.04.2017]

8. Brown, D. W., Gauvreau, K., Moran, Adrian M. M., Jenkins, K. J. Perry, S. B., del Nido, P. J., \& Colan, S. D. (2003). Clinicial outcomes and utility of cardiac catheterization prior to superior cavopulmonary anastomosis. The Journal of Thoracic and Cardiovascular Surgery, 126(1), 272-281. doi: https://doi.org/10.1016/S0022-5223(03)00054-0 [11.04.2017]

9. Caspi, J., Pettitt, T. W., Mulder, T., \& Stopa, A. (2008). Development of the Pulmonary Arteries After the Norwood Procedure: Comparison Between Blalock-Taussig Shunt and Right Ventricular-Pulmonary Artery Conduit. The Annals of Thoracic Surgery, 86(4), 12991304. doi: https://doi.org/10.1016/j.athoracsur.2008.06.016 [07.12.2017]

10. Chang, A. C., Hanley, F. L., Wernovsky, G., Rosenfeld, H. M., Wessel, D. L., Jonas, R. A., Mayer, J. E., Jr, Lock, J, E., \& Castaneda A. R. (1993). Early Bidirectional Cavopulmonary Shunt in Young Infants. Postoperative Course and Early Results. Circulation, 88(5 part 2), 149-158. Retrieved from http://ovidsp.ovid.com.emedien.ub.unimuenchen.de/ovidweb.cgi?T=JS\&CSC $=$ Y \&NEWS=N\&PAGE=fulltext $\& D=0 v f t \& A N=00003$ 017-199311020-00021\&PDF=y [22.12.2017]

11. Cleuziou, J., Schreiber, C., Cornelsen, J. K., Hörer, J., Eicken, A., \& Lange, R. (2008). BCPC without additional pulmonary blood flow in patients below the age of 6 months. European 
Journal of Cardio-Thoracic Surgery, 34(3), 556-562. doi:

https://doi.org/10.1016/j.ejcts.2008.05.041 [10.12.2017]

12. Cui, Q., Zhou, H., Zhao, R., Liu, J., Zhu, H., Zheng, Q., Gu., C., Yi, D. (2009). The Effects of Open Lung Ventilation on Respiratory Mechanics and Haemodynamics in Atelectatic Infants after Cardiopulmonary Bypass. The Journal of International Medical Research, 37(1), 113120. doi: https://doi.org/10.1177/147323000903700113 [09.12.2017]

13. Douglas, W. I., Goldberg, C. S., Mosca, R. S., Law, I. H., \& Bove, E. L. (1999). Hemi-Fontan Procedure for Hypoplastic Left Heart Syndrome: Outcome and Suitability for Fontan. The Annals of Thoracic Surgery, 68(4), 1361-1367. doi: https://doi.org/10.1016/S00034975(99)00915-7 [10.04.2017]

14. D’Udekem, Y., Xu, M. Y., Galati, J. C., Lu, S., Iyengar, A. J., Konstantinov, I., E., Wheaton, G. R., Ramsay, J. M., Grigg, L. E., Millar, J., Cheung, M. M., \& Brizard, C. P. (2012). Predictors of Survival After Single-Ventricle-Palliation. The Impact of Right Ventricle Dominance. Journal of the American College of Cardiology. 59(13), 1178-1185. doi: https://doi.org/10.1016/j.jacc.2011.11.049 [10.12.2017]

15. Fiore, A. C., Tobin, C., Jureidini, S., Rahimi, M., Kim, E. S., \& Schowengerdt, K. (2011). A Comparison of the Modified Blalock-Taussig Shunt with the Right Ventricle-to-Pulmonary Artery Conduit. The Annals of Thoracic Surgery, 91(5), 1479-1485. doi: https://doi.org/10.1016/j.athoracsur.2010.11.062 [07.12.2017]

16. Ghanayem, N. S., Allen, K. R., Tabbutt, S., Atz, A. M., Clabby, M. L., Cooper, D. S., Eghtesady, P., Frommelt, P. C., Gruber, P. J., Hill, K. D., Kaltman, J. R., Laussen, P. C., Lewis, A. B., Lurito, K. J., Minich, L. L., Ohye, R. G., Schonbeck, J. V., Schwartz, S. M., Singh, R., \& Goldberg, C. S. (2012). Interstage mortality after the Norwood procedure: Results of the multicenter Single Ventricle Reconstruction trial. The Journal of Thoracic and Cardiovascular Surgery, 144(4), 896-906. doi: https://doi.org/10.1016/j.jtcvs.2012.05.020 [10.04.2017]

17. Glenn, W. W. L., Patino, J. F. (1954). Circulatory By-Pass of the Right Reart. I. Preliminary Observations on the Direct Delivery of Vena Caval Blood into the Pulmonary Artery Circulation. Azygos Vein-Pulmonary Artery Shunt. The Yale Journal of Biology and Medicine, 27(3), 147-151. Retrieved from https://www-ncbi-nlm-nih-gov.emedien.ub.unimuenchen.de/pmc/articles/PMC2599414/ [10.04.2016]

18. Godart, F., Qureshi, S. A., Simha, A., Deverall, P. B., Anderson, D. R., Baker, E. J., \& Tynan, M. (1998). Effects of Modified and Classic Blalock-Taussig Shunts on the Pulmonary Arterial Tree. The Annals of Thoracic Surgery, 66(2), 512-517. doi: https://doi.org/10.1016/S00034975(98)00461-5 [07.12.2017]

19. Griselli, M., McGuirk, S. P., Ofoe, V., Stümper, O., Wright J. G. C., de Giovanni, J. V., Barron, D. J., \& Brawn, W. J. (2006). Fate of pulmonary arteries following Norwood Procedure. European Journal of Cardio-Thoracic Surgery, 30(6), 930-935. doi: https://doi.org/10.1016/j.ejcts.2006.08.007 [10.04.2017]

20. Jahangiri, M., Lincoln, C., \& Shinebourne, E. (1999). Does the Modified Blalock-Taussig Shunt Cause Growth of the Contralateral Pulmonary Artery? The Annals of Thoracic Surgery, 67(5), 1397-1399. doi: https://doi.org/10.1016/S0003-4975(99)00144-7 [07.12.2017]

21. Jaquiss, R. D. B., Ghanayem, N. S., Hoffman, G. M., Fedderly, R. T., Cava, J. R., Mussatto, K. A., \& Tweddell, J. S. (2004). Early cavopulmonary anastomosis in very young infants after the Norwood procedure: Impact on oxygenation, resource utilization, and mortality. The Journal of Thoracic and Cardiovascular Surgery, 127(4), 982-989. doi: https://doi.org/10.1016/j.jtcvs.2003.10.035 [10.12.2017]

22. Jaquiss, R. D. B., Siehr, S. L., Ghanayem, N. S., Hoffman, G. M., Fedderly, R. T., Cava, J. R., Mussatto, K. A., \& Tweddell J. S. (2006). Early Cavopulmonary Anastomosis After Norwood 
Procedure Results in Excellent Fontan Outcome. The Annals of Thoracic Surgery, 82(4), 1260-1266. doi: https://doi.org/10.1016/j.athoracsur.2006.04.095 [10.12.2017]

23. Kansy, A., Brzezinska-Rajszys, G., Zubrzycka, M., Mikrowicz-Malek, M., Maruszewski, P., Manowska, M., \& Maruszewski, B. (2013). Pulmonary artery growth in univentricular physiology patients. Kardiologia Polska, 71(6), 581-587. doi: https://doi.org/10.5603/KP.2013.0121 [07.12.2017]

24. Knott-Craig, C. J., Julsrud, P. R., Schaff, H. V., Puga, F. J., \& Danielson G. K. (1993). Pulmonary artery size and clinical outcome after modified Fontan operation. The Annals of Thoracic Surgery, 55(3), 646-651. doi: https://doi.org/10.1016/0003-4975(93)90268-M [11.04.2017]

25. Kogon, B. E., Plattner, C., Leong, T., Simsic, J., Kirshbom, P. M., \& Kanter, K. R. (2008). The bidirectional Glenn operation: A risk factor analysis for morbidity and mortality. The Journal of Thoracic and Cardiovascular Surgery, 136(5), 1237-1242. doi: https://doi.org/10.1016/j.jtcvs.2008.05.017 [09.12.2017]

26. Lee, T. M., Aiyagari, R., Hirsch, J. C., Ohye, R. G., Bove, E. L., \& Devaney, E. J. (2012). Risk Factor Analysis for Second-Stage Palliation of Single Ventricle Anatomy. The Annals of Thoracic Surgery, 93(2), 614-619. doi: https://doi.org/10.1016/j.athoracsur.2011.10.012 [09.12.2017]

27. Lehner, A., Schuh, A., Herrmann, F. E. M., Riester, M., Pallivathukal, S., Dalla-Pozza, R., Kozlik-Feldmann, R., Netz, H., Malec, E., \& Januszewska, K. (2016). Influence of Pulmonary Artery Size on Early Outcome After the Fontan Operation. The Annals of Thoracic Surgery, 97(4), 1387-1393. doi: https://doi.org/10.1016/j.athoracsur.2013.11.068 [09.12.2017]

28. Mair, R., Tulzer, G., Sames, E., Gitter, R., Lechner, E., Steiner, J., Hofer, A., Geiselseder, G., \& Gross, C. (2003). Right ventricular to pulmonary artery conduit instead of modified Blalock-Taussig shunt improves postoperative hemodynamics in newborn after the Norwood operation. The Journal of Thoracic and Cardiovascular Surgery, 126(5), 1378-1384. doi: https://doi.org/10.1016/S0022-5223(03)00389-1 [07.12.2017]

29. Mendelsohn, A. M., Bove, E. L., Lupinetti, F. M., Crowley, D. C., Lloyd, T. R., \& Beekman, R. H. III. (1994). Central pulmonary artery growth patterns after the bidirectional Glenn procedure. The Journal of Thoracic and Cardiovascular Surgery, 107(5), 1284-1290. Retrieved from http://www.jtcvsonline.org/article/S0022-5223(94)70049-4/pdf [07.12.2017]

30. Mery, C. M., LaPar, D. J., Seckeler, M. D., Chamberlain, R. S., Gangemi, J. J., Kron, I. L., \& Peeler, B. B. (2011). Pulmonary Artery and Conduit Reintervention Rates After Norwood Using a Right Ventricle to Pulmonary Artery Conduit. The Annals of Thoracic Surgery, 92(4), 1483-1489. doi: https://doi.org/10.1016/j.athoracsur.2011.04.120 [07.12.2017]

31. Nakanishi, T. (2005). Cardiac Catheterization Is Necessary Before Bidirectional Glenn and Fontan Procedures in Single Ventricle Physiology. Pediatric Cardiology, 26(2), 159-161. doi: https://doi.org/10.1007/s00246-004-0955-3 [11.04.2017]

32. Nakata, S., Imai, Y., Takanashi, Y., Kurosawa, H., Tezuka, K., Nakazawa, M., Ando, M., \& Takao, A. (1984). A new method for the quantitative standardization of cross-sectional areas of the PA in congenital heart diseases with decreased pulmonary blood flow. The Journal of Thoracic and Cardiovascular Surgery, 88(4), 610-619. Retrieved from https://www-ncbi-nlmnih-gov.emedien.ub.uni-

muenchen.de/pubmed/?term $=\mathrm{A}+$ new + method + for + the+quantitative+standardization+of + cross

sectional+areas+of+the+PA+in+congenital+heart+diseases+with+decreased+pulmonary+bloo $\mathrm{d}+$ flow [13.04.2017]

33. Nicolas, R. T., Hills, C., Moller, J. H., Huddleston, C. B., \& Johnson, M. C. (2005). Early Outcome After Glenn Shunt and Fontan Palliation and the Impact of Operating During Viral 
Respiratory Season: Analysis of a 19-Year Multi-Institutional Experience. The Annals of Thoracic Surgery, 79(2), 613-617. doi: https://doi.org/10.1016/j.athoracsur.2004.07.004 [09.12.2017]

34. Ohye, R. G., Devaney, E. J., Hirsch, J. C., \& Bove, E. L. (2007). The Modified BlalockTaussig Shunt Versus the Right Ventricle-to-Pulmonary Artery Conduit for the Norwood Procedure. Pediatric Cardiology, 28(2), 122-125. doi: https://doi.org/10.1007/s00246-0061449-2 [07.12.2017]

35. Ohye, R. G., Sleeper, L. A., Mahony, L., Newburger, J. W., Pearson, G. D., Lu, M., Goldberg, C. S., Tabbutt, S., Frommelt, P. C., Ghanayem, N. S., Laussen, P. C., Rhodes, J. F., Lewis, A. B., Mital, S., Ravishankar, C., Williams, I. A., Dunbar-Masterson, C., Atz, A. M., Colan, S., Minich, L., Pizarro, C., Kanter, K. R., Jaggers, J., Jacobs, J. P., Dent Krawczeski, C., Pike, N., McCrindle, B. W., Vrizi, L., \& Gaynor, W. J. (2010). Comparison of Shunt Types in The Norwood Procedure for Single-Ventricle Lesions. The New England Journal of Medicine, 362(21), 1980-1992. doi: https://doi.org/10.1056/NEJMoa0912461 [07.12.2017]

36. Ohye, R. G., Schonbeck, J. V., Eghtesady, P., Laussen, P. C., Pizarro, C., Shrader, P., Frank, D. U., Graham, E. M., Hill, K. D., Jacobs, J. P., Kanter, K. R., Kirsh, J. A., Lambert, L. M., Lewis, A. B., Ravishankar, C., Tweddell, J. S., Williams, I. A., \& Pearson, G. D. (2012). Cause, timing and location of death in the Single Ventricle Reconstruction trial. The Journal of Thoracic and Cardiovascular Surgery, 144(4), 907-914. doi: https://doi.org/10.1016/j.jtcvs.2012.04.028 [07.12.2017]

37. Ovroutski, S., Ewert, P., Alexi-Meskishvili, V., Hölscher, K., Miera, O., Peters, B., Hetzer, R., \& Berger, F. (2009). Absence of Pulmonary Artery Growth after Fontan Operation and Its Possible Impact on Late Outcome. The Annals of Thoracic Surgery, 87(3), 826-831. doi: https://doi.org/10.1016/j.athoracsur.2008.10.075 [22.12.2017]

38. Penny, D. J., Pawade, A., Wilkinson, J. L., \& Karl, T. R. (1995). Pulmonary Artery Size After Bidirectional Cavopulmonary Connection. Journal of Cardiac Surgery, 10(1), 21-26. doi: https://doi.org/10.1111/j.1540-8191.1995.tb00585.x [10.12.2017]

39. Petrucci, O., Khoury, P., Manning, P. B., \& Eghtesady, P. (2010). Outcomes of the bidirectional Glenn procedure in patients less than 3 months of age. The Journal of Thoracic and Cardiovascular Surgery, 139(3), 562-568. doi: https://doi.org/10.1016/j.jtcvs.2009.08.025 [09.12.2017]

40. Pruetz, J. D., Badran, S., Dorey, F., Starnes, V. A., \& Lewis, A. B. (2009). Differential branch pulmonary artery growth after the Norwood procedure with right ventricle-pulmonary artery conduit versus modified Blalock-Taussig shunt in hypoplastic left heart syndrome. The Journal of Thoracic and Cardiovascular Surgery, 137(6), 1324-1348. doi: https://doi.org/10.1016/j.jtcvs.2009.03.019 [07.12.2017]

41. Reddy, M. V., Liddicoat, J. R., \& Hanley, F. L. (1995). Primary Bidirectional Superior Cavopulmonary Shunt in Infants Between 1 and 4 Months of Age. The Annals of Thoracic Surgery, 59(5), 1120-1126. doi: https://doi.org/10.1016/0003-4975(95)00014-C [09.12.2017]

42. Reddy, M. V., McElhinney, D., B., Moore, P., Hass, G. S., \& Hanley, F. L. (1997). Outcomes After Bidirectional Cavopulmonary Shunt in Infants Less Than 6 Months Old. Journal of the American College of Cardiology, 29(6), 1365-1370. doi: https://doi.org/10.1016/S07351097(97)00068-5 [09.12.2017]

43. Reddy, M. V., McElhinney, D. B., Moore, P., Petrossian, E., \& Hanley, F. L. (1996). Pulmonary artery growth after bidirectional cavopulmonary Shunt: Is there a cause for concern? The Journal of Thoracic and Cardiovascular Surgery, 112(5), 1180-1192. doi: https://doi.org/10.1016/S0022-5223(96)70131-9. [09.12.2017]

44. Rumball, E. M., McGuirk, S. P., Stümper, O., Laker, S. J., de Giovanni, J. V., Wright, J. G., Barron, D. J., \& Brawn, W. J. (2005). The RVPA-Conduit stimulates better growth of the 
pulmonary arteries in hypolplastic left heart syndrome. European Journal of Cardio-Thoracic Surgery, 27(1), 801-806. doi: https://doi.org/10.1016/j.ejcts.2005.01.061 [23.05.2016]

45. Schmid, C., \& Asfour, B. (2009). Leitfaden Kinderherzchirurgie. doi: https://doiorg.emedien.ub.uni-muenchen.de/10.1007/978-3-7985-1709-7 [17.04.2016]

46. Slavik, Z., Lamb, R. K, Webber, S. A, Devlin, A. M., Keeton, B. R., Monro, J. L., \& Salmon, A. P. (1996). Bidirectional superior cavopulmonary anastomosis: how young is too young? Heart, 75(1), 78-82. doi: http://dx.doi.org/10.1136/hrt.75.1.78 [11.04.2017]

47. Slavik, Z., Webber, S. A., Lamb, R. K., Horvath, P., LeBlanc, J. G., Keeton, B. R., Monro, J. L., Tax, P., Tuma, S., Reich, O., Sandor, G. G. S., Daubenes, P. E. F., \& Salmon, A. P. (1995). Influence of Bidirectional Superior Cavopulmonary Anastomosis on Pulmonary Arterial Growth. The American Journal of Cardiology, 76(14), 1085-1087. doi: https://doi.org/10.1016/S0002-9149(99)80307-5 [09.12.2017]

48. Weiss, Y. G., Merin, G., Koganov, E., Ribo, A., Oppenheim-Eden, A., Medalion, B., Peruanski, M., Reider, E., Bar-Ziv, J., Hanson, W. C., \& Pizov, R. (2000).

Postcardiopulmonary Bypass Hypoxemia: Prospective Study on Incidence, Risk Factors, and Clinical Significance. The Journal of Cardiothoracic and Vascular Anesthesia, 14(5), 506513. doi: https://doi.org/10.1053/jcan.2000.9488 [10.12.2017]

49. Zahr, R. A., Kirshbom, P. M., Kopf, G. S., Sainathan, S., Steele, M. M., Elder, R. W., \& Karimi, M. (2016). Half a Century's Experience With the Superior Cavopulmonary (Classic Glenn) Shunt. The Annals of Thoracic Surgery, 101(1), 177-182. doi: https://doi.org/10.1016/j.athoracsur.2015.08.018 [09.12.2017]

50. Ziemer, G., \& Haverich, A. (2010). Herzchirurgie: Die Eingriffe am Herzen und den herznahen Gefäßen. Retrieved from https://books.google.de/books?id=x68kBAAAQBAJ\&lpg=PP1\&hl=de\&pg=PA881\#v=onepa ge\&q\&f=false [02.04.2016] 


\section{Danksagung}

Mein erster Dank gilt Prof. Dr. Peter Ewert, Direktor der Klinik für Kinderkardiologie und angeborene Herzfehler des Deutschen Herzzentrum München, der es mir in seiner Funktion als Doktorvater überhaupt erst ermöglicht hat, mein Promotionsvorhaben durchzuführen und für dessen Unterstützung ich sehr dankbar bin.

Besonders bedanken möchte ich mich außerdem bei Dr. Bettina Ruf, die ihre Rolle als Betreuerin nicht besser hätte erfüllen können. Von Beginn an hat sie mich mit ihrer unermüdlichen Positivität und Herzlichkeit motiviert und unterstützt. Ihr immer offenen Ohr, ihre nicht endende Geduld und ihre stets hilfreichen Ratschläge haben mich bis zur Fertigstellung dieser Dissertation begleitet.

Außerdem möchte ich Dr. Michael Lauseker vom Institut für medizinische Informationsverarbeitung, Biometrie und Epidemiologie meiner Heimatuniversität Ludwig-Maximilian-Universität München für die sehr freundliche und hilfreiche Beratung bezüglich der statistischen Methoden danken.

Zuletzt bedanke ich mich bei meiner Familie und meinen Freunden, die in den letzten Jahren immer eine Stütze für mich waren und denen ich für den immerwährenden Rückhalt vom Herzen danken möchte. 